\title{
Qigong among older adults: a global review
}

\author{
XinQi Dong* and Stephanie Bergren \\ Chinese Health, Aging and Policy Program, Rush Institute for Healthy Aging, Rush University Medical Center, Chicago, IL 60612, USA
}

\begin{abstract}
The review focuses on the state of global Qigong research regarding its prevalence and practice, practitioner characteristics, and its effects on health among older adults. Qigong is a Chinese traditional practice that has been developed for thousands of years for the purposes of improving and sustaining health, and its ease of practice has appealed to older adults and those who wish to find non-biomedical ways to address their health concerns. It is practiced throughout the world, and its practitioners report better health than their non-practitioner counterparts according to cross-sectional, longitudinal, and retrospective studies. Trial research has also shown that Qigong is effective at improving many health outcomes, especially in regard to chronic physical and psychological conditions. Many research gaps exist, especially concerning study design and any investigation into the role of culture in Qigong practice. Future research should closely examine the feasibility and adaptability of Qigong exercise, health outcomes, and exercise adherence for individuals in advancing age, while also evaluating the effects of Qigong versus other forms of mind-body exercise and whether cultural specificity and CAM beliefs affect health outcomes. Last, researchers, health providers, and community leaders should investigate and improve the physical and psychosocial health and health behaviors of older adults through culturally appropriate and adaptable exercises like Qigong.
\end{abstract}

\section{Introduction}

Qigong is a traditional Chinese medicinal practice which is believed to help channel energy $(\mathrm{Q} i)$ to various parts of the body in order to promote health [1]. Its practice has been developed over thousands of years in China and includes many different forms which may be tailored toward addressing specific ailments ranging from cancer to arthritis to poor immune function [2]. In general, Qigong practice includes specific body movements, breathing patterns, and meditation and can be practiced both internally, by the individual, or externally, by a trained Qigong master [3]. There is great variety in Qigong forms, and it can be performed sitting or standing, typically with an emphasis on either movement or meditation [2]. The overarching goal of any Q $i$ energy practice, which also includes Tai Chi, acupuncture, reiki, and martial arts, is to nurture and maintain balance within the body as a means to good health. Thus, Qigong is commonly included in the larger category of "mind-body" techniques.

Qigong has been a large part of Chinese history and health practices, but its appeal and scope extends beyond the confines of traditional Chinese culture. In the mid-twentieth century, Qigong became known as zuguo yixue yichan, (中國醫學遺產) or a "national medical heritage," which formally recognized Qigong in the Chinese state's medical system, largely due to its traditional origins in Daoist practices [4]. Qigong has transitioned from relegation in the traditional Chinese medicine sphere to a broader application including scientific inquiry as a result of globalization and a changing political climate [4] and the global application and practice of Qigong in non-Chinese spaces. In addition to its continued and popular practice in China [5,6], Qigong is practiced around the world, in countries such as the U.S. [7], Taiwan [8], Singapore [9], Canada [10], and Sweden [11], which points to its global relevance as a medical practice. There is some indication that traditional Chinese medicine practices can change significantly once adopted by non-Chinese individuals or those who endorse western medical practices [12]. Therefore, the globalization and modernization of Qigong indicates there may be many more permutations of Qigong than are indicated in historical Chinese record.
An individual's culture and beliefs are an important component of all medical care, whether or not it is classified as traditional, biomedical, psychological, or anywhere in-between. Research in the U.S. has found that personal beliefs are correlated with the decision to practice or use Complementary and Alternative Medicine (CAM), regardless of ethnicity [13]. In medically pluralistic countries, biomedicine may take a prevailing position, but mind-body practices like Qigong remain popular due to cultural ideas of health which cannot be addressed in biomedical practices [14]. The inclusion of traditional medicine in our global health research, policies, and practices may help to achieve a more holistic and person-centered healthcare system, especially among under-represented minority populations [15-17].

Qigong warrants greater attention in research, both as a popular health practice and as a possible intervention for older adults. CAM may be particularly appealing to older adults for multiple reasons. According to the World Health Organization, CAM is appealing due to its safety, quality, efficacy, cost, and accessibility [18]. Further, CAM treatments often focus on the comprehensive health and quality of life of an individual [19], which may be helpful when addressing chronic symptoms where there is not cure. In the U.S., it is estimated that $89.7 \%$ of older adults suffer from at least one chronic condition; $35.9 \%$ have five or more chronic conditions [20]. There is some evidence that older adults use CAM for chronic conditions [21], as well as anecdotal evidence that Qigong is popular among older adults for the same reasons [14]. Qigong, in particular, is a CAM modality which is easy to learn [3], can be adapted for many different ongoing conditions,

Correspondence to: XinQi Dong, Chinese Health, Aging and Policy Program, Rush Institute for Healthy Aging, Rush University Medical Center, 1645 W. Jackson Blvd Suite 675, Chicago, IL 60612, USA, Tel: 312-942-3350; E-mail: xinqi_dong@rush.edu

Key words: Qigong, health, review, older adults

Received: January 10, 2016; Accepted: February 25, 2016; Published: February 29,2016 
including arthritis, blood disorders, memory problems, and depression [2], and may reduce health costs and medical visits [10].

The aims of this review are to understand 1) the prevalence of Qigong practice and characteristics of older adult practitioners globally; 2) the health effects of Qigong practices among older adults; and 3) research gaps in and implications of the practice of Qigong and its impact on the health of the global aging population.

\section{Methods}

The authors conducted separate searches of global literature in English and Chinese databases for thoroughness. For English language publications, the search was conducted on Google Scholar. The searched keywords included the following: older adults, qigong, health, Baduanjin, $Q i$ therapy, $Q i$ training, and external $Q i$. In addition, references from 18 most recent review papers on Qigong were included $[2,3,22-36]$, which were selected based on publication after 2005. Exclusion criteria included non-English language, graduate theses, abstracts, publication date before 1995, insufficient data, did not include adults age 60 and over, only physiological data, and any duplicates across review papers. Full methodology for English publications is shown in Figure 1a. For Chinese language publications, the search was conducted using CNKI including the keywords Qigong (氣功), Baduanjing (八段錦) Wuqinxi (五禽戲), Yijinjing (易筋經), and Liuzijue (六字訣). Exclusion criteria included articles published before 2000, non-academic publications or unpublished manuscripts, unrelated content, graduate theses, did not include adults age 60 and over, lack of data or only physiological data. Full methodology for Chinese publications is shown in Figure $1 b$.

\section{Results}

Our search yielded 62 English-language results and 9 Chinese language results for a total of 71 articles, which were then grouped into several categories: prevalence, practitioner characteristics, nonrandomized control trial (RCT) health outcome studies, and RCT health outcomes studies.

\section{Global prevalence and practice of Qigong}

Table 1 introduces the prevalence of Qigong practice globally. Five papers were found. The three papers about Qigong practice prevalence all came from various waves of the National Health Interview Survey (NHIS), which estimates Qigong practice in the past twelve months by adults is $0.3 \%$ [37,38]. According to Barnes et al. [37], this equates to about 500,000 Qigong practitioners and $70 \%$ of those also practice Tai Chi. The 2002 NHIS survey estimates that $0.1 \%$ of male cancer survivors and $0.5 \%$ of female cancer survivors practice Qigong [39]. Last, a 1999 survey of over two thousand older adults in Singapore finds that 3.5\% of those surveyed practice Qigong [9].

\section{Practitioner characteristics associated with Qigong}

Table 2 introduces the socio-demographic and health characteristics of practitioners. Six papers were found with 1,574 Qigong practitioners surveyed. Four out of six studies examine western populations. Regarding sociodemographic characteristics, Komelski et al. [40] examined Tai Chi Qigong practitioners versus non-practitioners and found a non-significant age difference and higher income $(\mathrm{p}<.001)$ and education levels $(\mathrm{p}<.001)$ in a cross-sectional study. Jouper et al. [11] surveyed reasons for practice among 253 Biyun Qigong practitioners in Swedish and found that $48 \%$ were curious, $9 \%$ desired a low-impact activity, $19 \%$ wanted to promote their health, and $24 \%$ wished to recuperate from illness.
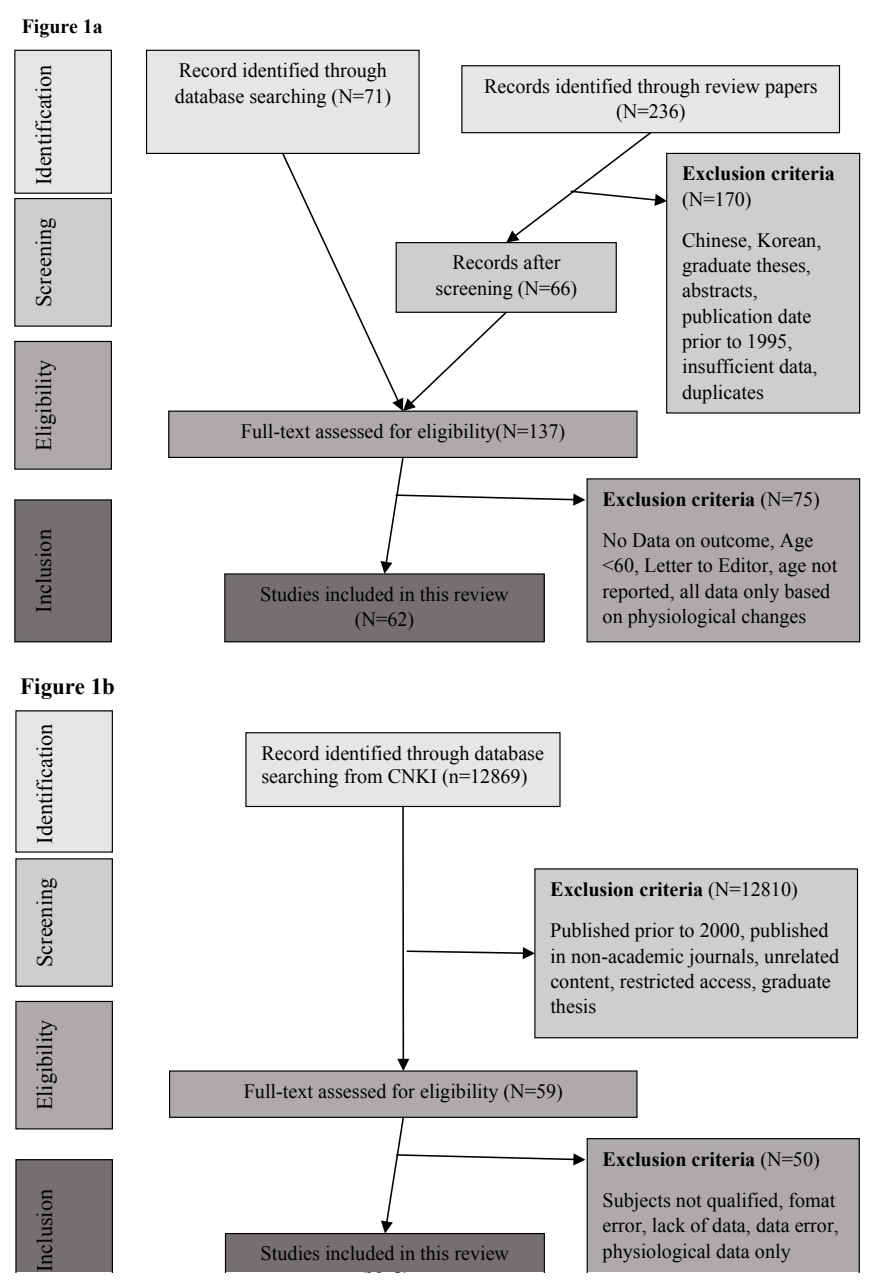

Figure 1:

Qigong practice is positively correlated with self-reported health status and quality of life and has better health status compared to various non-Qigong groups [8,11,40,41]. Ho et al. (2011) [8] found in a cross-sectional survey of 825 individuals that Qigong practitioners in Taiwan are more likely to have better quality of life $(\mathrm{p}<.01)$ and less likely to have diabetes $(\mathrm{p}=.01)$, heart disease $(\mathrm{p}<.001)$, and hypertension $(\mathrm{p}<.001)$ versus non-practitioners. A cross-sectional study in Sweden of Biyun Qigong practitioners found that $52 \%$ of those surveyed continue practice due to better perceived psychological well-being and $24 \%$ continue practice due to better physical health [11]. Similarly, a U.S. cross-sectional study showed Tai Chi Qigong practitioners have significantly better self-reported health compared to a group of moderate exercisers $(\mathrm{p}<.001)$ [40], and a retrospective study in Korea found $40.2 \%$ of nearly 800 participants reported improvement in health after practicing ChunDoSunBup [41]. Additionally, in a study of Qigong meditators versus non-meditators, Qigong practitioners had a significantly lower neuroticism score (mean QG $=7.14 \pm$ 4.45 , mean $\mathrm{NP}=10.15 \pm 4.73, \mathrm{p}<.0001$ ), and years of practice was significantly correlated with lower neuroticism $\left(\mathrm{r}^{2}=0.69, \mathrm{p}<.001\right)$ [42]. Last, a prospective study in Canada also found that Yanxin Qigong 
Table 1. Prevalence and Practice of Qigong by Country.

\begin{tabular}{|c|c|c|c|c|c|}
\hline Author (Year) & Population Setting & Demographics & Survey Method & Survey Response Rate & Prevalence \\
\hline \multicolumn{6}{|l|}{ U.S. } \\
\hline Olano et al. (2015) [38] & $\begin{array}{l}69,149 \text { adults representing } \\
\text { approximately } 170 \text { million } \\
\text { adults }\end{array}$ & $\begin{array}{l}18 \text { and older, } 51 \% \text { Women, } \\
4.4 \% \text { Asian. Mean age: } 45.0 \\
(0.11)\end{array}$ & $\begin{array}{l}2002,2007 \text {, and } 2012 \\
\text { National Health Interview } \\
\text { Survey (NHIS), in person }\end{array}$ & Not reported & $0.3 \%$ practice $\mathrm{QG}$ in the past 12 months \\
\hline $\begin{array}{l}\text { Fouladbakhsh and } \\
\text { Stommel (2010) [39] }\end{array}$ & 2,262 cancer survivors & $\begin{array}{l}\text { Aged } 18 \text { years and older. } \\
1,371 \text { female, mean age } 59.5, \\
\text { and } 891 \text { male, mean age } 65.3\end{array}$ & $\begin{array}{l}\text { Cross-sectional, } 2002 \\
\text { NHIS survey, in person }\end{array}$ & Not reported & $0.1 \%$ men, $0.5 \%$ women practiced QG \\
\hline Birdee et al. (2009) [7] & $\begin{array}{l}31,044 \text { civilian, } \\
\text { noninstitutionalized, household } \\
\text { population adults }\end{array}$ & NR & 2002 NHIS, in person & $74 \%$ & $\begin{array}{l}\text { 500,000 Qigong users in the U.S. } \\
\text { Among QG practitioners, } 70 \% \text { also } \\
\text { reported practicing tai chi. }\end{array}$ \\
\hline Barnes et al. (2008) [37] & $\begin{array}{l}31,044 \text { civilian, } \\
\text { noninstitutionalized household } \\
\text { adults }\end{array}$ & $\begin{array}{l}23,393 \text { adults and 9,417 } \\
\text { children in } 2007\end{array}$ & $\begin{array}{l}2002 \text { and } 2007 \text { NHIS, in } \\
\text { person }\end{array}$ & $\begin{array}{l}\text { 2002: } 74.3 \% \\
\text { 2007: } 67.8 \% \text { (adult), } \\
76.5 \% \text { (child) }\end{array}$ & $0.3 \%$ adults practiced in 2002 and 2007 \\
\hline \multicolumn{6}{|l|}{ Singapore } \\
\hline Lian et al. (1999) [9] & $\begin{array}{l}2494 \text { older adults } 60 \text { years and } \\
\text { older }\end{array}$ & $\begin{array}{l}37.4 \% \text { ages } 60-64 ; 57.8 \% \\
\text { female; } 88.1 \% \text { Chinese and } \\
63.1 \% \text { married }\end{array}$ & Cross-sectional, in person & $88.8 \%$ & $3.5 \%$ practiced $Q G$ \\
\hline
\end{tabular}

QG=Qigong; National Health Interview Survey=NHIS; NR=Not reported

Table 2. Practitioners Characteristics Associated with QG.

\begin{tabular}{|c|c|c|c|c|c|c|c|c|c|c|c|}
\hline \multirow{2}{*}{$\begin{array}{l}\text { Author } \\
\text { (Year) }\end{array}$} & \multirow{2}{*}{$\begin{array}{l}\text { Study } \\
\text { design }\end{array}$} & \multicolumn{6}{|c|}{ Population Characteristics } & \multirow[t]{2}{*}{ Methods } & \multirow{2}{*}{$\begin{array}{l}\text { Outcome of } \\
\text { Interest }\end{array}$} & \multirow[t]{2}{*}{ Measurement } & \multirow[t]{2}{*}{ Critical Findings } \\
\hline & & $\#$ & Country & $\begin{array}{l}\text { Age: mean } \\
\text { (SD), range }\end{array}$ & $\begin{array}{l}\text { Sex: Male, } \\
\text { Female }\end{array}$ & $\begin{array}{l}\text { Previous QG } \\
\text { Practice }\end{array}$ & $\begin{array}{l}\text { Type of } \\
\text { QG }\end{array}$ & & & & \\
\hline $\begin{array}{l}\text { Yan et al. } \\
(2013) \\
{[10]}\end{array}$ & PS & 188 & Canada & $\begin{array}{l}45(11.42) \\
18-82\end{array}$ & $63 \%$ female & $\begin{array}{l}\text { Average } \\
\text { practice } \\
\text { time about } \\
35 \text { months } \\
\text { (Range } 7-81 \\
\text { months) }\end{array}$ & YXQG & $\begin{array}{l}7 \text { year follow } \\
\text { up }\end{array}$ & $\begin{array}{l}\text { Study } \\
\text { relationship } \\
\text { between } \\
\text { medical cost/ } \\
\text { utilization } \\
\text { and YXQG } \\
\text { practice }\end{array}$ & $\begin{array}{l}\text { 1) Monthly medical } \\
\text { visits } \\
\text { 2) Monthly total cost } \\
\text { of medical visits }\end{array}$ & $\begin{array}{l}\text { 1) The average monthly medical } \\
\text { visit for the participants was } 0.97 \\
\text { before practicing YXQG and } 0.71 \\
\text { after practicing YXQG. ( }<.05) \text {. } \\
\text { 2) The average monthly cost of } \\
\text { the sampled individuals before } \\
\text { practicing YXQG was } \$ 41 \text { and } \\
\$ 30 \text { after practicing YXQG. } \\
(\mathrm{p}<.05)\end{array}$ \\
\hline $\begin{array}{l}\text { Komelski } \\
\text { et al. } \\
(2012) \\
{[40]}\end{array}$ & $\mathrm{CS}$ & $\begin{array}{l}120 \mathrm{TQG} \\
414,629 \\
\text { nationally } \\
\text { representative } \\
\text { sample }\end{array}$ & US & $\begin{array}{l}\text { TQG: } 54.78 \\
(10.73), \\
24-83 \\
\text { Comparison: } \\
54.86 \\
(16.74), \\
18-99\end{array}$ & $\begin{array}{l}\text { TQG: } 57 \mathrm{M}, \\
62 \mathrm{~F} \\
\text { Comparison: } \\
155,703 \mathrm{M}, \\
258,806 \mathrm{~F}\end{array}$ & NR & TQG & $\begin{array}{l}\text { Comparison } \\
\text { between TQG, } \\
\text { some exercise, } \\
\text { and no exercise }\end{array}$ & $\begin{array}{l}\text { Examine } \\
\text { and compare } \\
\text { health status } \\
\text { of TQG } \\
\text { practitioners } \\
\text { and nationally } \\
\text { representative } \\
\text { sample }\end{array}$ & $\begin{array}{l}\text { 1) demographics } \\
\text { (age, income, } \\
\text { education) } \\
\text { 2) Self-reported } \\
\text { Health (0-4, poor to } \\
\text { excellent) }\end{array}$ & $\begin{array}{l}\text { 1) NS age difference. } \\
\text { TQG higher income than some } \\
\text { exercise and no exercise group } \\
(5.90 \pm 1.74 \text { vs. } 4.65 \pm 2.15, \mathrm{p}<.001) \\
\text { TQG higher education than some } \\
\text { exercise and no exercise group ( } 4.76 \\
\pm 0.56 \text { vs. } 3.79 \pm 1.09, \mathrm{p}<.001) \\
\text { 2) TQG Exercise group had the } \\
\text { significant highest mean on self- } \\
\text { reported health, followed by the } \\
\text { Some Exercise group and then the } \\
\text { No Exercise group }(3.09 \pm 0.85 \mathrm{vs.} \\
2.62 \pm 1.03 \text { vs. } 1.94 \pm 1.15, \mathrm{p}<.001) \text {. }\end{array}$ \\
\hline $\begin{array}{l}\text { Ho et al. } \\
\text { (2011) [8] }\end{array}$ & $\mathrm{CS}$ & $\begin{array}{l}825 \\
165 \mathrm{WTK} \\
660 \mathrm{NP}\end{array}$ & Taiwan & $\begin{array}{l}\text { QG: 44-90 } \\
\text { NP: matched }\end{array}$ & $\begin{array}{l}41.2 \% \mathrm{M} \\
58.8 \% \mathrm{~F}\end{array}$ & $\begin{array}{l}\text { Over half } \\
\text { a year or } \\
\text { regularly } \\
\text { practiced } \\
\text { at least two } \\
\text { hours per } \\
\text { week for } 26 \\
\text { weeks }\end{array}$ & WTK & $\begin{array}{l}\text { In-person, } \\
\text { cross- } \\
\text { comparison } \\
\text { with NHIS } \\
\text { data of } \\
\text { sedentary } \\
\text { individuals or } \\
\text { other exercise } \\
\text { practitioners }\end{array}$ & $\begin{array}{l}\text { To compare } \\
\text { health } \\
\text { between } \\
\text { WTK } \\
\text { practitioners } \\
\text { and non- } \\
\text { practitioners }\end{array}$ & $\begin{array}{l}\text { 1) Quality of life } \\
\text { (SF-36) } \\
\text { 2) health behaviors } \\
\text { and chronic diseases }\end{array}$ & 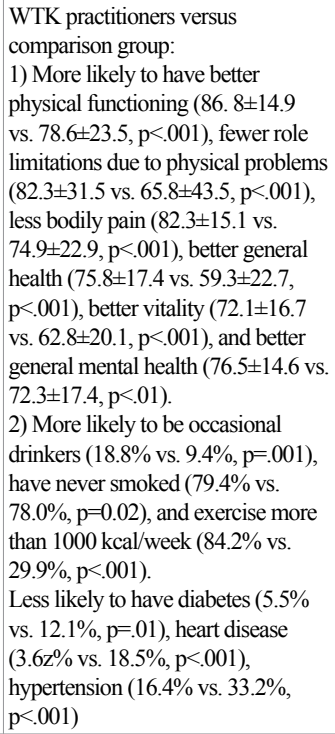 \\
\hline
\end{tabular}




\begin{tabular}{|c|c|c|c|c|c|c|c|c|c|c|c|}
\hline $\begin{array}{l}\text { Jouper } e t \\
\text { al. }(2006) \\
{[11]}\end{array}$ & CS & 253 & Sweden 5 & $58(13)$ & $38 \mathrm{M}, 215 \mathrm{~F}$ & $\begin{array}{l}\text { Recruited } \\
\text { from Qigong } \\
\text { association } \\
77 \\
\text { participants } \\
\text { were } \\
\text { instructors } \\
\text { (Jichugong } \\
35 \%, \\
\text { Donggong } \\
16 \% \text {, Senior } \\
\text { gong } 49 \% \text { ) } \\
\text { Average } \\
\text { completed } \\
4 \pm 4 \text { Qigong } \\
\text { courses }\end{array}$ & $\begin{array}{l}\text { Biyun } \\
\text { method }\end{array}$ & Mail survey & $\begin{array}{l}\text { Describe } \\
\text { how } \\
\text { Swedish } \\
\text { people } \\
\text { practice QG } \\
\text { and reasons } \\
\text { for practice }\end{array}$ & $\begin{array}{l}\text { 1) demographic } \\
\text { characteristics } \\
\text { 2) } \mathrm{QG} \text { practice } \\
\text { behavior } \\
\text { 3) outcomes related } \\
\text { to exercise }\end{array}$ & $\begin{array}{l}\text { 1) Average height } 168(8) \mathrm{cm} \text {, body } \\
\text { mass } 67.5(10) \mathrm{kg} \text {. Lived with partner } \\
(67 \%) \text {, had university degree ( } 57 \%) \text {. } \\
44 \% \text { were employed, } 45 \% \text { retired and } \\
11 \% \text { were students. } \\
\text { 2) Reasons for practice: curiosity } \\
\text { ( } 48 \% \text { ), low-impact activity }(9 \%) \text {, } \\
\text { promote health }(19 \%) \text {, recuperate } \\
\text { from illness }(24 \%) \text {. Practiced Qigong } \\
\text { for average } 5 \text { (SD=3) years with } 4.8 \\
\text { (1.9) sessions per week.65\% prefer } \\
\text { to exercise alone, } 90 \% \text { at home. } 59 \% \\
\text { morning. Qi was perceived as an } \\
\text { internal force by } 47 \% \text {, an emotional } \\
\text { state by } 41 \% \text {, and enhances body } \\
\text { awareness by } 12 \% . \text { All participants } \\
\text { had supplemental physical exercise for } \\
\text { an average of } 49 \text { minutes per day. } \\
3) 52 \% \text { continue practice due to } \\
\text { perceived better psychological } \\
\text { well-being, } 24 \% \text { for physical health } \\
\text { preservation, and } 24 \% \text { to recuperate } \\
\text { from illness. } \\
\text { Health-now was rated by the group } \\
\text { as } 6.9 \pm 1.9 \text { on the } 10 \text {-point scale, and } \\
\text { health-before commencing Qigong } \\
\text { practice as } 4.8 \pm 2.3 \text {, a statistically } \\
\text { significant difference ( }(248)= \\
32.3, \mathrm{p}<05) \text {. Health-now was also } \\
\text { positively correlated with session- } \\
\text { time }(0.17, \mathrm{p}<.01) \text {, years of practice } \\
(0.15, \mathrm{p}<.05) \text { and number of Qigong } \\
\text { courses }(0.14, \mathrm{p}<.05) \text {. } \\
\text { Number of sessions, education, being } \\
\text { an instructor, nor performing other } \\
\text { forms of exercise was correlated to } \\
\text { health-now (all ps }>0.10)\end{array}$ \\
\hline $\begin{array}{l}\text { Leung and } \\
\text { Singhal } \\
(2004) \\
{[42]}\end{array}$ & CS & $\begin{array}{l}154 \\
80 \mathrm{QG} \\
74 \mathrm{NP}\end{array}$ & \begin{tabular}{l|l} 
Canada, & \\
U.S., & 2 \\
Europe & 1 \\
& 2
\end{tabular} & $\begin{array}{l}\text { QG: } 47.5 \text {, } \\
23-69 \\
\text { NP: } 42, \\
28-73\end{array}$ & $\begin{array}{l}\text { QG: } 45 \mathrm{M} \text {, } \\
35 \mathrm{~F} \\
\text { NP: } 29 \mathrm{M} \text {, } \\
45 \mathrm{~F}\end{array}$ & $\begin{array}{l}\text { Mediation: } \\
\text { practice daily } \\
\text { for at least } 12 \\
\text { months } \\
\text { NP: } 20 \\
\text { practiced QG } \\
<12 \text { months }\end{array}$ & $\begin{array}{l}\text { QG } \\
\text { meditation }\end{array}$ & $\begin{array}{l}\text { Online, cross- } \\
\text { compare with } \\
\text { NP }\end{array}$ & $\begin{array}{l}\text { Investigate } \\
\text { if QG } \\
\text { meditation } \\
\text { relates to } \\
\text { personality }\end{array}$ & $\begin{array}{l}\text { 1) Personality: } \\
\text { extraversion and } \\
\text { neuroticism (EPI) }\end{array}$ & $\begin{array}{l}\text { 1) After controlling for age, gender, } \\
\text { and education, years of Qigong } \\
\text { practice and neuroticism negatively } \\
\text { correlated }(\mathrm{r}=-2184, \mathrm{p}<.004) \text {. } \\
\text { Significant difference in neuroticism } \\
\text { between } \mathrm{QG} \text { and } \mathrm{NP} \text { (mean } \mathrm{QG}= \\
7.14 \pm 4.45 \text {, mean } \mathrm{NP}=10.15 \pm 4.73 \text {, } \\
\mathrm{p}<.0001) \text {. } \\
\text { No significant different in extraversion } \\
\text { score between } \mathrm{QG} \text { and } \mathrm{NP} \text { (mean } \\
\mathrm{QG} \text { group }=13.75 \pm 3.05 \text {, mean } \mathrm{NP} \\
=14.04 \pm 3.45, \mathrm{p}>05 \text { ) } \\
\text { Significant strength and predictability } \\
\text { between number of years of } \mathrm{QG} \\
\text { practice and neuroticism }(\mathrm{r}=0.69 \text {, } \\
\mathrm{p}<.001) \text {. }\end{array}$ \\
\hline $\begin{array}{l}\text { Lee } \text { et al. } \\
\text { (2003) } \\
{[41]}\end{array}$ & RS & 768 & Korea & $\begin{array}{l}\text { Ages 16-30 } \\
(31.4 \%), 31- \\
45(33.9 \%), \\
46-60 \\
(21.3 \%), \\
61+(13.4 \%)\end{array}$ & $505 \mathrm{M}, 263 \mathrm{~F}$ & $\begin{array}{l}\text { Practiced } \\
\text { for at least } \\
4 \text { months in } \\
1995\end{array}$ & CDSB & $\begin{array}{l}\text { Ten years } \\
\text { of subjects' } \\
\text { memoranda }\end{array}$ & $\begin{array}{l}\text { Investigate } \\
\text { experience } \\
\text { of medical } \\
\text { conditions } \\
\text { and recovery } \\
\text { after QG } \\
\text { practice }\end{array}$ & $\begin{array}{l}\text { 1) Health issues } \\
\text { 2) Improvement } \\
\text { 3) Motivation } \\
\text { 4) Demographic } \\
\text { Characteristics }\end{array}$ & $\begin{array}{l}\text { 1) Psychological ( } 43.8 \%) \text {, } \\
\text { musculoskeletal (32.4\%), } \\
\text { gastrointestinal ( } 29.9 \%) \\
\text { 2) } 82.8 \% \text { reported improvement in } \\
\text { psychological symptoms, } 76.8 \% \\
\text { improvement in musculoskeletal } \\
\text { symptoms, } 73.3 \% \text { improvement in } \\
\text { gastrointestinal symptoms } \\
66.9 \% \text { reported improvement in } \\
\text { physical health, } 40.2 \% \text { improvement } \\
\text { in overall psychological health } \\
51.8 \% \text { reported marked improvement } \\
\text { in recovery time of wound healing, } \\
66.6 \% \text { reported marked improvement } \\
\text { in inflammation during wound healing } \\
\text { 3) } 81.5 \% \text { reported practicing QG for } \\
\text { health problems } \\
\text { 4) More men }(65.8 \%) \text { then women } \\
\text { (34.2\%), most completed a high } \\
\text { school education (43.8\%) }\end{array}$ \\
\hline
\end{tabular}

$\mathrm{SD}=$ Standard deviation; $\mathrm{QG}=\mathrm{Qigong} ; \mathrm{CS}=$ Cross-sectional study; WTK=Waitankung; NP=Non-practitioners; EPI=Eysenck Personality Inventory; NR=Not reported; TQG=Tai Chi Gong; NS=Not significant; PS=Prospective study; YXQG=Yanxin Qigong; RS=Retrospective study; CDSB=ChunDoSunBup 
practitioners had fewer monthly medical visits and fewer healthcare costs after starting to practice Qigong [10].

\section{Health effects of Qigong in non-RCT studies by primary outcome of interest}

Table 3 presents 13 non-RCT studies from 12 articles which focus on the effects of Qigong on cancer $(n=1)$, diabetes $(n=1)$, musculoskeletal $(n=4)$, physical function $(n=2)$, and psychological $(n=5)$ outcomes.
Three out of ten studies examined the effects of external Qigong therapy (EQT) by a Qigong master, while the remaining seven examined the effects of internal Qigong of various types. The EQT studies have small sample sizes of 13 or fewer individuals [43-45]. Only three studies reported including participants of East Asian decent $[45,46]$. Study design varies widely, ranging from evaluating changes after one 30-minute session of Qigong [47] to a 6-month program of Qigong exercise [48].

Table 3. Non-RCT on QG by Primary Outcome of interest.

\begin{tabular}{|c|c|c|c|c|c|c|c|c|c|c|c|}
\hline \multirow{2}{*}{$\begin{array}{l}\text { Author } \\
\text { (Year) }\end{array}$} & \multirow{2}{*}{$\begin{array}{l}\text { Study } \\
\text { design }\end{array}$} & \multicolumn{6}{|c|}{ Population Characteristics } & \multirow{2}{*}{ Methods } & \multirow[t]{2}{*}{ Outcome of Interest } & \multirow[t]{2}{*}{ Instrument } & \multirow[t]{2}{*}{ Critical Findings } \\
\hline & & $\#$ & Country & $\begin{array}{l}\text { Age: mean } \\
\text { (SD), range }\end{array}$ & Sex & $\begin{array}{l}\text { Previous QG } \\
\text { Practice }\end{array}$ & Type of QG & & & & \\
\hline \multicolumn{12}{|l|}{ Cancer } \\
\hline $\begin{array}{l}\text { Cohen et } \\
\text { al. }(2010) \\
{[45]}\end{array}$ & $\begin{array}{l}\text { Single- } \\
\text { arm pre- } \\
\text { post }\end{array}$ & 9 & $\begin{array}{l}5 \text { US, } 4 \\
\text { China }\end{array}$ & $45-70$ & $9 \mathrm{~F}$ & None & EQT & $\begin{array}{l}5 \text { consecutive days of } \\
\text { EQT, each treatment } \\
2-5 \text { minutes }\end{array}$ & $\begin{array}{l}\text { Test effects of EQT on } \\
\text { breast cancer tumors } \\
\text { and quality of life }\end{array}$ & $\begin{array}{l}\text { 1)Tumor size } \\
\text { US: ultrasound and } \\
\text { mammogram, China: } \\
\text { ultrasound and magnetic } \\
\text { resonance imaging, both: } \\
\text { physical breast examinations } \\
\text { 2) Quality of Life (FACT-G) }\end{array}$ & $\begin{array}{l}\text { 1) No clinically significant change } \\
\text { for all patients (data not shown) } \\
\text { 2) Non-significant changes in } \\
\text { quality of life, pre versus post } \\
(98.3 \pm 13.7 \text { vs. } 94.7 \pm 14.6, p=0.92 \text { ) }\end{array}$ \\
\hline \multicolumn{12}{|l|}{ Diabetes } \\
\hline $\begin{array}{l}\text { Liu et al. } \\
(2010) \\
{[49]}\end{array}$ & OS & 11 & $\mathrm{AU}$ & $42-65$ & $\begin{array}{l}3 \mathrm{M} \\
8 \mathrm{~F}\end{array}$ & NR & $\mathrm{TC} / \mathrm{QG}$ & $\begin{array}{l}\text { Attended } 3 \text { training } \\
\text { classes ( } 60-90 \\
\text { minutes) per week } \\
\text { for } 12 \text { weeks, } \\
\text { received DVD and } \\
\text { were encouraged to } \\
\text { practice at home }\end{array}$ & $\begin{array}{l}\text { Test effects of a TC/QG } \\
\text { exercise program }\end{array}$ & $\begin{array}{l}\text { 1) Hematological } \\
\text { measurements (fasting venous } \\
\text { blood sample) } \\
\text { 2) Quality of life (SF-36) } \\
\text { 3) Physical measures (waist } \\
\text { circumference, BMI, Resting } \\
\text { BP) } \\
\text { 4) Psychological measures } \\
\text { (PSQ; CES-D) }\end{array}$ & 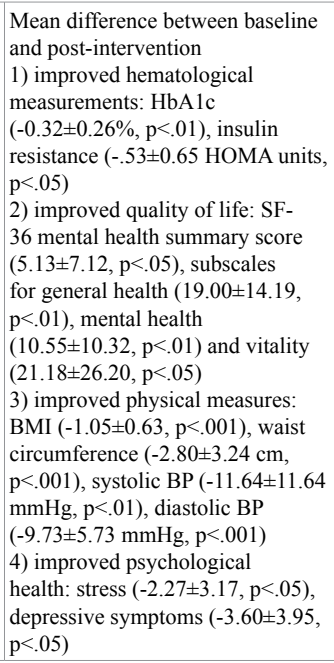 \\
\hline \multicolumn{12}{|c|}{ Musculoskeletal } \\
\hline $\begin{array}{l}\text { Chen } \\
\text { and Liu } \\
(2004) \\
{[43]}\end{array}$ & $\begin{array}{l}\text { Open } \\
\text { trial }\end{array}$ & 10 & US & $58,20-76$ & $\begin{array}{l}3 \mathrm{M} \\
7 \mathrm{~F}\end{array}$ & None & EQT & $\begin{array}{l}3 \text { days, Qigong healer } \\
\text { administers qi for } \\
5-10 \text { min }\end{array}$ & $\begin{array}{l}\text { Test effectiveness of } \\
\text { Qigong on arthritis } \\
\text { symptoms }\end{array}$ & $\begin{array}{l}\text { 1) Pain (VAS) } \\
\text { 2) Mood (VAS) } \\
\text { 3) Pain relief (VAS) } \\
\text { 4) Physical function (ADL) } \\
\text { 5) Anxiety (Spielberger State- } \\
\text { Trait Anxiety) } \\
\text { 6) Swollen/tender joint count } \\
\text { by rheumatologist }\end{array}$ & $\begin{array}{l}6 \text { completed pilot trial } \\
\text { 1) All } 6 \text { reported reduction in VAS } \\
\text { pain (mean reduction=34.7) } \\
\text { 2) } 5 \text { out of } 6 \text { reported reduction } \\
\text { in negative mood (mean } \\
\text { reduction=34.2) } \\
\text { 3) } 5 \text { out of } 6 \text { reported increased } \\
\text { relief, } 1 \text { unchanged (mean } \\
\text { increase }=10.2 \text { ) } \\
\text { 4) Mean decrease in physical } \\
\text { disability scores }=-5.2 \\
5 \text { ) Mean decrease in Anxiety state } \\
\text { score }=-11.8 \\
6 \text { ) } 4 \text { out of } 6 \text { decreased active } \\
\text { pain/tender joint counts. (overall } \\
\text { mean }=-1.5 \text { ) }\end{array}$ \\
\hline $\begin{array}{l}\text { Sawynok } \\
\text { et al. } \\
(2013) \\
{[48]}\end{array}$ & OT & 20 & Canada & $53(9.3)$ & $13 \mathrm{~F}$ & $\begin{array}{l}\text { Completed } \\
\text { level } 1 \\
\text { movement } \\
\text { training }\end{array}$ & CFQ & $\begin{array}{l}\text { Level } 2 \text { meditation } \\
\text { training (two half- } \\
\text { day trainings), } 60 \\
\text { min/day for } 8 \text { weeks } \\
\text { and continued for } 6 \\
\text { months }\end{array}$ & $\begin{array}{l}\text { Test effects of CFQ on } \\
\text { fibromyalgia symptoms }\end{array}$ & $\begin{array}{l}\text { 1) Pain (NRS-PI) } \\
\text { 2) Fibromyalgia impact (FIQ) } \\
\text { 3) Sleep quality (PSQI) } \\
\text { 4) Physical and mental } \\
\text { functions (SF-36) }\end{array}$ & $\begin{array}{l}13 \text { completed and included in } \\
\text { analysis } \\
\text { Outcomes, baseline versus } 6 \\
\text { months: } \\
\text { 1) less pain, mean } 5.7 \pm 1.8 \text { vs. } \\
\text { 3.6 } 6.5, \mathrm{p}=0.012 \\
\text { 2) less impact, mean } 50.9 \pm 16.5 \text { vs. } \\
\text { 29.7 } 17.6, \mathrm{p}=0.036 \\
\text { 3) better sleep quality, mean } \\
\text { 11.5 } 53.6 \text { vs. } 8.5 \pm 4.2, \mathrm{p}=0.004 \\
\text { 4) better physical function: mean } \\
36.2 \pm 9.4 \text { vs. } 42.9 \pm 10.7, \mathrm{p}=0.004 \\
\text { Mental: mean } 41.0 \pm 11.2 \text { vs. } \\
48.1 \pm 9.0, \mathrm{NS}\end{array}$ \\
\hline
\end{tabular}




\begin{tabular}{|c|c|c|c|c|c|c|c|c|c|c|c|}
\hline $\begin{array}{l}\text { Liu et al. } \\
(2012) \\
{[50]}\end{array}$ & $\begin{array}{l}\text { Case } \\
\text { studies }\end{array}$ & 2 & US & $\begin{array}{l}\text { P1) } 55 \\
\text { P2) } 63\end{array}$ & F & NR & NR & $\begin{array}{l}\text { 6-week Qigong } \\
\text { exercise, twice a day }\end{array}$ & $\begin{array}{l}\text { Test effect of QG on } \\
\text { fibromyalgia related } \\
\text { issues }\end{array}$ & $\begin{array}{l}\text { 1) Pain (SF-MPQ) } \\
\text { 2) Fatigue (MFI-20) } \\
\text { 3) Sleep quality (PSQI) } \\
\text { 4) Fibromyalgia Impact (FIQ) } \\
\text { 5) Depression (BDI-II) }\end{array}$ & $\begin{array}{l}\text { Significance not reported } \\
\text { Case 1: } \\
\text { 1) Decreased pain: } 28 \text { to } 12 \\
\text { 2) Decreased fatigue: } 79 \text { to } 58 \\
\text { 3) Better sleep quality: } 17 \text { to } 10 \\
\text { 4) Decreased impact: } 58.8 \text { to } 21.1 \\
\text { 5) Decreased depressive } \\
\text { symptoms: } 17 \text { to } 8 \\
\text { Case 2: } \\
\text { 1) Decreased pain: } 36 \text { to } 22 \\
\text { 2) Decreased fatigue: } 86 \text { to } 70 \\
\text { 3) Better sleep quality: } 12 \text { to } 4 \\
\text { 4) Decreased impact: } 72.0 \text { to } 37.5 \\
\text { 5) Decreased depressive } \\
\text { symptoms: } 28 \text { to } 8\end{array}$ \\
\hline $\begin{array}{l}\text { Chen } e t \\
\text { al. }(2006) \\
{[44]}\end{array}$ & Pilot & 13 & US & $49.8,23-66$ & $13 \mathrm{~F}$ & None & EQT & $\begin{array}{l}\text { 5-7 } 40 \text { to } 45 \text { minute } \\
\text { sessions over } 3 \\
\text { weeks with Qigong } \\
\text { healer, pre and post } \\
\text { assessment and } 3 \\
\text { month follow up }\end{array}$ & $\begin{array}{l}\text { Evaluate EQT in } \\
\text { treating effects of } \\
\text { fibromyalgia }\end{array}$ & $\begin{array}{l}\text { 1) Fibromyalgia impact (FIQ) } \\
\text { 2) Tender point count by } \\
\text { physician evaluation } \\
\text { 3) Depression (BDI-II) } \\
\text { 4) Sleep quality (PSQI) } \\
\text { 5) Anxiety (STAI) } \\
\text { 6) Self-efficacy (LSE) } \\
\text { 7) Pain (MPQ) }\end{array}$ & 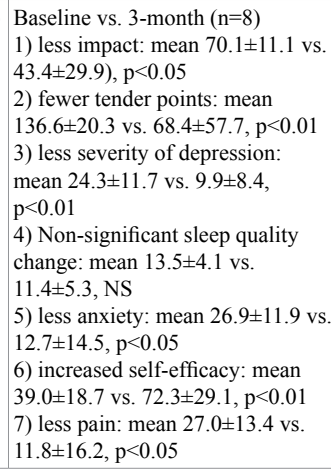 \\
\hline \multicolumn{12}{|c|}{ Physical function } \\
\hline $\begin{array}{l}\text { Sakata } e t \\
\text { al. (2008) } \\
{[46] \mathrm{a}}\end{array}$ & OS & 72 & Japan & $\begin{array}{l}70.9(4.8) \\
60-86\end{array}$ & $72 \mathrm{~F}$ & None & $\begin{array}{l}\text { Floor } \\
\text { 6-style } \\
\text { Qigong } \\
\text { Shaolin } \\
\text { internal } \\
\text { Qigong }\end{array}$ & $\begin{array}{l}12 \text { weeks } \\
90-\text {-min Qigong } \\
\text { exercise program } \\
\text { each week (45-min } \\
\text { Qigong exercise, 20- } \\
\text { min Qigong lecture } \\
\text { and rest, option } \\
\text { 25-min walking in } \\
\text { a swimming pool or } \\
\text { ergometer exercise } \\
\text { 20 min daily Qigong } \\
\text { at home }\end{array}$ & $\begin{array}{l}\text { Determine the effects } \\
\text { of a 12-week Qigong } \\
\text { and aerobic exercise } \\
\text { program on the physical } \\
\text { well-being of relatively } \\
\text { healthy elderly Japanese } \\
\text { women }\end{array}$ & $\begin{array}{l}\text { 1) Physical function: lung } \\
\text { capacity (mL), reaction time } \\
\text { (s), flexibility, walking ability, } \\
\text { balance } \\
\text { 2) Body composition: Bone } \\
\text { mineral content, body fat, soft } \\
\text { tissue lean mass }\end{array}$ & 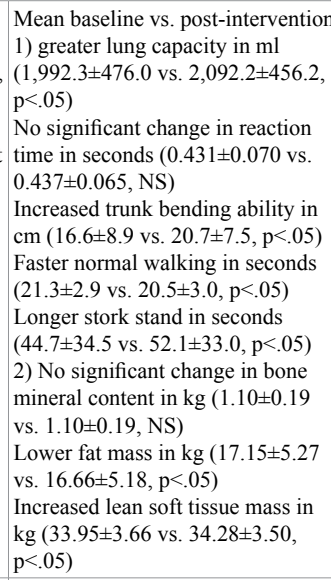 \\
\hline $\begin{array}{l}\text { Sakata } e t \\
\text { al. (2008) } \\
{[46] \mathrm{b}}\end{array}$ & OS & 58 & Japan & $\begin{array}{l}64-71 \\
\text { 1) } 67(1.3) \\
\text { 2) } 67.5(2.0)\end{array}$ & $58 \mathrm{~F}$ & None & $\begin{array}{l}\text { Floor } \\
\text { 6-style } \\
\text { Qigong } \\
\text { Shaolin } \\
\text { internal } \\
\text { Qigong }\end{array}$ & \begin{tabular}{|l}
2 groups: \\
1) Qigong and \\
aerobic exercise \\
(n=29) \\
90-min qigong \\
exercise program \\
each week (45-min \\
Qigong exercise, 20- \\
min Qigong lecture \\
and rest, option \\
25-min walking in \\
a swimming pool or \\
ergometer exercise \\
20 min daily Qigong \\
at home \\
2) Qigong alone \\
(n=29) (walking in \\
swimming pool or \\
ergometer exercise \\
not offered)
\end{tabular} & $\begin{array}{l}\text { Determine the effects } \\
\text { of a 12-week Qigong } \\
\text { and aerobic exercise } \\
\text { program on the physical } \\
\text { well-being of relatively } \\
\text { healthy elderly Japanese } \\
\text { women }\end{array}$ & 1) walking ability, balance & $\begin{array}{l}\text { No significant mean differences } \\
\text { between groups, and both groups } \\
\text { showed significant improvement } \\
\text { in walking and rising between } \\
\text { baseline and post intervention } \\
\text { Group 1: faster walking in seconds } \\
(21.3 \pm 1.9 \text { vs. } 19.6 \pm 1.8, \mathrm{p}<.05) \\
\text { Faster rising from sitting position } \\
\text { in seconds ( } 3.9 \pm 0.8 \text { vs. } 3.2 \pm 0.8 \text {, } \\
\mathrm{p}<.05) \\
\text { Group } 2 \text { : faster walking in seconds } \\
(21.2 \pm 1.9 \text { vs. } 19.7 \pm 1.8, \mathrm{p}<.05) \\
\text { Faster rising from sitting position } \\
\text { in seconds }(3.9 \pm 0.8 \text { vs. } 3.3 \pm 0.9 \text {, } \\
\mathrm{p}<.05)\end{array}$ \\
\hline
\end{tabular}




\begin{tabular}{|c|c|c|c|c|c|c|c|c|c|c|c|}
\hline $\begin{array}{l}\text { Wu et al. } \\
\text { (2014) } \\
{[53] \#}\end{array}$ & Trails & 62 & China & $\begin{array}{l}53(4), \\
41-60\end{array}$ & $\begin{array}{l}28 \mathrm{M}, \\
34 \mathrm{~F}\end{array}$ & none & BDJ & $\begin{array}{l}2 \text { weeks, } 3 \times \text { week, } \\
30 \text { min each } \\
\text { Data collected at } \\
\text { baseline and } 2 \text { weeks }\end{array}$ & $\begin{array}{l}\text { To investigate the effect } \\
\text { of BDJ on the patient } \\
\text { with heart disease and } \\
\text { depression. }\end{array}$ & 1) Depression (SDS, HAMD) & $\begin{array}{l}\begin{array}{l}\text { Between baseline and } 2 \text { weeks, } \\
\text { depressive symptoms improved }\end{array} \\
\text { 1) SDS: } 68 \pm 11.344 \text { vs. } \\
67.63 \pm 11.462, p=.02 \\
\text { HAMD: } 33.23 \pm 6.884 \text { vs. } \\
32.9 \pm 7.139, p=.004\end{array}$ \\
\hline $\begin{array}{l}\text { Johansson } \\
\text { and } \\
\text { Hassmén } \\
(2013) \\
{[47]}\end{array}$ & OS & 43 & Sweden & $59(9.2)$ & $43 \mathrm{~F}$ & $\begin{array}{l}\text { Practicing } \\
\text { for } 7(4.2) \\
\text { years } \\
21 \text { were } \\
\text { Qigong } \\
\text { instructors } \\
\text { and } 25 \text { were } \\
\text { exercisers }\end{array}$ & $\begin{array}{l}\text { Jichu form, } \\
\text { Biyun } \\
\text { qigong } \\
\end{array}$ & $\begin{array}{l}\text { Qigong takes } 30 \\
\text { minutes } \\
\text { Test before activity, } \\
10 \text { minutes into } \\
\text { activity, } 20 \text { minutes } \\
\text { into activity, and post } \\
\text { activity }\end{array}$ & $\begin{array}{l}\text { Whether Qigong } \\
\text { increases positive affect }\end{array}$ & 1) Affect (SCAS) & 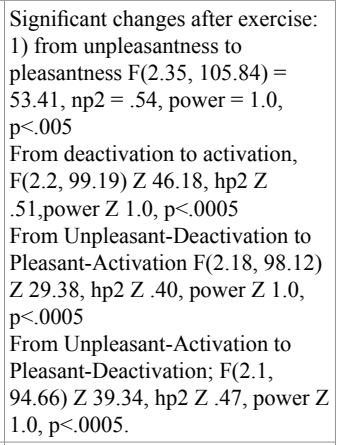 \\
\hline $\begin{array}{l}\text { Overcash } \\
\text { et al. } \\
\text { (2013) } \\
{[52]}\end{array}$ & OS & 38 & US & $\begin{array}{l}57.63(11.3), \\
36-75\end{array}$ & NR & NR & MQ & Pre- and post- design & $\begin{array}{l}\text { Evaluate MQ has effect } \\
\text { on fatigue, depression, } \\
\text { and sleep in cancer } \\
\text { patients and survivors }\end{array}$ & $\begin{array}{l}\text { 1) Fatigue (BFI) } \\
\text { 2) Depression (CES-D) } \\
\text { 3) Sleep (PSQI) }\end{array}$ & $\begin{array}{l}\text { 1) No significant changes in fatigue } \\
(\mathrm{t}=2, \mathrm{p}=0.06) \\
\text { 2) Significantly lower depressive } \\
\text { symptoms ( } \mathrm{t}=3.38, \mathrm{p}<0.05) \\
\text { 3) No significant changes in sleep } \\
\text { quality }(\mathrm{t}=0.85, \mathrm{p}=0.41)\end{array}$ \\
\hline $\begin{array}{l}\text { Kuan } e t \\
\text { al. (2012) } \\
{[51]}\end{array}$ & OS & 66 & US & $75.5(12.4)$ & $\begin{array}{l}\text { QG: } \\
20 \mathrm{M}, \\
12 \mathrm{~F} \\
\mathrm{C:} \\
15 \mathrm{M}, \\
19 \mathrm{~F}\end{array}$ & NR & Ho-gong & $\begin{array}{l}12 \text { weeks, } 5 \text { days/ } \\
\text { week, } 35 \text { min/day } \\
\text { Quasi-experimental, } \\
\text { pre-post test, } \\
\text { nonequivalent control } \\
\text { group design } \\
\text { QG }(\mathrm{n}=32) \text {, control } \\
(\mathrm{n}=34)\end{array}$ & $\begin{array}{l}\text { Test effects of Qigong } \\
\text { exercise program on } \\
\text { physiological and } \\
\text { psychological health of } \\
\text { wheelchair-bound older } \\
\text { adults }\end{array}$ & $\begin{array}{l}\text { 1) Blood pressure } \\
\text { 2) Distal skin temperature } \\
\text { 3) Psychological symptoms } \\
\text { (BSRS-5) }\end{array}$ & $\begin{array}{l}\text { QG versus Control at week } 12 \\
\text { 1) lower BP; systolic: } 103.6 \text { vs. } \\
\text { 128.8, p<.001; diastolic: } 60.0 \text { vs. } \\
73.0, \mathrm{p}<.001) \\
\text { 2) higher distal skin temperature: } \\
\text { 36.1 vs. } 33.8, \mathrm{p}<.001 \\
\text { 3) fewer psychological symptoms: } \\
\text { 1.1 vs. } 6.9, \mathrm{p}<.001\end{array}$ \\
\hline $\begin{array}{l}\text { Johansson } \\
\text { and } \\
\text { Hassmén } \\
(2008) \\
{[54]}\end{array}$ & OS & 41 & Sweden & $56.7(12.4)$ & $\begin{array}{l}6 \mathrm{M}, \\
35 \mathrm{~F}\end{array}$ & $\begin{array}{l}\text { Practicing } \\
\text { for average } \\
6.9(3.8) \\
\text { years } \\
22 \\
\text { participants } \\
\text { were } \\
\text { instructors }\end{array}$ & $\begin{array}{l}\text { Jichu Gong, } \\
\text { Dong Gong } \\
\text { (Biyun } \\
\text { Qigong) }\end{array}$ & $\begin{array}{l}\text { Randomized, cross- } \\
\text { over design between } \\
\text { practicing Qigong } \\
\text { for } 30 \text { minutes or } 60 \\
\text { minutes }\end{array}$ & $\begin{array}{l}\text { Evaluate if length of } \\
\text { Qigong exercise impacts } \\
\text { mood and anxiety }\end{array}$ & $\begin{array}{l}\text { 1) Mood (POMS) } \\
\text { 2) Anxiety (STAI) }\end{array}$ & $\begin{array}{l}30 \text { minutes is sufficient to } \\
\text { produce psychological benefits, } \\
\text { and significant time effects were } \\
\text { found for: } \\
\text { 1) tension } \mathrm{F}(1,40)=50.57, \eta \mathrm{p} 2 \\
=0.56 \text {, power }=1.0, \mathrm{p}<0.0005 ; \\
\text { depression } \mathrm{F}(1,40)=11.84, \eta \mathrm{p} 2 \\
=0.23, \text { power }=0.92, \mathrm{p}<0.001 ; \\
\text { anger } \mathrm{F}(1,40)=8.54, \eta \mathrm{p} 2=0.18, \\
\text { power }=0.81, \mathrm{p}<0.006 \text {; vigor } \mathrm{F}(1 \text {, } \\
40)=29.74, \eta \mathrm{p} 2=0.43, \text { power }= \\
1.0, \mathrm{p}<0.0005 ; \text { fatigue } \mathrm{F}(1,40) \\
=9.91, \eta \mathrm{p} 2=0.20, \text { power }=0.87, \\
\mathrm{p}<0.003 \text {; confusion } \mathrm{F}(1,40)= \\
31.57, \eta \mathrm{p} 2=0.44, \text { power }=1.0, \mathrm{p} \\
<0.0005 \\
\text { 2) anxiety } \mathrm{F}(1,40)=78.01, \eta \mathrm{p} 2= \\
0.66, \text { power }=1.0, \mathrm{p}<0.0005 ;\end{array}$ \\
\hline
\end{tabular}

$\mathrm{SD}=$ Standard deviation; QG=Qigong; OS=Observational study; US=United States; C=Control; NR=Not reported; BSRS-5=Brief Symptom Rating Scale-5 Items; BP=Blood pressure; EQT=External qi therapy; VAS=Visual Analogue Scale; ADL=Activities of Daily Living; FACT-G=Functional Assessment of Cancer Therapy-General; SF-MPQ=Short Form-McGill Pain Questionnaire; MFI-20=Multidimensional Fatigue inventory-20 items; PSQI=Pittsburg Sleep Quality Index; FIQ=Fibromyalgia Impact Questionnaire; BDI-II=Beck Depression InventoryII; OT=observational trial; $\mathrm{CFQ}=$ Chaoyi fanhuan Qigong; PI-NRS=pain intensity numeral rating scale; SF-36=Short Form-36; STAI=State-Trait Anxiety Inventory; LSE=Lorig's selfefficacy scale; MPQ=McGill Pain Questionnaire; TC=Tai Chi; PSQ=Perceived Stress Questionnaire; CES-D=Center for Epidemiologic Studies Depression Scale; MQ=Medical Qigong; BFI=Brief Fatigue Inventory; POMS=Profile of Mood States; SCAS=Swedish Core Affect Scale; BDJ=Baduanjin; SDS=Self-Rated Depression Scale; HAMD=Hamilton Depression Scale

With regard to primary health outcomes, studies indicated mixed to positive results. Cohen et al. [45] found that EQT did not change tumor size among nine women with breast cancer in the U.S. and in China. In an open trial study, preliminary data by Liu et al. [49] showed significant improvement in the average level of blood glucose after a 12-week Qigong exercise program $(-0.32 \pm 0.26 \%$, $\mathrm{p}<.01)$. With regard to musculoskeletal health outcomes, four studies with widely varying methods reported less pain among participants in a Qigong program $[43,44,48,50]$. Sakata et al. [46] found in two separate studies that Qigong significantly increased physical function among relatively health elderly Japanese women through both physiological and physical measurements and also found no significant difference between a Qigong/aerobic exercise regimen versus a Qigong-only exercise regimen. Overall, authors found that Qigong significantly improved depressive symptoms [51-53] and mood or affect [47,54]. Many authors examined psychological symptoms as secondary outcomes and found significant positive results in quality of life [45], depression [44,49,50], anxiety [43,44], and stress [49].

\section{Health effects of Qigong in RCT studies by primary outcome of interest}

Table 4 presents 48 RCT studies which focus on the effects of 
Table 4. RCT of QG by Primary Outcome of Interest.

\begin{tabular}{|c|c|c|c|c|c|c|c|c|c|c|c|c|}
\hline \multirow[t]{2}{*}{ Author (Year) } & \multicolumn{6}{|c|}{ Population Characteristics } & \multirow[t]{2}{*}{ Methods } & \multirow[t]{2}{*}{ Intervention } & \multirow[t]{2}{*}{ Control } & \multirow{2}{*}{$\begin{array}{l}\text { Outcomes of } \\
\text { Interest }\end{array}$} & \multirow{2}{*}{$\begin{array}{l}\text { Instrument, if } \\
\text { applicable }\end{array}$} & \multirow[t]{2}{*}{ Critical Findings } \\
\hline & $\#$ & Country & $\begin{array}{l}\text { Age, Mean } \\
\text { (SD), Range }\end{array}$ & Sex & $\begin{array}{l}\text { Previous QG } \\
\text { Practice }\end{array}$ & $\begin{array}{l}\text { Type of } \\
\text { QG }\end{array}$ & & & & & & \\
\hline \multicolumn{13}{|l|}{ Balance } \\
\hline $\begin{array}{l}\text { Liu et al. } \\
\text { (2015) [64] \# }\end{array}$ & 95 & China & $\begin{array}{l}\text { QG: } 67.1 \\
(6.18) \\
\text { C: } 66.63 \\
(5.98)\end{array}$ & $\begin{array}{l}\text { QG: } 9 \mathrm{M}, 38 \mathrm{~F} \\
\text { C: } 11 \mathrm{M}, 37 \mathrm{~F}\end{array}$ & None & BDJ & \begin{tabular}{|l|}
12 weeks, \\
instruction/ \\
practice \\
with QG \\
professional \\
for two weeks \\
(twice a week, \\
$30-40$ min \\
each), then \\
practice for 10 \\
weeks (twice \\
a week, $30-40$ \\
min each) \\
Data collected \\
at baseline and \\
12 weeks \\
\end{tabular} & $\begin{array}{l}\text { QG and } \\
\text { health } \\
\text { education, } \\
\mathrm{N}=47\end{array}$ & $\begin{array}{l}\text { Walking } \\
\text { and health } \\
\text { education, } \\
\mathrm{N}=48\end{array}$ & $\begin{array}{l}\text { Study efficacy } \\
\text { of BDJ on falls } \\
\text { and balance of } \\
\text { Chinese senior } \\
\text { with chronic } \\
\text { disease }\end{array}$ & $\begin{array}{l}\text { 1) Falls } \\
\text { (MFES) }\end{array}$ & $\begin{array}{l}\text { At } 12 \text { weeks, QG vs. C: } \\
\text { 1) significant } \\
\text { improvement of MFES } \\
\text { scores among intervention } \\
\text { group, } 132.41 \pm 12.59 \text { vs. } \\
123.4 \pm 14.3, \mathrm{p}<.05\end{array}$ \\
\hline $\begin{array}{l}\text { Yang et al. } \\
\text { (2007) [63] }\end{array}$ & 49 & U.S. & $\begin{array}{l}\text { QG: } 80.2 \\
(9.02), 60-97 \\
\text { C: } 80.9 \\
(7.97), 67-94\end{array}$ & $\begin{array}{l}\text { QG: } 5 \mathrm{M}, 28 \mathrm{~F} \\
\text { C: } 5 \mathrm{M}, 11 \mathrm{~F}\end{array}$ & NR & $\begin{array}{l}\text { Tai Chi } \\
\text { Chen style } \\
\text { essential } \\
48 \text { form } \\
\text { and QG } \\
\text { meditation }\end{array}$ & \begin{tabular}{|l|}
6 months, 3 \\
days a week, \\
60 min each \\
session \\
Data taken \\
at baseline, 2 \\
months, and 6 \\
months
\end{tabular} & $\mathrm{N}=33$ & $\begin{array}{l}\text { Wait-list, } \\
\mathrm{N}=16\end{array}$ & $\begin{array}{l}\text { Evaluate } \\
\text { changes } \\
\text { in balance } \\
\text { mechanism } \\
\text { from Tai } \\
\text { Chi Qigong } \\
\text { program }\end{array}$ & $\begin{array}{l}\text { 1) Posture } \\
\text { (SOT) } \\
\text { 2) Stability } \\
\text { (BOS, feet } \\
\text { opening angle) }\end{array}$ & $\begin{array}{l}\text { At } 6 \text { months, between QG } \\
\text { and C: } \\
\text { 1) Relative SOT } \\
\text { vestibular ratios for the } \\
\text { QG group were } 47 \% \\
\text { greater than } \mathrm{C}, \mathrm{p}<0.01 \\
\text { 2) BoS measurements } \\
\text { were } 27 \% \text { greater for the } \\
\text { QG group than } \mathrm{C}, \mathrm{p}<0.01 \text {. } \\
\text { No differences were } \\
\text { observed in feet opening } \\
\text { angle. } \\
\text { For QG group: } \\
\text { 1) Normalized SOT } \\
\text { vestibular ratio scores } \\
\text { increased significantly } \\
\text { by } 34 \% \text { above baseline at } \\
2 \text { months (p<0.01), and } \\
\text { increased } 6 \% \text { between } \\
2 \text { months and } 6 \text { months } \\
(\mathrm{p}>.05) \\
2 \text { ) The normalized BoS } \\
\text { score was } 28 \% \text { above } \\
\text { baseline at T2 (P }<0.01 \text { ) } \\
\text { and was maintained at } \\
30 \% \text { above baseline T6 } \\
(\mathrm{P}<0.01 \text { ). }\end{array}$ \\
\hline $\begin{array}{l}\text { Wenneberg et } \\
\text { al. (2004) [65] }\end{array}$ & 31 & Sweden & $33-80$ & $17 \mathrm{~F}, 19 \mathrm{M}$ & NR & NR & $\begin{array}{l}12 \text { weeks } \\
\text { (Weekend } \\
\text { immersion, } \\
\text { then } 45-50 \text { min } \\
\text { once a week } \\
\text { for } 4 \text { weeks, } \\
\text { then every } \\
\text { other week for } \\
8 \text { weeks with } \\
\text { instructor) } \\
\text { Data collected } \\
\text { at baseline and } \\
12 \text { weeks }\end{array}$ & $\mathrm{N}=16$ & $\begin{array}{l}\text { Wait-List, } \\
\mathrm{N}=15\end{array}$ & $\begin{array}{l}\text { Effects of QG } \\
\text { in patients } \\
\text { with muscular } \\
\text { dystrophy }\end{array}$ & $\begin{array}{l}\text { 1) Balance } \\
\text { (BBS) } \\
\text { 2) QoL (SF- } \\
\text { 36) } \\
\text { 3) Coping } \\
\text { (WCQ) } \\
\text { 4) Depression } \\
\text { (MADRS) }\end{array}$ & $\begin{array}{l}\text { After } 12 \text { weeks, } \\
\text { 1) no between group } \\
\text { difference ( } \mathrm{p}=.128) \\
\text { 2) significant difference } \\
\text { between groups in general } \\
\text { health perceptions after } \\
\text { intervention ( } \mathrm{p}=.027 \text { ), } \\
\text { none in other areas } \\
\text { 3)significant between- } \\
\text { group difference in } \\
\text { positive reappraisal } \\
\text { ( }=.052 \text { ), none in other } \\
\text { areas } \\
\text { 4) no significant } \\
\text { differences between group } \\
\text { or within group }\end{array}$ \\
\hline \multicolumn{13}{|c|}{ Cognitive Function } \\
\hline $\begin{array}{l}\text { Oh et al. (2012) } \\
\text { [66] }\end{array}$ & 81 & Australia & $\begin{array}{l}\text { QG: 64.6 } \\
(12.3) \\
\text { C: } 61.1(11.0)\end{array}$ & $\begin{array}{l}\text { QG: 18F, } \\
18 \mathrm{M} \\
\mathrm{C}: 20 \mathrm{~F}, 20 \mathrm{M}\end{array}$ & None & $\begin{array}{l}\text { MQ } \\
\text { (Daoyin) }\end{array}$ & $\begin{array}{l}10 \text { weeks, two } \\
\text { supervised } 90- \\
\text { min sessions } \\
\text { per week. } \\
\text { Data collected } \\
\text { at baseline and } \\
10 \text { weeks }\end{array}$ & $\mathrm{N}=37$ & $\begin{array}{l}\text { Usual } \\
\text { care, } \\
\mathrm{N}=44\end{array}$ & $\begin{array}{l}\text { Evaluate } \\
\text { effects of MQ } \\
\text { on cognitive } \\
\text { function, } \\
\text { quality of } \\
\text { life, and } \\
\text { inflammation }\end{array}$ & $\begin{array}{l}\text { 1) Cognitive } \\
\text { function } \\
\text { (EORTC-CF } \\
\text { and FACT- } \\
\text { Cog) } \\
\text { 2) QoL } \\
\text { (FACT-G) } \\
\text { 3) } \\
\text { Inflammation } \\
\text { (CRP) }\end{array}$ & $\begin{array}{l}\text { At } 10 \text { weeks, QG } \\
\text { compared to C: } \\
\text { 1) improved cognitive } \\
\text { function (EORTC), mean } \\
\text { difference= }=7.78 \text { (CI, }-0.35 \\
\text { to } 15.92), p=.01 \\
\text { Improved cognitive } \\
\text { function (FACT-Cog), } \\
\text { mean difference= } 4.70 \text { (CI, } \\
-.30 \text { to } 9.71), p=.03 \\
\text { 2) improved total QoL, } \\
\text { mean difference } 12.66 \\
\text { (CI, } 8.00 \text { to } 17.32 \text { ), } \\
\text { p }<.001 \\
\text { 3) less inflammation, } \\
\text { mean difference }=-0.72 \\
\text { (CI, }-1.37 \text { to }-0.07), p=.04 \\
\end{array}$ \\
\hline
\end{tabular}




\begin{tabular}{|c|c|c|c|c|c|c|c|c|c|c|c|c|}
\hline \multicolumn{13}{|l|}{ Diabetes } \\
\hline $\begin{array}{l}\text { Wei and Wu } \\
\text { (2014) [67] \# }\end{array}$ & 60 & China & $\begin{array}{l}54-73 \\
\text { QG: } 63.9 \\
\text { (7.6) } \\
\text { Walking: } 64.8 \\
\text { (5.8) } \\
\text { C: } 65.3(6.0)\end{array}$ & $\begin{array}{l}38 \mathrm{M} \\
22 \mathrm{~F}\end{array}$ & None & BDJ & $\begin{array}{l}12 \text { weeks, at } \\
\text { least } 5 \text { days/ } \\
\text { week, } 3 \text { times } \\
\text { a day } \\
\text { Data collected } \\
\text { at baseline and } \\
12 \text { weeks }\end{array}$ & $\begin{array}{l}\text { QG, N=20 } \\
\text { Walking } \\
\text { group, } \mathrm{N}=20\end{array}$ & $\mathrm{~N}=20$ & $\begin{array}{l}\text { Observe } \\
\text { the clinical } \\
\text { efficacy of } \\
\text { BDJ on type } \\
2 \text { diabetes } \\
\text { patient's health } \\
\text { states. }\end{array}$ & $\begin{array}{l}\text { 1) Diabetes } \\
\text { related health } \\
\text { status (CSSD- } \\
\text { 70) } \\
\text { 2) QoL (SF- } \\
\text { 36) }\end{array}$ & $\begin{array}{l}\text { At } 12 \text { weeks, } Q G \text { versus } \\
\text { control group: } \\
\text { 1) significantly better } \\
\text { diabetes-related health: } \\
85.2 \pm 3.1 \text { vs. } 77.4 \pm 6.2 \text {, } \\
\text { p }<.05 \\
\text { 2) significantly better } \\
\text { quality of life } \\
\text { Physical component: } \\
88 \pm 10.9 \text { vs, } 82.4 \pm 7.4 \text {, } \\
\text { p }<.05 \\
\text { Mental component: } \\
77.7 \pm 9.3 \text { vs. } 67 \pm 7.9, \\
\text { p }<.05\end{array}$ \\
\hline \multicolumn{13}{|l|}{ Pain } \\
\hline $\begin{array}{l}\text { Cai et al. } \\
\text { (2015) [57]\# }\end{array}$ & 60 & China & $\begin{array}{l}\text { QG: } 50.8 \\
(8.0) \\
\text { C: } 49.9(8.0)\end{array}$ & $\begin{array}{l}\text { QG: 16M, } \\
14 \mathrm{~F} \\
\text { C: } 17 \mathrm{M}, 13 \mathrm{~F}\end{array}$ & None & BDJ & $\begin{array}{l}6 \text { months, } \\
\text { twice a day, } 30 \\
\text { min each } \\
\text { VAS data } \\
\text { collected } \\
\text { baseline and } 1 \\
\text { month } \\
\text { Subjective pain } \\
\text { data collected } \\
\text { at } 1 \text { month and } \\
6 \text { months }\end{array}$ & $\mathrm{N}=30$ & $\mathrm{~N}=30$ & $\begin{array}{l}\text { Explore the } \\
\text { effect of BDJ } \\
\text { exercise on the } \\
\text { treatment and } \\
\text { recovery of } \\
\text { patients with } \\
\text { spinal disease. }\end{array}$ & $\begin{array}{l}\text { 1) Pain (VAS) } \\
\text { 2) subjective } \\
\text { pain } \\
\text { assessment }\end{array}$ & 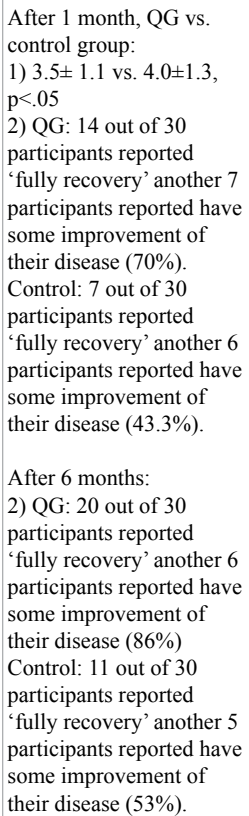 \\
\hline $\begin{array}{l}\text { Wang et al. } \\
\text { (2014) [59] }\end{array}$ & 72 & China & $\begin{array}{l}\text { QG: } 57.06 \\
(8.96) \\
\text { C: } 59.37 \\
(6.51)\end{array}$ & $\begin{array}{l}\text { QG: } 27 \mathrm{~F}, 7 \mathrm{M} \\
\text { C: } 24 \mathrm{~F}, 11 \mathrm{M}\end{array}$ & $\begin{array}{l}\text { None within } \\
6 \text { months }\end{array}$ & BDJ & $\begin{array}{l}6 \text { months }(2 \\
\text { hours a day } \\
\text { training for } \\
\text { first two weeks, } \\
\text { then } 30 \text { min } \\
\text { collective } \\
\text { exercise daily) } \\
\text { Data collected } \\
\text { at baseline, } 3 \\
\text { months, and } 6 \\
\text { months }\end{array}$ & $\mathrm{N}=36$ & $\begin{array}{l}\text { Weekly } \\
30 \text { min } \\
\text { telephone } \\
\text { interview, } \\
\mathrm{N}=36\end{array}$ & $\begin{array}{l}\text { Observe long- } \\
\text { term effects of } \\
\text { regular BDJ } \\
\text { exercises on } \\
\text { chronic neck } \\
\text { pain }\end{array}$ & $\begin{array}{l}\text { 1) Pain (VAS, } \\
\text { NPQ) } \\
\text { 2) SF-36 }\end{array}$ & $\begin{array}{l}\text { At } 6 \text { months, differences } \\
\text { between BDJ and control } \\
\text { 1) less pain (VAS): } 48.97 \\
\text { (18.54) vs. } 57.71 \text { (12.91), } \\
\text { p=.026 } \\
\text { less pain (NPQ): } 20.17 \\
\text { (17.43) vs. } 27.25(9.59) \text {, } \\
\text { p=.04 } \\
\text { 2) all NS except improved } \\
\text { health transition, } 28.03 \text { vs. } \\
\text { 41.77, } p=.002\end{array}$ \\
\hline $\begin{array}{l}\text { Lynch et al. } \\
\text { (2012) [81] }\end{array}$ & 100 & Canada & $\begin{array}{l}\text { QG: } 52.81 \\
(8.91) \\
\text { C: } 52.13 \\
(8.56)\end{array}$ & $\begin{array}{l}\text { QG: } 3 \mathrm{M}, 50 \mathrm{~F} \\
\text { C: } 1 \mathrm{M}, 46 \mathrm{~F}\end{array}$ & None & CFQ & $\begin{array}{l}\text { Training over } \\
3 \text { half-days } \\
\text { followed by } \\
\text { weekly review/ } \\
\text { practice for } 8 \\
\text { weeks } \\
\text { Asked to } \\
\text { practice } 45-60 \\
\text { minutes per } \\
\text { day } \\
\text { Data collected } \\
\text { at baseline, } 6 \\
\text { months }\end{array}$ & $\mathrm{N}=53$ & $\begin{array}{l}\text { Wait-list, } \\
\mathrm{N}=47\end{array}$ & $\begin{array}{l}\text { Effects of CFQ } \\
\text { on pain impact } \\
\text { of fibromyalgia }\end{array}$ & $\begin{array}{l}\text { 1) Pain (PI- } \\
\text { NRS) } \\
\text { 2) } \\
\text { Fibromyalgia } \\
\text { impact (FIQ) } \\
\text { 3) Sleep } \\
\text { (PSQI) }\end{array}$ & $\begin{array}{l}\text { QG group, change from } \\
\text { baseline at } 6 \text { months } \\
\text { 1) }-1.30(2.09), \\
p=.003,38.4 \% \text { saw } \\
\text { clinically meaningful } \\
\text { improvements, } \mathrm{p}=.02 \\
2)-15.19(19.86) \\
p=.003,56.2 \% \text { saw } \\
\text { clinically meaningful } \\
\text { improvements, } \mathrm{p}=.02 \\
\text { 3) }-2.86(3.47), \\
p=.008,49.3 \% \text { saw } \\
\text { clinically meaningful } \\
\text { improvements, }=.01\end{array}$ \\
\hline
\end{tabular}




\begin{tabular}{|c|c|c|c|c|c|c|c|c|c|c|c|c|}
\hline $\begin{array}{l}\text { Wei et al. } \\
\text { (2012) [115] \# }\end{array}$ & 62 & China & $\begin{array}{l}\text { QG: } 29.39 \\
(11.35), 15-58 \\
\text { C: } 31.97 \\
(12.15), 18-61\end{array}$ & $\begin{array}{l}\text { QG: } 25 \mathrm{M}, 6 \mathrm{~F} \\
\text { C: } 26 \mathrm{M}, 5 \mathrm{~F}\end{array}$ & none & BDJ & $\begin{array}{l}3 \text { months, } \\
\text { twice a day, } \\
15-20 \text { minutes } \\
\text { each session } \\
\text { Data collected } \\
\text { at baseline and } \\
3 \text { months }\end{array}$ & N31 & $\mathrm{N}=31$ & $\begin{array}{l}\text { Explore the } \\
\text { effect of BDJ } \\
\text { exercise on the } \\
\text { inflammation } \\
\text { index of AS } \\
\text { patients }\end{array}$ & $\begin{array}{l}\text { 1) Pain (VAS) } \\
\text { 2) Disease } \\
\text { symptoms } \\
\text { (ASAS 20) } \\
\\
\end{array}$ & $\begin{array}{l}\text { At } 3 \text { months, differences } \\
\text { between QG and control } \\
\text { 1) Pain: } 1.68 \pm 0.60 \text { vs } \\
2.48 \pm 0.69, \mathrm{p}<.05 \\
\text { 2) Physical } \\
\text { Function: } 2.13 \pm 0.7 \text { vs } \\
3.25 \pm 0.91, \mathrm{p}<.05 \\
\text { Thoracic } \\
\text { activity } \square 4.3 \pm 1.32 \text { vs } \\
3.66 \pm 1.07, \mathrm{p}<.05 \\
\text { Schober back flex: } \\
4.69 \pm 1.39 \text { vs } 3.79 \pm 1.26 \\
\mathrm{p}<.05\end{array}$ \\
\hline $\begin{array}{l}\text { von Trott et al. } \\
\text { (2009) [69] }\end{array}$ & 117 & Germany & $76(8), 55+$ & $6 \mathrm{M}, 111 \mathrm{~F}$ & NR & NR & $\begin{array}{l}3 \text { months, } 24 \\
\text { sessions total, } \\
45 \text { minutes } \\
\text { each of Qigong } \\
\text { or exercise } \\
\text { therapy } \\
\text { Data collected } \\
\text { and baseline, } 3 \\
\text { months, and } 6 \\
\text { months }\end{array}$ & $\begin{array}{l}\text { Qigong } \\
\text { therapy, } \\
\mathrm{N}=38 \\
\text { Exercise } \\
\text { therapy, } \\
\mathrm{N}=39\end{array}$ & $\begin{array}{l}\text { Waiting } \\
\text { list } \\
\text { control, } \\
\mathrm{N}=32\end{array}$ & $\begin{array}{l}\text { Evaluate } \\
\text { effectiveness of } \\
\text { QG compared } \\
\text { to exercise } \\
\text { therapy and no } \\
\text { treatment }\end{array}$ & $\begin{array}{l}\text { 1) Average } \\
\text { neck pain } \\
\text { (VAS) } \\
\text { 2) Neck pain } \\
\text { and disability } \\
\text { (NPAD) } \\
\text { 3) Depression } \\
\text { (ADS) }\end{array}$ & $\begin{array}{l}\text { at } 3 \text { months, NS } \\
\text { difference between QG } \\
\text { and } \mathrm{C}(95 \% \mathrm{CI}) \\
\text { 1) }-11.0(-24.0 \text { to } 2.1) \text {, } \\
\mathrm{p}=.10 \\
\text { 2) }-6.7(-15.4 \text { to } 2.1), \\
\mathrm{p}=.14 \\
\text { 3) }-1.0(-5.2 \text { to } 3.1), \mathrm{p}=.62\end{array}$ \\
\hline $\begin{array}{l}\text { Lansinger et al. } \\
\text { (2007) [68] }\end{array}$ & 122 & Sweden & $\begin{array}{l}\text { QG: } 44.9 \\
\text { (12.3), 20-62 } \\
\text { C: } 42.8(1.4) \text {, } \\
21-65\end{array}$ & $\begin{array}{l}\text { Q: } 44 \mathrm{~F}, 16 \mathrm{M} \\
\text { C: } 42 \mathrm{~F}, 20 \mathrm{M}\end{array}$ & NR & Biyun & \begin{tabular}{l|}
3 months, $1-2$ \\
sessions a \\
week, 1 hour \\
(10-12 session \\
total) \\
Data collected \\
at baseline, \\
3 months, 6 \\
months after \\
intervention, \\
and 12 \\
months after \\
intervention
\end{tabular} & $\mathrm{N}=60$ & $\begin{array}{l}\text { Exercise } \\
\text { Therapy, } \\
\mathrm{n}=62\end{array}$ & $\begin{array}{l}\text { Effect on long- } \\
\text { term neck pain }\end{array}$ & $\begin{array}{l}\text { 1) Pain (diary, } \\
\text { VAS, NDI) } \\
\\
\end{array}$ & $\begin{array}{l}\text { 1) No differences between } \\
\text { groups for NP frequency, } \\
\text { average NP in the most } \\
\text { recent week, current NP, } \\
\text { NP diary, and NDI. } \\
\text { Patients improved } \\
\text { immediately after } \\
\text { intervention and at the 6- } \\
\text { and } 12 \text {-month follow-ups: } \\
\text { above } 50 \% \text { for average } \\
\text { NP in the most recent } \\
\text { week, NP diary, NDI. and } \\
\text { current NP (not for the } \\
\text { time immediately after the } \\
\text { intervention period). }\end{array}$ \\
\hline $\begin{array}{l}\text { Yang et al. } \\
\text { (2005) [116] }\end{array}$ & 40 & Korea & $\begin{array}{l}\text { QG: } 72.58 \\
(5.41) \\
\text { C: } 72.67 \\
(7.49)\end{array}$ & $\begin{array}{l}\text { QG: 13F, 6M } \\
\text { C:19F, 2M }\end{array}$ & NR & EQT & $\begin{array}{l}4 \text { weeks, twice } \\
\text { a week } \\
\text { QG: } 20 \\
\text { minutes } \\
\text { receiving Qi } \\
\text { C: lay in } \\
\text { similar position } \\
\text { but without Qi } \\
\text { Data collected } \\
\text { at baseline, } \\
\text { week 1, week } \\
\text { 2, week 3, } \\
\text { week 4, and } \\
\text { week 6 }\end{array}$ & $\mathrm{N}=19$ & $\begin{array}{l}\text { Wait-list, } \\
\mathrm{N}=21\end{array}$ & $\begin{array}{l}\text { Effects of EQT } \\
\text { on pain and } \\
\text { mood states }\end{array}$ & $\begin{array}{l}\text { 1) Pain (VAS) } \\
\text { 2) Mood } \\
\text { (POMS) } \\
\end{array}$ & $\begin{array}{l}\text { 1) pain intensity } \\
\text { decreased linearly over } \\
\text { time in } \mathrm{QG} \text { group but } \\
\text { not in control group. } \\
\text { Significant between- } \\
\text { group time improvement } \\
\text { in QG versus control } \\
{[\mathrm{F}(5,190)=13.8, \mathrm{p}<.0001 \text {, }} \\
\mathrm{HF}-\mathrm{e}=.95] \\
\text { 2) positive mood: } \\
\text { significant between- } \\
\text { group time improvement } \\
\text { in } \mathrm{QG} \text { versus control } \\
{[\mathrm{F}(5,190)=22 / 1, \mathrm{p}<.0001] \text {; }} \\
\text { gradually increased in } \mathrm{QG} \\
\text { but decreased in control } \\
\text { Negative mood: } \\
\text { significant between- } \\
\text { group time improvement } \\
\text { in } \mathrm{QG} \text { versus control } \\
{[\mathrm{F}(5,190)=10.9, \mathrm{p}<.0001,} \\
\mathrm{HF}-\mathrm{e}=.69] .\end{array}$ \\
\hline $\begin{array}{l}\text { Lee } \text { et al. } \\
\text { (2001) [117] }\end{array}$ & 40 & Korea & $\begin{array}{l}\text { QG: } 73.05 \\
(5.67) \\
\text { C: } 72.20 \\
(7.36)\end{array}$ & $\begin{array}{l}\text { QG: } 14 \mathrm{~F}, 6 \mathrm{M} \\
\text { C: } 18 \mathrm{~F}, 2 \mathrm{M}\end{array}$ & NR & CSDB & $\begin{array}{l}2 \text { weeks, qi } \\
\text { therapy twice } \\
\text { a week for } 10 \\
\text { minutes (total } 4 \\
\text { times) } \\
\text { Performed by } \\
\text { certified Qi } \\
\text { therapist } \\
\text { Data collected } \\
\text { at pre-therapy, } \\
\text { one week, and } \\
\text { two weeks }\end{array}$ & $\mathrm{N}=20$ & $\begin{array}{l}\mathrm{N}=20 \\
\text { Received } \\
\text { general } \\
\text { care in } \\
\text { the same } \\
\text { frequency }\end{array}$ & $\begin{array}{l}\text { Assess effects } \\
\text { of Qi therapy } \\
\text { on reducing } \\
\text { pain and } \\
\text { enhancing } \\
\text { mood states }\end{array}$ & $\begin{array}{l}\text { 1) Pain level } \\
\text { (VAS) } \\
\text { 2) Mood states } \\
\text { (POMS) } \\
\\
\\
\\
\end{array}$ & $\begin{array}{l}\text { Significant group } x \text { time } \\
\text { interaction and better } \\
\text { scores in } \mathrm{QG} \text { group vs. } \\
\text { control } \\
\text { 1) Positive mood: } \mathrm{F}(2, \\
76)=21.29, \mathrm{p}=.0001 ; \mathrm{QG} \\
\text { vs. Control: } 9.25 \pm 4.13 \text { vs. } \\
3.35 \pm 2.85, \mathrm{p}<.005 \\
\text { Negative mood: } \\
\mathrm{F}(2,76)=2.93, \mathrm{p}=.06 ; \mathrm{QG} \\
\text { vs. } \text { Control: } 16.90 \pm 11.15 \\
\text { vs. } 25.20 \pm 16.61, \mathrm{NS} \\
\text { 2) Pain: } \mathrm{F}(2,76)=9.379, \\
\mathrm{p}<.0001) \\
\mathrm{QG} \text { group has lower pain } \\
\text { than control at } 2 \text { weeks } \\
(p<.005)\end{array}$ \\
\hline
\end{tabular}




\begin{tabular}{|c|c|c|c|c|c|c|c|c|c|c|c|c|}
\hline $\begin{array}{l}\text { Wang et al. } \\
\text { (2015) [118]\# }\end{array}$ & 26 & China & $\begin{array}{l}\text { 60+ } \\
\text { QG: } 66.79 \\
(4.76) \\
\text { C: } 65.59 \\
(3.59)\end{array}$ & $\begin{array}{l}\text { QG: 7M, 6F } \\
\text { C: } 3 \mathrm{M}, 10 \mathrm{~F}\end{array}$ & None & YJJ & $\begin{array}{l}12 \text { weeks, QG } \\
\text { practice } 3 \times \text { a } \\
\text { week, } 1 \text { hour } \\
\text { each } \\
\text { QG participants } \\
\text { received } \\
1 \text { week of } \\
\text { training prior to } \\
\text { intervention } \\
\text { Data collected } \\
\text { at baseline and } \\
12 \text { weeks }\end{array}$ & $\mathrm{N}=13$ & $\mathrm{~N}=13$ & $\begin{array}{l}\text { Explore the } \\
\text { effect of YJJ } \\
\text { exercise on the } \\
\text { prevention of } \\
\text { Skeletal muscle } \\
\text { weakness } \\
\text { among senior } \\
\text { adults }\end{array}$ & $\begin{array}{l}\text { 1) Physical } \\
\text { function (Knee } \\
\text { extensor/ } / \\
\text { flexor peak } \\
\text { torque, peak } \\
\text { torque/body } \\
\text { weight, and } \\
\text { average } \\
\text { power) } \\
\end{array}$ & $\begin{array}{l}\text { At } 12 \text { weeks, QG vs. } \\
\text { control: } \\
\text { 1) Statistically significant } \\
\text { improvement of } \\
\text { extensor peak torque } \\
60^{\circ} / \mathrm{s}(96.35 \pm 31.18 \text { vs. } \\
76.23 \pm 24.39) \text {, extensor } \\
\text { peak torque/body weight } \\
60^{\circ} / \mathrm{s}(141.03 \pm 33.3 \\
\text { vs. } 113.05 \pm 33.3) \text {, } \\
\text { extensor average power } \\
60^{\circ} / \mathrm{s}(49.1 \pm 12.68 \\
\text { vs. } 40.1 \pm 11.69) \text {, and } \\
\text { extensor average power } \\
180^{\circ} / \mathrm{s}(49.63 \pm 16.65 \text { vs. } \\
36.75 \pm 13.8) \text { among the } \\
\text { intervention group, } \mathrm{p}<.05\end{array}$ \\
\hline $\begin{array}{l}\text { Xiao and } \\
\text { Zhuang (2015) } \\
{[62]}\end{array}$ & 100 & China & $\begin{array}{l}\text { QG: } 68.17 \\
(2.27) \\
\text { C: } 66.52 \\
(2.13)\end{array}$ & $\begin{array}{l}\text { QG: } \\
68.75 \% \mathrm{M} \\
\text { C: } 70.83 \% \mathrm{M}\end{array}$ & None & BDJ & $\begin{array}{l}\text { Four } 45 \text {-min } \\
\text { sessions/week, } \\
\text { daily walking } \\
30 \text { min for } 6 \\
\text { months } \\
\text { Data collected } \\
\text { at enrollment, } \\
\text { discharge from } \\
\text { rehab program } \\
\text { and } 6 \text { months }\end{array}$ & $\mathrm{N}=50$ & $\begin{array}{l}\text { Independent } \\
\text { walking, } \\
\mathrm{N}=50\end{array}$ & $\begin{array}{l}\text { Investigate } \\
\text { effectiveness } \\
\text { of BDJ on } \\
\text { symptoms } \\
\text { related to gait, } \\
\text { functional } \\
\text { mobility and } \\
\text { sleep }\end{array}$ & $\begin{array}{l}\text { 1) Fatigue } \\
\text { (UPDRS) } \\
\text { 2) Functional } \\
\text { mobility } \\
\text { (BBS, 6MWT, } \\
\text { TUG) } \\
\text { 3) Sleep } \\
\text { (PDS) } \\
\text { 4) Gait (Vicon } \\
512 \text { motion } \\
\text { capture } \\
\text { system, } \\
\text { Freezing of } \\
\text { Gait) } \\
\end{array}$ & $\begin{array}{l}\text { Across } 6 \text { months, } \\
\text { comparing QG and } \\
\text { control: } \\
\text { 1) significant group } \times \\
\text { time interactions, with the } \\
\text { QG showing a significant } \\
\text { decrease in impairment } \\
\text { measured by the UPDRS- } \\
\text { III score ( } \mathrm{P}=0.038) \text {. } \\
\text { 2) significant group } \times \\
\text { time interactions, with } \\
\text { the QG showing greater } \\
\text { improvements in the BBS } \\
(\mathrm{P}=0.037) \text { and } 6 \mathrm{MW} \\
(\mathrm{P}=0.045) \text {, and greater } \\
\text { decrease in the TUG (P } \\
=0.028) \\
\text { 3) significant group } x \\
\text { time interactions, with } \\
\text { QG showing significant } \\
\text { decrease in PDSS-2 total } \\
\text { score ( }=.045) \\
\text { 4) significant group } \times \\
\text { time interactions, with the } \\
\text { QG showing a significant } \\
\text { increase in the gait speed } \\
(\mathrm{p}=.02)\end{array}$ \\
\hline $\begin{array}{l}\text { Xiao and } \\
\text { Zhuang (2015) } \\
\text { [61] }\end{array}$ & 126 & China & $\begin{array}{l}71.1(2.7), \\
65-85 \\
\text { QG: } 72.2 \\
(1.7) \\
\text { C: } 70.9(1.4)\end{array}$ & $\begin{array}{l}\text { QG: } 82.1 \% \mathrm{M} \\
\text { C: } 93.6 \% \mathrm{M}\end{array}$ & None & LQG & $\begin{array}{l}6 \text { months, four } \\
45 \text { minute } \\
\text { sessions per } \\
\text { week } \\
\text { Data collected } \\
\text { at baseline and } \\
6 \text { months }\end{array}$ & $\mathrm{N}=63$ & $\begin{array}{l}30 \text { min } \\
\text { walk } \\
\text { daily, } \\
\mathrm{N}=63\end{array}$ & $\begin{array}{l}\text { Investigate } \\
\text { effectiveness } \\
\text { of LQG in } \\
\text { promoting } \\
\text { physical and } \\
\text { psychosocial } \\
\text { function in } \\
\text { individuals } \\
\text { with COPD }\end{array}$ & $\begin{array}{l}\text { 1) Functional } \\
\text { capacity } \\
\text { (6MWT) } \\
\text { 2) General } \\
\text { health (SF-36) } \\
\\
\end{array}$ & $\begin{array}{l}\text { 1) Significant group by } \\
\text { time interactions, with } \\
\text { the QG group showing } \\
\text { greater improvements on } \\
\text { the } 6 \mathrm{MW} \text { (p=.04) over the } \\
6 \text {-month study period than } \\
\text { controls, and significant } \\
\text { improvement for } \mathrm{QG} \\
\text { group between baseline } \\
\text { and } 6 \text { months }(301.0 \pm 10.9 \\
\text { vs. } 321.5 \pm 15.5, \mathrm{p}=.02) \\
\text { 2) Non-significant group } \\
\text { by time interactions } \\
\text { between } \mathrm{QG} \text { and } \\
\text { control over } 6 \text { months } \\
\text { (p=.54), and significant } \\
\text { improvement for } \mathrm{QG} \\
\text { group }(43.9 \pm 3.5 \mathrm{vs.} \\
51.8 \pm 5.6, \mathrm{p}<.001)\end{array}$ \\
\hline $\begin{array}{l}\text { Li et al. (2014) } \\
\text { [119] }\end{array}$ & 110 & China & $\begin{array}{l}34.2(14.6) \text {, } \\
20-59 \\
\text { QG: } 35.5 \\
C: 32.9\end{array}$ & $\begin{array}{l}\text { QG: 19M, } \\
39 \mathrm{~F} \\
\text { C: } 17 \mathrm{M}, 38 \mathrm{~F}\end{array}$ & NR & BDJ & $\begin{array}{l}16 \text { weeks, QG } \\
3 \text { times or more } \\
\text { each week, } \\
30-60 \text { minutes } \\
\text { each time } \\
\text { QG training 2 } \\
\text { weeks prior to } \\
\text { intervention }\end{array}$ & $\mathrm{N}=55$ & $\begin{array}{l}\text { Wait-list } \\
\mathrm{N}=55\end{array}$ & $\begin{array}{l}\text { Effects of BDJ } \\
\text { on promoting } \\
\text { physical fitness }\end{array}$ & $\begin{array}{l}\text { 1) Physical } \\
\text { function (SR, } \\
\text { ES, aerobic } \\
\text { endurance) }\end{array}$ & $\begin{array}{l}\text { 1) SR: better physical } \\
\text { fitness posttest-pretest } \\
\text { scores, t=3.46, p=.001 } \\
\text { ES: worse time but NS } \\
\text { Aerobic endurance: } \\
\text { improved but NS }\end{array}$ \\
\hline
\end{tabular}




\begin{tabular}{|c|c|c|c|c|c|c|c|c|c|c|c|c|}
\hline $\begin{array}{l}\text { Amano et al. } \\
\text { (2013) [79] }\end{array}$ & 21 & US & $\begin{array}{l}\text { TC: } 64 \text { (13) } \\
\text { QG: } 68 \text { (7) }\end{array}$ & $\begin{array}{l}\text { TC: } 7 \mathrm{M}, 5 \mathrm{~F} \\
\text { C: } 7 \mathrm{M}, 2 \mathrm{~F}\end{array}$ & None & NR & $\begin{array}{l}16 \text { weeks, } 2 x \\
\text { week, } 60 \text { mins/ } \\
\text { session for both } \\
\text { TC and QG }\end{array}$ & $\begin{array}{l}\text { Tai Chi } \\
\text { (Yang-style), } \\
\mathrm{N}=12\end{array}$ & $\begin{array}{l}\text { QG } \\
\text { meditation, } \\
\mathrm{N}=9\end{array}$ & $\begin{array}{l}\text { Impact of Tai } \\
\text { Chi vs. Qigong } \\
\text { on gait and gait } \\
\text { performance }\end{array}$ & $\begin{array}{l}\text { 1) Gait } \\
\text { initiation } \\
\text { (center-of- } \\
\text { pressure } \\
\text { measures) } \\
\text { 2) Gait } \\
\text { performance } \\
\text { (cadence, } \\
\text { gait velocity, } \\
\text { step length, } \\
\text { step duration, } \\
\text { swing time, } \\
\text { double lib } \\
\text { support } \\
\text { time, gait } \\
\text { asymmetry) } \\
\text { 3) } \\
\text { Parkinsonian } \\
\text { disabilities } \\
\text { (UPDRS-III) }\end{array}$ & $\begin{array}{l}\text { 1) significantly shifted } \\
\text { their COP more toward } \\
\text { the initial swing limb after } \\
\text { the } 16 \text {-week period when } \\
\text { compared to the TC group } \\
(\mathrm{p}<0.01, \eta 2=0.39) \text {. } \\
\text { 2) no significant } \\
\text { difference between groups } \\
\text { in any variables (ps }>.05 \text { ) } \\
\text { 3) no significant } \\
\text { difference between groups } \\
(\mathrm{ps}>.05)\end{array}$ \\
\hline $\begin{array}{l}\text { Chan et al. } \\
\text { (2011) [70] }\end{array}$ & 206 & $\mathrm{HK}$ & $73,55-88$ & $\begin{array}{l}\text { TCQ: 69M, } \\
\text { 1F } \\
\text { Exercise: } \\
61 \mathrm{M}, 8 \mathrm{~F} \\
\text { C: } 58 \mathrm{M}, 9 \mathrm{~F}\end{array}$ & None & $\begin{array}{l}13 \text { form } \\
\text { TCQ }\end{array}$ & $\begin{array}{l}3 \text { months } \\
\text { TCQ: Two 60- } \\
\text { min sessions } \\
\text { per week } \\
\text { Exercise: } \\
\text { pursed lip and } \\
\text { diaphragmatic } \\
\text { breathing } \\
\text { Data collected } \\
\text { at baseline and } \\
3 \text { months }\end{array}$ & $\begin{array}{l}\text { TCQ, N=70 } \\
\text { Exercise, } \\
\mathrm{N}=69 \\
\\
\end{array}$ & $\begin{array}{l}\text { Control, } \\
\mathrm{N}=67\end{array}$ & $\begin{array}{l}\text { Evaluate the } \\
\text { effectiveness } \\
\text { of TCQ in } \\
\text { enhancing } \\
\text { respiratory } \\
\text { functions } \\
\text { and activity } \\
\text { tolerance in } \\
\text { individuals } \\
\text { with COPD }\end{array}$ & $\begin{array}{l}\text { 1) Lung } \\
\text { functions: Pre- } \\
\text { broncholator } \\
\text { spirometry } \\
\text { 2) 6-min walk } \\
\text { test } \\
\text { 3) Dyspnoea } \\
\text { and fatigue: } \\
\text { Borg scale } \\
\text { 4) Oxygen } \\
\text { saturation }\end{array}$ & 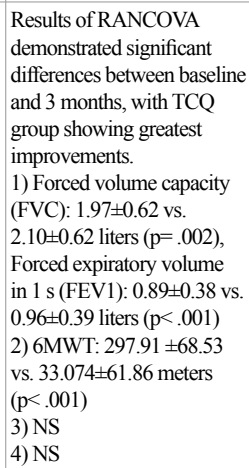 \\
\hline $\begin{array}{l}\text { Maddali } e t a l \text {. } \\
\text { (2011) [120] }\end{array}$ & 30 & Italy & $\begin{array}{l}\text { G1: } 56.56 \\
(9.1) \\
\text { G2: } 57.91 \\
(13.50)\end{array}$ & NR & NR & $\begin{array}{l}\text { Dan tien, } \\
\text { Zhang } \\
\text { zhuang, } \\
\text { Flying } \\
\text { Pheonix }\end{array}$ & $\begin{array}{l}7 \text { weeks, } 2 \\
\text { sessions/ } \\
\text { week for first } \\
\text { three weeks, } 1 \\
\text { session/week } \\
\text { for weeks } \\
4-7 \text { (total } 10 \\
\text { sessions) } \\
\text { Cross-over } \\
\text { design with 1 } \\
\text { week interval } \\
\text { Ressegaier } \\
\text { Method session } \\
\text { is } 60 \text { min } \\
\text { QG is } 45 \text { min } \\
\text { Data collected } \\
\text { at baseline, } \\
\text { week } 7 \text { (end } \\
\text { intervention 1), } \\
\text { week } 15 \text { (end } \\
\text { intervention 2), } \\
\text { week } 27\end{array}$ & $\begin{array}{l}\text { Ressegaier } \\
\text { Method, } \\
\mathrm{N}=15 \\
\mathrm{QG}, \mathrm{N}=15 \\
\\
\end{array}$ & None & $\begin{array}{l}\text { Evaluate } \\
\text { Ressegaier } \\
\text { method } \\
\text { and QG in } \\
\text { fibromyalgia } \\
\text { rehabilitation }\end{array}$ & $\begin{array}{l}\text { 1) disability } \\
\text { (FIQ) } \\
\text { 2) Pain (NRS) } \\
\text { 3) } \\
\text { Psychological } \\
\text { outcomes } \\
\text { (HADS) }\end{array}$ & 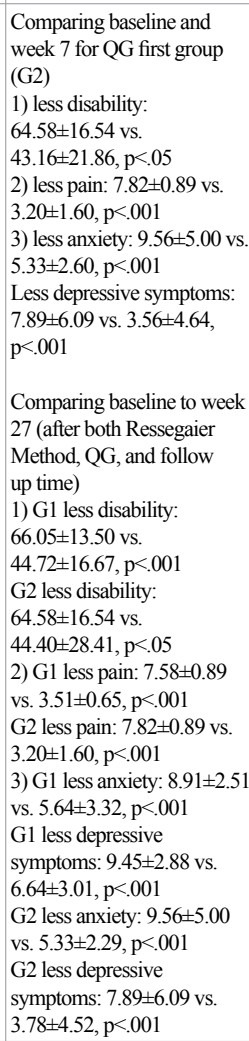 \\
\hline
\end{tabular}




\begin{tabular}{|c|c|c|c|c|c|c|c|c|c|c|c|c|}
\hline $\begin{array}{l}\text { Chen et al. } \\
\text { (2008) [55] }\end{array}$ & 112 & US & $\begin{array}{l}\text { H1: } 63.9(9.7) \\
\text { H2: } 58.8(7.0) \\
\text { C: } 62.9(9.2)\end{array}$ & $\begin{array}{l}\mathrm{H} 1: 14 \mathrm{M}, 31 \mathrm{~F} \\
\mathrm{H} 2: 3 \mathrm{M}, 9 \mathrm{~F} \\
\mathrm{C}: 13 \mathrm{M}, 36 \mathrm{~F}\end{array}$ & $\begin{array}{l}\text { Experience } \\
\text { with some } \\
\text { CAM } \\
\text { therapies, } \\
\text { none with } \\
\text { EQT }\end{array}$ & . & $\begin{array}{l}3 \text { weeks, 5-6 } \\
\text { sessions. Each } \\
\text { healer had } \\
\text { a different } \\
\text { technique } \\
\text { H1: 4-7 } \\
\text { minutes } \\
\text { H2: } 5-10 \\
\text { minutes } \\
\text { C: Chinese } \\
\text { man without } \\
\text { experience } \\
\text { mimicked EQT } \\
\text { movements } \\
\text { Data collected } \\
\text { at baseline, } 3 \\
\text { weeks, and 3 } \\
\text { months }\end{array}$ & $\begin{array}{l}\mathrm{H} 1: \mathrm{N}=45 \\
\mathrm{H} 2 \mathrm{n}=12 \\
\end{array}$ & $\begin{array}{l}\text { Sham } \\
\text { treatment, } \\
\mathrm{N}=52\end{array}$ & $\begin{array}{l}\text { Effects of } \\
\text { EQT on } \\
\text { osteoarthritis } \\
\text { pain and } \\
\text { functional }\end{array}$ & $\begin{array}{l}\text { 1) } \\
\text { Osteoarthritis } \\
\text { pain and } \\
\text { function } \\
\text { (WOMAC) }\end{array}$ & $\begin{array}{l}\text { 1) No difference between } \\
\text { sham and healer } 1 \\
\text { Difference between sham } \\
\text { group and healer } 2 \text { for } \\
\text { pain }(\mathrm{p}<.01) \text {, functionality } \\
(<.01) \text { and total WOMAC } \\
\text { scores ( }<<.01) \text { at } 3 \text { month } \\
\text { follow up } \\
\text { Belief in CAM therapy } \\
\text { was a significant covariate } \\
\text { in predicting treatment } \\
\text { outcome immediately } \\
\text { after treatment }(\mathrm{p}<.05)\end{array}$ \\
\hline $\begin{array}{l}\text { Pippa et al. } \\
\text { (2007) [121] }\end{array}$ & 43 & Italy & $68(8)$ & $30 \mathrm{M}, 13 \mathrm{~F}$ & NR & . & $\begin{array}{l}16 \text { weeks of } \\
\text { intervention } \\
\text { QG: two } \\
90 \text {-minute } \\
\text { training } \\
\text { sessions per } \\
\text { week, } 32 \text { total } \\
\text { Follow-up } 16 \\
\text { weeks after } \\
\text { intervention }\end{array}$ & $\mathrm{N}=22$ & $\begin{array}{l}\text { Wait-list, } \\
\mathrm{n}=21\end{array}$ & $\begin{array}{l}\text { Effects of QG } \\
\text { on functional } \\
\text { capacity }\end{array}$ & $\begin{array}{l}\text { 1) Physical } \\
\text { function } \\
\text { (6MWT) }\end{array}$ & $\begin{array}{l}\text { 1) Significant } \\
\text { improvement in QG } \\
\text { group versus control } \\
\text { group at two time points: } \\
\text { pre-training versus post- } \\
\text { training ( } \mathrm{p}<.001) \text { and } \\
\text { pre-training and follow-up } \\
(\mathrm{p}=.008)\end{array}$ \\
\hline $\begin{array}{l}\text { Burini } \text { et al. } \\
\text { (2006) [122] }\end{array}$ & 26 & Italy & $65.2(6.5)$ & $9 \mathrm{M}, 17 \mathrm{~F}$ & NR & . & $\begin{array}{l}\text { Crossover } \\
\text { design (total } 22 \\
\text { weeks): } \\
7 \text { weeks, } 20 \\
\text { sessions of } 50 \\
\text { min, } 3 \text { days } \\
\text { weekly } \\
8 \text { weeks of no } \\
\text { treatment } \\
7 \text { weeks, } 20 \\
\text { sessions of } \\
50 \text { min, } 3 \\
\text { days weekly } \\
\text { of remaining } \\
\text { treatment } \\
\text { Data collected } \\
\text { at baseline, } \\
\text { after first } \\
\text { intervention, } \\
\text { after no } \\
\text { treatment, } \\
\text { after second } \\
\text { treatment }\end{array}$ & $\begin{array}{l}\text { QG, N=15 } \\
\text { Aerobic } \\
\text { Training } \\
\text { sessions, } \\
\mathrm{N}=11\end{array}$ & None & $\begin{array}{l}\text { Effects of QG } \\
\text { versus aerobic } \\
\text { training in } \\
\text { subjects with } \\
\text { Parkinson's }\end{array}$ & $\begin{array}{l}\text { 1) Impairment } \\
\text { from } \\
\text { Parkinson's } \\
\text { (UPDRS) } \\
\text { 2) Depression } \\
\text { (BDS) } \\
\text { 3) Physical } \\
\text { function } \\
\text { (6MWT) }\end{array}$ & $\begin{array}{l}\text { 1-2) All changes after } \\
\mathrm{QG} \text { training were not } \\
\text { significant, } \mathrm{p}>0.05 \\
\text { 3) Group } \mathrm{x} \text { time } \\
\text { differences significant: } \\
\mathrm{F}=5.4, \mathrm{p}=.002 \\
\text { Aerobic training group } \\
\text { showed significant } \\
\text { improvements }=-2.7, \\
\mathrm{p}=.005 \text {, while } \mathrm{QG} \\
\text { group changes were not } \\
\text { significant }\end{array}$ \\
\hline $\begin{array}{l}\text { Schmitz } \square \\
\text { Hübsch et al. } \\
\text { (2006) [58] }\end{array}$ & 56 & Germany & $\begin{array}{l}\text { QG: 64 (8) } \\
\text { C: } 63(8)\end{array}$ & $\begin{array}{l}\text { QG: } 24 \mathrm{M}, 8 \mathrm{~F} \\
\text { C: } 19 \mathrm{M}, 5 \mathrm{~F}\end{array}$ & None & $\begin{array}{l}\text { "Frolic of } \\
\text { the crane", } \\
\text { sitting } \\
\text { BDJ } \\
\end{array}$ & $\begin{array}{l}24 \text { weeks (Two } \\
\text { courses of } 8 \\
\text { weeks with an } \\
8 \text {-week pause } \\
\text { in between), } 90 \\
\text { minutes each, } \\
16 \text { visits total, } \\
1 \mathrm{X} / \text { week } \\
\text { Encouraged } \\
\text { to practice at } \\
\text { home } \\
\text { Data collected } \\
\text { at baseline, } \\
3 \text { months, } 6 \\
\text { months, and } 12 \\
\text { months }\end{array}$ & $\mathrm{N}=32$ & $\mathrm{~N}=24$ & $\begin{array}{l}\text { Evaluate } \\
\text { effects of } \\
\text { QG on motor } \\
\text { symptoms of } \\
\text { Parkinson's } \\
\text { Disease }\end{array}$ & $\begin{array}{l}\text { 1) Motor } \\
\text { symptoms } \\
\text { (UPDRS-III) } \\
\text { 2) Quality of } \\
\text { life (PDQ-39) } \\
\text { 3) Depression } \\
\text { (MADRS) } \\
\\
\end{array}$ & $\begin{array}{l}\text { 1) The proportion of } \\
\text { patients who improved } \\
\text { in UPDRS- III, was } \\
\text { significantly greater in } \\
\mathrm{QG} \text { group at } 3 \text { months } \\
(\mathrm{P}=0.0080) \text {, while at } 6 \\
\text { months ( } \mathrm{P}=0.0503 \text { ) and } \\
12 \text { months (P= }(\mathrm{P}=0.635 \text { ) was } \\
\text { not significant. } \\
\text { 2) no significant between- } \\
\text { group differences, data } \\
\text { not reported } \\
\text { 3) The prevalence of mild } \\
\text { or moderate depression } \\
\text { was } 48 \% \text { in the } \mathrm{QG} \\
\text { group and } 41 \% \text { in the } \\
\text { control group at baseline } \\
\text { compared with } 33 \% \text { in } \\
\text { both groups at } 6 \text { months } \\
\text { Proportions of patients } \\
\text { using antidepressants } \\
\text { were } 19 \% \text { (QG) and } 24 \% \\
\text { (control) at baseline but } \\
\text { shifted to } 16 \% \text { (QG) and } \\
32 \% \text { (control) at } 12 \text {-month } \\
\text { follow-up }\end{array}$ \\
\hline
\end{tabular}




\begin{tabular}{|c|c|c|c|c|c|c|c|c|c|c|c|c|}
\hline $\begin{array}{l}\text { Stenlund } e t \text { al. } \\
\text { (2005) [123] }\end{array}$ & 95 & Sweden & $\begin{array}{l}\text { QG: } 77 \text { (3), } \\
73-92 \\
\text { C: } 78 \text { (3), } \\
73-84\end{array}$ & $66 \mathrm{M}, 29 \mathrm{~F}$ & NR & $\begin{array}{l}\text { TC and } \\
\text { medicinal } \\
\text { QG }\end{array}$ & $\begin{array}{l}12 \text { weeks, } 60 \\
\text { min QG and } \\
120 \text { min of } \\
\text { discussion on } \\
\text { various themes, } \\
\text { meeting } \\
\text { weekly }\end{array}$ & $\mathrm{N}=48$ & $\begin{array}{l}\text { Usual } \\
\text { Care } \\
(\mathrm{n}=47)\end{array}$ & \begin{tabular}{l|} 
Effect of QG \\
on physical \\
ability on \\
subjects with \\
coronary artery \\
disease
\end{tabular} & $\begin{array}{l}\text { 1) Physical } \\
\text { function } \\
\text { (Perceived } \\
\text { activity } \\
\text { level, tandem } \\
\text { standing, } \\
\text { right/left one- } \\
\text { leg stance, } \\
\text { right/left } \\
\text { co-ordination, } \\
\text { climb boxes }\end{array}$ & $\begin{array}{l}\text { Significant improvement } \\
\text { for QG compared to } \\
\text { Control group in self- } \\
\text { estimated level of physical } \\
\text { activity }(P=0.011) \text {, and } \\
\text { their performance in the } \\
\text { one-leg stance test for the } \\
\text { right leg }(P=0.029) \text {, co- } \\
\text { ordination }(P=0.021) \text { and } \\
\text { the box-climbing test for } \\
\text { right leg }(P=0.035)\end{array}$ \\
\hline $\begin{array}{l}\text { Astin } e t \text { al. } \\
\text { (2003) [124] }\end{array}$ & 128 & US & $\begin{array}{l}18-70 \\
47.7(10.6)\end{array}$ & $\begin{array}{l}\text { QG: } 1 \mathrm{M}, 63 \mathrm{~F} \\
\text { C: } 64 \mathrm{~F}\end{array}$ & NR & $\begin{array}{l}\text { Mindfulness } \\
\text { meditation } \\
\text { with QG } \\
\text { movement }\end{array}$ & $\begin{array}{l}8 \text { weeks, } 1 \mathrm{x} / \\
\text { week, } 150 \\
\text { minutes ( } 90 \text { min } \\
\text { mindfulness, } 60 \\
\text { min Qigong) } \\
\text { Data collected } \\
\text { at baseline, } \\
8 \text { weeks, } 14 \\
\text { weeks, and } 24 \\
\text { weeks }\end{array}$ & $\mathrm{N}=64$ & $\begin{array}{l}\text { Education } \\
\text { and } \\
\text { support } \\
\text { group, } \\
\mathrm{N}=64\end{array}$ & $\begin{array}{l}\text { Test benefits of } \\
\text { a mind-body } \\
\text { intervention } \\
\text { for individuals } \\
\text { with } \\
\text { fibromyalgia }\end{array}$ & $\begin{array}{l}\text { 1) } \\
\text { Fibromyalgia } \\
\text { impact (FIQ) } \\
\text { 2) Pain (SF- } \\
\text { 36) } \\
\text { 3) Depression } \\
\text { (BDI) }\end{array}$ & $\begin{array}{l}\text { No significant differences } \\
\text { between groups at } 24 \\
\text { weeks } \\
\text { Baseline vs. } 24 \text { weeks, } \\
\text { QG improvement: } \\
\text { 1) } 57.8 \pm 10.8 \text { vs. } \\
46.4 \pm 19.5, \text { p }<.01 \\
\text { 2) } 32.3 \pm 14.4 \text { vs. } \\
41.6 \pm 22.2, \text { p }<.05 \\
\text { 3) } 16.7 \pm 7.4 \text { vs. } 12.3 \pm 7.6 \text {, } \\
\text { p }<.001\end{array}$ \\
\hline \multicolumn{13}{|l|}{ Psychological } \\
\hline $\begin{array}{l}\text { Hsieh } \text { et al. } \\
\text { (2015) [77] }\end{array}$ & 66 & Taiwan & $\begin{array}{l}\text { QG: } 81.21 \\
(6.24) \\
\text { C: } 83.42 \\
(7.87)\end{array}$ & $\begin{array}{l}\text { QG: } 13 \mathrm{M} \text {, } \\
20 \mathrm{~F} \\
\text { C: } 18 \mathrm{M}, 15 \mathrm{~F}\end{array}$ & NR & LQG & $\begin{array}{l}4 \text { weeks, twice } \\
\text { a week, } 60 \text { min } \\
\text { led by LQG } \\
\text { practitioner } \\
\text { Data collected } \\
\text { at baseline } \\
\text { week } 4\end{array}$ & $\mathrm{~N}=33$ & $\mathrm{~N}=33$ & $\begin{array}{l}\text { Determine } \\
\text { psychological } \\
\text { and } \\
\text { physiological } \\
\text { effects of LQG } \\
\text { on elderly in an } \\
\text { institutionalized } \\
\text { setting }\end{array}$ & $\begin{array}{l}\text { 1) Memory } \\
\text { (MMSE) } \\
\text { 2) Mood } \\
\text { (Faces Scale) } \\
\text { 3) Depression } \\
\text { (GDS) } \\
\text { 4) Cortisol } \\
\text { levels }\end{array}$ & 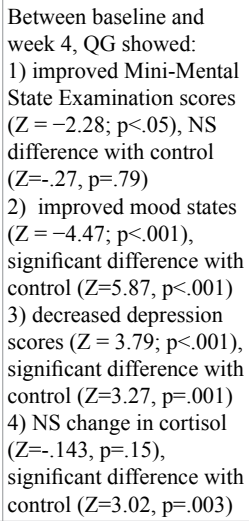 \\
\hline $\begin{array}{l}\text { Chen et al. } \\
(2013)[76]\end{array}$ & 96 & China & $\begin{array}{l}\text { QG: } 45.3 \\
(6.3), 29-58 \\
\text { C: } 44.7 \text { (9.7), } \\
35-62\end{array}$ & $96 \mathrm{~F}$ & None & $\begin{array}{l}\text { Guolin } \\
\text { (walking } \\
\text { Qigong) }\end{array}$ & $\begin{array}{l}\text { 5-6 weeks, } \\
\text { five } 40 \text {-minute } \\
\text { Qigong classes } \\
\text { Data collected } \\
\text { at baseline, } \\
\text { middle of } \\
\text { radiotherapy, } \\
\text { last week of } \\
\text { radiotherapy, } \\
1 \text { month post } \\
\text { radiotherapy, } \\
\text { and } 3 \\
\text { months post } \\
\text { radiotherapy }\end{array}$ & $\mathrm{N}=49$ & $\begin{array}{l}\text { Wait-list } \\
\text { control, } \\
\mathrm{N}=47\end{array}$ & $\begin{array}{l}\text { Examine QG } \\
\text { effects on QoL } \\
\text { on women } \\
\text { with breast } \\
\text { cancer during } \\
\text { radiotherapy }\end{array}$ & $\begin{array}{l}\text { 1) Depression } \\
\text { (CES-D) } \\
\text { 2) Fatigue } \\
\text { (BFI) } \\
\text { 3) Sleep } \\
\text { (PSQI) } \\
\text { 4) QoL } \\
\text { (FACT-G) } \\
\text { 5) Cortisol }\end{array}$ & $\begin{array}{l}\text { 1) Significant group } \\
\text { differences in depression } \\
\text { over time (F }[3,281]= \\
2.62 ; \mathrm{P}=.05) \text {. } \\
\text { QG depression score at } \\
\text { baseline vs. } 3 \text { months } \\
\text { post-radiotherapy: } \\
13.1 \pm 8.9 \text { vs. } 9.6 \pm 6.6 \\
\text { Control depression score } \\
\text { at baseline vs. } 3 \text { months } \\
\text { post-radiotherapy: } \\
12.2 \pm 9.2 \text { vs. } 11.2 \pm 9.8 \\
2-5) \text { No significant } \\
\text { differences between } Q G \\
\text { and Control groups. }\end{array}$ \\
\hline $\begin{array}{l}\text { Tsang et al. } \\
\text { (2013) [125] }\end{array}$ & 116 & HK & $\begin{array}{l}\text { QG: } 83.3 \\
(6.3) \\
\text { C: } 84.9(6.0)\end{array}$ & $\begin{array}{l}\text { QG: } 14 \mathrm{M}, \\
47 \mathrm{~F} \\
\text { C: } 15 \mathrm{M}, 40 \mathrm{~F}\end{array}$ & NR & $\begin{array}{l}\text { Yan Chai } \\
\text { Yi Jin Ten- } \\
\text { Section } \\
\text { Brocades } \\
\\
\end{array}$ & $\begin{array}{l}12 \text { weeks, two } \\
60 \text { minute } \\
\text { sessions per } \\
\text { week } \\
\text { Data collected } \\
\text { at baseline, } \\
\text { week } 6,12 \\
\text { weeks, and } 20 \\
\text { weeks }\end{array}$ & $\mathrm{N}=61$ & $\begin{array}{l}\text { Newspaper } \\
\text { reading } \\
\text { group, } \\
\mathrm{n}=55\end{array}$ & $\begin{array}{l}\text { Effectiveness } \\
\text { of QG for } \\
\text { improving } \\
\text { psychosocial, } \\
\text { cognitive, } \\
\text { physical, and } \\
\text { physiological } \\
\text { functioning } \\
\text { in frail older } \\
\text { adults }\end{array}$ & $\begin{array}{l}\text { 1) } \\
\text { Psychological } \\
\text { (GDS and } \\
\text { PBQ) } \\
\text { 2) Cognitive } \\
\text { function } \\
\text { (LOTCA-G) } \\
\text { 3) Physical } \\
\text { function } \\
\text { (Handgrip } \\
\text { strength) } \\
\text { 4) } \\
\text { Physiological } \\
\text { functioning } \\
\text { (HR, BP) }\end{array}$ & 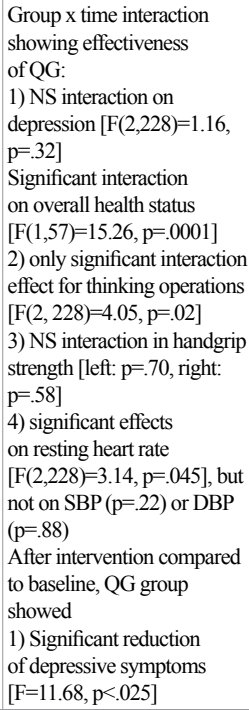 \\
\hline
\end{tabular}




\begin{tabular}{|c|c|c|c|c|c|c|c|c|c|c|c|c|}
\hline $\begin{array}{l}\text { Tsang et al. } \\
\text { (2013) [78] }\end{array}$ & 37 & HK & $\begin{array}{l}\text { QG: } 80 \text { (7) } \\
\text { C: } 81 \text { (9) }\end{array}$ & $\begin{array}{l}\text { QG: } 5 \mathrm{M}, 16 \mathrm{~F} \\
\text { C: } 7 \mathrm{M}, 10 \mathrm{~F}\end{array}$ & NR & BDJ & $\begin{array}{l}12 \text { weeks, three } \\
45 \text { min sessions } \\
\text { per week } \\
\text { Data collected } \\
\text { at baseline, } \\
\text { week } 6 \text {, week } \\
12 \text {, week } 16, \\
\text { week } 24\end{array}$ & $\mathrm{~N}=21$ & $\begin{array}{l}\text { Newspapers } \\
\text { reading, } \\
\mathrm{N}=17 \\
\\
\end{array}$ & $\begin{array}{l}\text { Examine } \\
\text { psychological, } \\
\text { physical and } \\
\text { neurophysi- } \\
\text { ological effects } \\
\text { of a QG exer- } \\
\text { cise program } \\
\text { on depressed } \\
\text { elders }\end{array}$ & $\begin{array}{l}\text { 1) Depression } \\
\text { (GDS) } \\
\text { 2) Physical (grip } \\
\text { strength) } \\
\text { 3) Neurophysi- } \\
\text { ological (cor- } \\
\text { tisol) }\end{array}$ & $\begin{array}{l}\text { 1) significant group } \mathrm{x} \\
\text { time effects on GDS } \\
{[\mathrm{F}(2,35)=5.72 ; \mathrm{p}=.007]} \\
\text { QG had higher response } \\
\text { and remission rates than } \\
\text { the comparison group } \\
\text { concerning depression } \\
(43 \% \text { vs. } 6 \%, 24 \% \text { vs. } 6 \% \text {, } \\
\text { respectively; ps }<.01) \\
\text { 2) QG: improvement } \\
\text { for right hand by } 18 \% \text {, } \\
\mathrm{p}=.034 \text {. Improvement for } \\
\text { left hand by } 26 \%, \mathrm{p}=.164 \\
\text { 3) QG: Non-significant } \\
\text { decreasing trend of } \\
\text { cortisol level by } 18.5 \%\end{array}$ \\
\hline $\begin{array}{l}\text { Chow et al. } \\
\text { (2012) [126] }\end{array}$ & 65 & HK & $\begin{array}{l}21-64 \\
\text { QG:43.79 } \\
(10.37) \\
\text { C: }: 44.66 \\
(11.86)\end{array}$ & $\begin{array}{l}\text { QG: } 12 \mathrm{M}, \\
22 \mathrm{~F} \\
\text { C: } 11 \mathrm{M}, 23 \mathrm{~F}\end{array}$ & None & $\begin{array}{l}\text { Chan Mi } \\
\text { gong }\end{array}$ & $\begin{array}{l}12 \text { weeks, } \\
\text { Weekly } 90 \text { min } \\
\text { practice with } \\
\text { instructor for } \\
\text { first } 8 \text { weeks, } \\
\text { then } 4 \text { weeks of } \\
\text { home practice } \\
\text { Data collected } \\
\text { in weeks } 1,4 \text {, } \\
8 \text {, and } 12\end{array}$ & $\mathrm{~N}=34$ & $\begin{array}{l}\text { Wait-list, } \\
\mathrm{N}=31\end{array}$ & $\begin{array}{l}\text { Investigate } \\
\text { whether QG } \\
\text { helps to reduce } \\
\text { stress and } \\
\text { anxiety }\end{array}$ & $\begin{array}{l}\text { 1) Mood } \\
\text { States (DASS- } \\
\text { 21) } \\
\text { 2) QoL } \\
\text { (ChQOL) } \\
\\
\\
\end{array}$ & $\begin{array}{l}\text { Between group } \\
\text { comparisons between } \\
\text { week } 1 \text { and week } 12, \mathrm{QG} \\
\text { vs. Control: } \\
\text { 1) significant differences } \\
\text { in overall psychological } \\
\text { wellbeing ( } \mathrm{F}[1,63]=4.26 \text {, } \\
\left.\text { p.043, } \mathrm{n}^{2}=.063\right) \\
\text { 2) significant differences } \\
\text { in } \mathrm{QoL}(\mathrm{F}[1,63]=6.04, \\
\left.\mathrm{p}=.017, \mathrm{n}^{2}=.088\right) \\
\text { Between week } 1 \text { and week } \\
12, \mathrm{QG} \text { experienced: } \\
\text { 1) significant decrease } \\
\text { in stress }(\mathrm{F}[1,63]=5.77, \\
\mathrm{p}=.019) \text { and anxiety } \\
\text { (F[1,63] }=4.72, \mathrm{p}=.034) \\
\text { 2) significant increase } \\
\text { in overall } \mathrm{Q} \text { oL } \\
(\mathrm{F}[1,63]=6.04, \mathrm{p}=.017)\end{array}$ \\
\hline $\begin{array}{l}\text { Chan et al. } \\
\text { (2011) [75] }\end{array}$ & 40 & HK & $\begin{array}{l}25-64 \\
\text { QG: } 49.65 \\
\text { (7.27) } \\
\text { C: } 48.92 \\
(8.11)\end{array}$ & $\begin{array}{l}\text { QG: } 7 \mathrm{M}, 13 \mathrm{~F} \\
\text { C: } 7 \mathrm{M}, 13 \mathrm{~F}\end{array}$ & NR & DMBI & $\begin{array}{l}4 \text { weeks, } \\
\text { weekly 90-min } \\
\text { sessions } \\
\text { Data collected } \\
\text { at baseline and } \\
4 \text { weeks }\end{array}$ & $\mathrm{N}=20$ & $\begin{array}{l}\text { Group } \\
\text { CBT, } \\
\mathrm{N}=20\end{array}$ & $\begin{array}{l}\text { Effectiveness } \\
\text { of a short-term } \\
\text { mind-body } \\
\text { intervention } \\
\text { program on } \\
\text { improving } \\
\text { depressive } \\
\text { mood }\end{array}$ & $\begin{array}{l}\text { 1) Depression } \\
\text { (BDI-II) }\end{array}$ & $\begin{array}{l}\text { 1) QG: significant } \\
\text { reduction in depressive } \\
\text { mood after treatment } \\
\text { [baseline }=14, \mathrm{SD}= \\
10.42 \text {; post-test }=6.30, \\
\mathrm{SD}=6.67 ; \mathrm{t}(19)=3.82, \\
\mathrm{p}=.001 ; \text { effect size } \\
(\text { Cohen's } d)=0.85 \text { ] } \\
\text { Extent of reduction } \\
\text { for } \mathrm{QG} \text { vs. } \mathrm{CBT} \text { was } \\
\text { significantly greater } \\
(\mathrm{p}<.05)\end{array}$ \\
\hline $\begin{array}{l}\text { Johansson et al. } \\
\text { (2011) [56] }\end{array}$ & 59 & Sweden & $50.8(12.9)$ & $51 \mathrm{~F}, 8 \mathrm{M}$ & $\begin{array}{l}\text { Average } \\
4.8 \pm 3.1 \text { years } \\
\text { of practice }\end{array}$ & $\begin{array}{l}\text { Jichu } \\
\text { Gong }\end{array}$ & $\begin{array}{l}30 \text {-min practice } \\
\text { Control: } \\
\text { listened to a } 30 \\
\text { min lecture } \\
\text { Data collected } \\
\text { at baseline and } \\
\text { after } 30 \text { min } \\
\text { exercise }\end{array}$ & $\mathrm{N}=28$ & $\begin{array}{l}\text { Lecture on } \\
\text { Chinese } \\
\text { medicine, } \\
\mathrm{N}=31\end{array}$ & $\begin{array}{l}\text { Investigate } \\
\text { acute } \\
\text { psychological } \\
\text { effects of QG } \\
\text { among regular } \\
\text { QG exercisers }\end{array}$ & $\begin{array}{l}\text { 1) Depression } \\
\text { (POMS) } \\
\text { 2) Anxiety } \\
\text { (STAI) } \\
\text { 3) Anger } \\
\text { (POMS) } \\
\text { 4) Fatigue } \\
\text { (POMS) } \\
\\
\\
\end{array}$ & 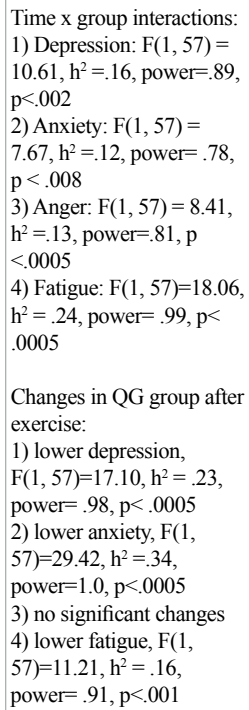 \\
\hline
\end{tabular}




\begin{tabular}{|c|c|c|c|c|c|c|c|c|c|c|c|c|}
\hline $\begin{array}{l}\text { Stenlund } e t \text { al. } \\
\text { (2009) [127] }\end{array}$ & 82 & Sweden & $\begin{array}{l}25-65 \\
\text { QG: } 43.8 \\
(9.7) \\
\text { C: } 44.7(8.6)\end{array}$ & $\begin{array}{l}\text { QG: 7M, 34F } \\
\text { C: } 7 \mathrm{M}, 34 \mathrm{~F}\end{array}$ & NR & NR & $\begin{array}{l}12 \text { weeks, } \\
\text { twice a week, } \\
1 \text { hour group } \\
\text { practice plus } \\
\text { home practice } \\
\text { Data collected } \\
\text { at baseline and } \\
12 \text { weeks }\end{array}$ & $\mathrm{N}=41$ & $\begin{array}{l}\text { Usual } \\
\text { care, } \\
\mathrm{N}=41\end{array}$ & $\begin{array}{l}\text { Evaluate } \\
\text { efficacy } \\
\text { of QG in } \\
\text { rehabilitation } \\
\text { for patients } \\
\text { with burnout }\end{array}$ & $\begin{array}{l}\text { 1) Burnout } \\
\text { (SMBQ) }\end{array}$ & $\begin{array}{l}\text { 1) At } 12 \text { weeks, NS } \\
\text { difference between } \\
\text { groups, } \mathrm{p}=.70 \\
\text { QG: significantly lower } \\
\text { median score after } \\
\text { program, } 5.8(5.0-6.0) \text { vs. } \\
5.4(4.4-5.8), \mathrm{p}<.001 \\
\text { C: significantly lower } \\
\text { median score after } \\
\text { program, } 5.8(4.8-6.2) \text { vs. } \\
5.0(4.5-5.7), \mathrm{p}<.001\end{array}$ \\
\hline $\begin{array}{l}\text { Griffith et al. } \\
\text { (2008) [128] }\end{array}$ & 37 & US & $\begin{array}{l}\text { QG: } 52(9) \\
\text { C: } 50(10)\end{array}$ & $\begin{array}{l}\text { QG: } 12 \mathrm{~F}, 4 \mathrm{M} \\
\text { C: } 17 \mathrm{~F}, 5 \mathrm{M}\end{array}$ & None & BDJ & $\begin{array}{l}6 \text { weeks, twice } \\
\text { a week, } 1 \text { hour } \\
\text { class } \\
\text { Asked to } \\
\text { practice for } 30 \\
\text { min on non- } \\
\text { class days } \\
\text { Data collected } \\
\text { at baseline and } \\
6 \text { weeks }\end{array}$ & $\mathrm{N}=16$ & $\begin{array}{l}\text { Wait-list, } \\
\text { control, } \\
\mathrm{N}=21\end{array}$ & $\begin{array}{l}\text { Investigate } \\
\text { effectiveness of } \\
\text { QG in reducing } \\
\text { stress in } \\
\text { hospital staff }\end{array}$ & $\begin{array}{l}\text { 1) Stress } \\
\text { (PSS) } \\
\text { 2) QoL (SF- } \\
\text { 36) } \\
\text { 3) Pain (VAS) }\end{array}$ & $\begin{array}{l}\text { 1) Significant difference } \\
\text { in reduction of perceived } \\
\text { stress over time between } \\
\text { QG and Control ( } \mathrm{t}=- \\
.2458, \mathrm{p}=.02) \\
\text { QG: decreased mean=- } \\
4.5 \pm 6.6 \\
\text { 2) Significant difference } \\
\text { in social functioning } \\
\text { score over time between } \\
\mathrm{QG} \text { and control ( } \mathrm{t}=2.035 \text {, } \\
\mathrm{p}=.05) \\
\text { 3) NS difference in } \\
\text { reduction of pain over } \\
\text { time between } \mathrm{QG} \text { and } \\
\text { Control }(\mathrm{t}=-1.097, \mathrm{p}=.28)\end{array}$ \\
\hline $\begin{array}{l}\text { Haak and Scott } \\
\text { (2008) [129] }\end{array}$ & 57 & Sweden & $\begin{array}{l}27-73 \\
\text { QG: } 54.0 \\
(9.4) \\
\text { C: } 53.4(8.0)\end{array}$ & $57 \mathrm{~F}$ & $\begin{array}{l}85 \% \text { have } \\
\text { used some } \\
\text { form of CAM }\end{array}$ & $\begin{array}{l}\text { Lotus } \\
\text { method } \\
\text { (He Hua } \\
\text { QG) } \\
\text { EQT }\end{array}$ & $\begin{array}{l}7 \text { weeks, } 9 \\
\text { group session } \\
\text { (Total } 11.5 \\
\text { hours) } \\
\text { Encouraged to } \\
\text { practice twice } \\
\text { a day for } 20 \\
\text { minutes } \\
\text { Received EQT } \\
\text { twice during } \\
\text { intervention } \\
\text { Data collected } \\
\text { at baseline, } 7 \\
\text { weeks, and } 4 \\
\text { months }\end{array}$ & $\mathrm{N}=29$ & $\begin{array}{l}\text { Wait-list, } \\
\mathrm{N}=28 \\
\text { At } 4 \text { month } \\
\text { follow up, } \\
\text { control had } \\
\text { received in- } \\
\text { tervention }\end{array}$ & $\begin{array}{l}\text { Evaluate the } \\
\text { effects of QG } \\
\text { on pain, quality } \\
\text { of sleep, } \\
\text { psychological } \\
\text { health, work } \\
\text { status, and use } \\
\text { of medication }\end{array}$ & $\begin{array}{l}\text { 1) Anxiety } \\
\text { (STAI) } \\
\text { 2) Depression } \\
\text { (BDI) } \\
\text { 3) QoL } \\
\text { (WHOQOL- } \\
\text { BREF) } \\
\text { 4) Pain (VNS) }\end{array}$ & $\begin{array}{l}\text { Group } \mathrm{x} \text { time differences } \\
\text { between intervention and } \\
\text { control at baseline to } 7 \\
\text { weeks } \\
\text { 1) anxiety: } \mathrm{F}(1,55)=5.81 \text {, } \\
\mathrm{p}<.05 \\
\text { 2) depression: } \\
\mathrm{F}(1,55)=6.44, \mathrm{p}<.01 \\
\text { 3) quality of life: } \\
\mathrm{F}(1,55)=4.03, \mathrm{p}<.05 \\
\text { 4)intensity of pain: } \\
\mathrm{F}(1,55)=5.34, \mathrm{p}<.05 \\
\text { Time interaction of } \\
\text { combined (N=57) group } \\
\text { changes after intervention } \\
\text { (baseline, } 7 \text { weeks, } 4 \\
\text { months) } \\
\text { 1) decreased anxiety: } \mathrm{F} \\
\text { time }=4.90, \mathrm{p}<.01 \\
\text { 2) decreased depressive } \\
\text { symptoms: } \mathrm{F} \text { time }=6.80, \\
\mathrm{p}<.01 \\
\text { 3) increased quality of } \\
\text { life: } \mathrm{F} \text { time }=6.24, \mathrm{p}<.01 \\
\text { 4) decreased intensity of } \\
\text { pain: } \mathrm{F} \text { time }=7.88, \mathrm{p}<.001\end{array}$ \\
\hline $\begin{array}{l}\text { Tsang et al. } \\
\text { (2006) [130] }\end{array}$ & 82 & HK & $\begin{array}{l}\text { QG: } 82.11 \\
(7.19) \\
\text { C: } 82.74 \\
(6.83)\end{array}$ & $\begin{array}{l}\text { QG: } 10 \mathrm{M}, \\
28 \mathrm{~F} \\
\text { C: } 6 \mathrm{M}, 28 \mathrm{~F}\end{array}$ & NR & BDJ & $\begin{array}{l}16 \text { weeks, } 3 \\
\text { days a week, } \\
30-45 \text { min } \\
\text { each } \\
\text { Data collected } \\
\text { at baseline, } 8 \\
\text { weeks, and } 16 \\
\text { weeks }\end{array}$ & $\mathrm{N}=48$ & $\begin{array}{l}\text { Newspa- } \\
\text { per Read- } \\
\text { ing group } \\
\text { of similar } \\
\text { intensity, } \\
\mathrm{N}=34\end{array}$ & $\begin{array}{l}\text { Understand the } \\
\text { psycho-social } \\
\text { effects of QG } \\
\text { on elderly } \\
\text { persons with } \\
\text { depression }\end{array}$ & $\begin{array}{l}\text { 1) Depression } \\
\text { (GDS) } \\
\text { 2) Self- } \\
\text { efficacy } \\
\text { (CGSS) }\end{array}$ & $\begin{array}{l}\text { Group } \mathrm{x} \text { time interaction } \\
\text { among the two groups at } \\
\text { five different time points: } \\
\text { 1) Depression }[\mathrm{F}(4, \\
77)=2.619, \mathrm{p}=0.041] \text {, } \\
\text { 2) Self-efficacy }[\mathrm{F}(4, \\
77)=11.693, \mathrm{p}<0.001] \\
\text { After practicing } \mathrm{QG} \text { : } \\
\text { 1) improvement in } \\
\text { depression, significance } \\
\text { not reported } \\
\text { 2) improvement in self- } \\
\text { efficacy, significance not } \\
\text { reported }\end{array}$ \\
\hline
\end{tabular}




\begin{tabular}{|c|c|c|c|c|c|c|c|c|c|c|c|c|}
\hline $\begin{array}{l}\text { Tsang et al. } \\
\text { (2003) [131] }\end{array}$ & 50 & HK & $\begin{array}{l}\text { G: } 72.9(9.5) \\
\text { C: } 76.3(8.4)\end{array}$ & $\begin{array}{l}\text { QG: } 9 \mathrm{M}, 15 \mathrm{~F} \\
\text { C: } 17 \mathrm{M}, 9 \mathrm{~F}\end{array}$ & NR & BDJ & $\begin{array}{l}12 \text { weeks, } 2 \\
\text { days/week, } 60 \\
\text { min each } \\
\text { Data collected } \\
\text { at baseline, } \\
\text { midway, and } \\
\text { post-program }\end{array}$ & $\mathrm{N}=24$ & $\begin{array}{l}\text { Basic } \\
\text { Reha- } \\
\text { bilitation } \\
\text { activities, } \\
\mathrm{N}=26\end{array}$ & $\begin{array}{l}\text { Assess if BDJ } \\
\text { improves bio- } \\
\text { psychosocial } \\
\text { health of par- } \\
\text { ticipants }\end{array}$ & $\begin{array}{l}\text { 1) Depression } \\
\text { (GDS) } \\
\text { 2) Perceived } \\
\text { benefit (PBQ) }\end{array}$ & $\begin{array}{l}\text { 1) repeated measures } \\
\text { ANOVA of two groups } \\
\text { is not significant }[\mathrm{F}(2, \\
39)=2.032, \mathrm{p}=.145] \\
\text { 2) QG group has } \\
\text { perceived improvement } \\
\text { in physical health } \\
{[19.36 \pm 2.79, \mathrm{t}(21)=7.34,} \\
\mathrm{p}<.001], \text { overall } \mathrm{ADL} \\
{[14.75 \pm 2.12, \mathrm{t}(7)=3.67,} \\
\mathrm{p}=.008], \text { psychological } \\
\text { health }[26.73 \pm 2.91 \\
\mathrm{t}(21)=9.22, \mathrm{p}<.001] \\
\text { social relationship } \\
{[11.05 \pm 1.94, \mathrm{t}(21)=4.95,} \\
\mathrm{p}<.001], \text { and health in } \\
\text { general }[7.50 \pm 1.06, \\
\mathrm{t}(21)=6.65, \mathrm{p}<.001]\end{array}$ \\
\hline \multicolumn{13}{|l|}{ Quality of Life } \\
\hline $\begin{array}{l}\text { Oh et al. (2014) } \\
\text { [72] }\end{array}$ & 27 & $\mathrm{AU}$ & $\begin{array}{l}\text { QG: } 56.9 \\
(12.1) \\
\text { C: } 57.8(10.8)\end{array}$ & $27 \mathrm{~F}$ & None & MQ & $\begin{array}{l}10 \text { weeks, } \\
\text { once a week, } \\
60 \text { min group } \\
\text { supervised } \\
\text { class } \\
\text { Encouraged } \\
\text { to practice at } \\
\text { home for } 30 \\
\text { min each day } \\
\text { Data collected } \\
\text { at baseline, } \\
\text { week } 5 \text {, and } \\
\text { week } 10\end{array}$ & $\mathrm{~N}=14$ & $\begin{array}{l}\text { Medita- } \\
\text { tion, } \mathrm{N}=13\end{array}$ & $\begin{array}{l}\text { Examine the } \\
\text { feasibility, } \\
\text { safety, and } \\
\text { effects of MQ } \\
\text { in improving } \\
\text { QoL in women } \\
\text { with metastatic } \\
\text { breast cancer }\end{array}$ & \begin{tabular}{l|} 
1) QoL \\
(FACT-B) \\
2) Fatigue \\
(FACT-F) \\
3) Perceived \\
stress (PSS) \\
4) Neuropathic \\
symptoms \\
(FACT/ GOG- \\
NTX) \\
\end{tabular} & $\begin{array}{l}\text { At } 10 \text { weeks: } \\
\text { 1) No significant } \\
\text { differences between } \\
\text { groups ( } \mathrm{p}=0.84 \text { ) } \\
\text { 2) No significant } \\
\text { differences between } \\
\text { groups ( } \mathrm{p}=0.71 \text { ) } \\
\text { 3) No significant } \\
\text { differences between } \\
\text { groups ( }=0.52) \\
\text { 4) Significant group } \\
\text { difference }(0=0.014), \mathrm{QG} \\
\text { improved while control } \\
\text { deteriorated }\end{array}$ \\
\hline $\begin{array}{l}\text { Lin } e \text { tal. } \\
\text { (2012) [132]\# }\end{array}$ & 60 & China & $\begin{array}{l}50-85 \\
\text { QG: } 66.47 \\
(8.26) \\
\text { C: } 64.9(8.87)\end{array}$ & $\begin{array}{l}\text { QG: } 24 \mathrm{M}, 6 \mathrm{~F} \\
\text { C: } 22 \mathrm{M}, 8 \mathrm{~F} \\
\end{array}$ & None & BDJ & $\begin{array}{l}23 \text { weeks } \\
\text { Data } \\
\text { collected at } \\
\text { baseline, mid- } \\
\text { intervention, } \\
\text { and } 23 \text { weeks }\end{array}$ & $\mathrm{N}=30$ & $\mathrm{~N}=30$ & $\begin{array}{l}\text { Explore the } \\
\text { effect of BDJ } \\
\text { exercise on } \\
\text { quality of life } \\
\text { of patients } \\
\text { after Coronary } \\
\text { artery bypass } \\
\text { grafting }\end{array}$ & $\begin{array}{l}\text { 1) QoL } \\
\text { (QOLS) } \\
\text { 2) Functional } \\
\text { status (SAQ) }\end{array}$ & $\begin{array}{l}\text { QG compared to Control } \\
\text { group } \\
\text { 1) improvement of } \mathrm{Q} \text { L } \\
\mathrm{p}<.05 \\
\text { 2) improvement of } \\
\text { functional status, } \mathrm{p}<.05\end{array}$ \\
\hline $\begin{array}{l}\text { Oh et al. (2010) } \\
\text { [71] }\end{array}$ & 162 & Australia & $\begin{array}{l}31-86 \\
\text { QG: } 60.1 \\
(11.7) \\
\text { C: } 59.9(11.3)\end{array}$ & $\begin{array}{l}\text { QG: } 48 \mathrm{~F} \text {, } \\
31 \mathrm{M} \\
\text { C: } 45 \mathrm{~F}, 38 \mathrm{M}\end{array}$ & None & MQ & $\begin{array}{l}10 \text { weeks, two } \\
\text { supervised } 90- \\
\text { min sessions } \\
\text { per week. } \\
\text { Participants } \\
\text { encouraged } \\
\text { practice at } \\
\text { home every } \\
\text { day for at least } \\
39 \text { min } \\
\text { Data collected }\end{array}$ & $\mathrm{N}=79$ & $\begin{array}{l}\text { Usual } \\
\text { care, } \\
\mathrm{N}=83\end{array}$ & $\begin{array}{l}\text { Evaluate use of } \\
\text { MQ compared } \\
\text { with usual care } \\
\text { to improve } \\
\text { quality of } \\
\text { life of cancer } \\
\text { patients }\end{array}$ & $\begin{array}{l}\text { 1) QoL } \\
\text { (FACT-G) } \\
\text { 2) Fatigue } \\
\text { (FACT-F) } \\
\text { 3) Mood } \\
\text { (POMS) } \\
\text { 4) } \\
\text { Inflammation } \\
\text { (CRP) }\end{array}$ & $\begin{array}{l}\text { At } 10 \text { weeks, QG } \\
\text { compared to } \mathrm{C} \\
\text { (controlling for gender, } \\
\text { age, status of cancer } \\
\text { treatment, week } 0 \text { baseline } \\
\text { scores, and intervention } \\
\text { status) } \\
\text { 1) improved overall QoL, } \\
\text { mean difference }=9.00 \text { (CI, } \\
5.62 \text { to } 12.36 \text { ), }<<.001 \\
\text { 2) improved fatigue score, } \\
\text { mean difference }=5.70 \text { (CI, } \\
3.32 \text { to } 8.09 \text { ), } p<.001 \\
\text { 3) improved total } \\
\text { mood status, mean } \\
\text { difference }=-10.64 \text { (CI, } \\
-19.81 \text { to }-1.47), p=.02 \\
\text { 4) less inflammation, } \\
\text { mean difference }=-23.17 \\
\text { (CI, }-37.08 \text { to }-9.26 \text { ), } \\
\text { p=.04 }\end{array}$ \\
\hline $\begin{array}{l}\text { Oh et al. (2008) } \\
\text { [73] }\end{array}$ & 30 & Australia & 54 (9), 35-75 & $\begin{array}{l}\text { QG: } 3 \mathrm{M}, 12 \mathrm{~F} \\
\mathrm{C}: 3 \mathrm{M}, 12 \mathrm{~F}\end{array}$ & None & MQ & $\begin{array}{l}8 \text { weeks, once } \\
\text { or twice a } \\
\text { week, } 90 \mathrm{mn} \\
\text { class }(15 \mathrm{~min} \\
\text { discussion, } 30 \\
\text { min stretching } \\
\text { and movement, } \\
15 \text { min seated } \\
\text { movement, } 30 \\
\text { min breathing) } \\
\text { Recommended } \\
\text { practice at } \\
\text { home very day } \\
\text { for at least } 1 \\
\text { hour } \\
\text { Data collected } \\
\text { at baseline and } \\
\text { week } 8\end{array}$ & $\mathrm{~N}=15$ & $\begin{array}{l}\text { Usual } \\
\text { care, } \\
\mathrm{N}=15\end{array}$ & $\begin{array}{l}\text { Examine } \\
\text { impact of MQ } \\
\text { for improving } \\
\text { QoL, } \\
\text { symptoms, side } \\
\text { effects, and } \\
\text { longevity }\end{array}$ & $\begin{array}{l}\text { 1) QoL } \\
\text { (ERORTC } \\
\text { QLQ-C 30) } \\
\text { 2) Symptoms } \\
\text { (ERORTC } \\
\text { QLQ-C 30) } \\
\text { 3) } \\
\text { inflammation } \\
\text { (CRP) }\end{array}$ & $\begin{array}{l}\text { No significant differences } \\
\text { between groups due to } \\
\text { small sample size } \\
\text { For QG group changes } \\
\text { from baseline to week } 8 \\
\text { 1) improvement in QoL } \\
\text { (10.4, } p=.005 \text { ) } \\
\text { 2) No significant changes } \\
\text { in fatigue, nausea, pain, } \\
\text { dyspnea, insomnia, } \\
\text { appetite, constipation, or } \\
\text { diarrhea ps }>.05 \\
\text { 3) NS increase in CRP } \\
\text { score }+1.7\end{array}$ \\
\hline
\end{tabular}




\begin{tabular}{|c|c|c|c|c|c|c|c|c|c|c|c|c|}
\hline $\begin{array}{l}\text { Wang et al. } \\
\text { (2007) [60] }\end{array}$ & 200 & China & $\begin{array}{l}\text { Male: } 61-65 \\
\text { QG: } 63(2.7) \\
\text { C: } 62.4(2.6) \\
\text { Female: } 56-60 \\
\text { QG: } 57.8 \\
(2.6) \\
\text { C: } 56.9(3.0)\end{array}$ & $\begin{array}{l}\text { QG: 60M, } \\
\text { 60F } \\
\text { C: } 40 \mathrm{M}, 40 \mathrm{~F} \\
\end{array}$ & None & BDJ & $\begin{array}{l}6 \text { months, } 1 \\
\text { hour every day } \\
\text { Data collected } \\
\text { at baseline and } \\
6 \text { months }\end{array}$ & $\mathrm{N}=120$ & $\mathrm{~N}=80$ & $\begin{array}{l}\text { explore the } \\
\text { effect of BDJ } \\
\text { exercise on } \\
\text { quality of } \\
\text { life of senior } \\
\text { people. }\end{array}$ & $\begin{array}{l}\text { 1) QoL (SF- } \\
\text { 36) }\end{array}$ & $\begin{array}{l}\text { Outcome were reported } \\
\text { based on gender } \\
\text { After } 6 \text { months, QG vs. } \\
\text { control groups: } \\
\text { 1) Male: } \\
\text { SF- } 36 \text { total: } 78.4 \pm 14 \text { vs. } \\
67.7 \pm 10, \text { p }<.05 \\
\text { Female: } \\
\text { SF- } 36 \text { total: } 81.9 \pm 13 \text { vs. } \\
76.1 \pm 12, \mathrm{p}<.05\end{array}$ \\
\hline \multicolumn{13}{|l|}{ Sleep } \\
\hline $\begin{array}{l}\text { Liao et al. } \\
\text { (2015) [133] }\end{array}$ & 131 & China & $\begin{array}{l}\text { QG: } 31.1 \\
(10.46) \\
\text { C: } 31.6 \\
(10.74)\end{array}$ & $\begin{array}{l}\text { QG: } 10 \mathrm{M} \text {, } \\
52 \mathrm{~F} \\
\mathrm{C}: 23 \mathrm{M}, 44 \mathrm{~F}\end{array}$ & NR & BDJ & $\begin{array}{l}6 \text { weeks, } \\
\text { exercised } 30 \\
\text { minutes } 2 x \\
\text { a day } \\
\text { Data collected } \\
\text { at baseline, } \\
\text { week } 4 \text {, week } \\
6 \text {, week 12, and } \\
\text { week } 18\end{array}$ & $\mathrm{~N}=64$ & $\mathrm{~N}=67$ & $\begin{array}{l}\text { Observe effect } \\
\text { of BDJ on } \\
\text { fatigue }\end{array}$ & $\begin{array}{l}\text { 1) Fatigue } \\
\text { (FSAS) }\end{array}$ & $\begin{array}{l}\text { From baseline to end of } \\
\text { week } 18 \\
\text { 1) NS group } x \text { time } \\
\text { interaction, } p=.66 \\
\text { Significant difference over } \\
\text { time: } F=34.855, p<.001 \\
\text { Significant difference } \\
\text { between groups: } \\
F=27.375, p<.001 \\
\text { QG improved between } \\
\text { baseline and } 18^{\text {th }} \\
\text { week: } 41.50 \pm 12.36 \text { vs. } \\
12.28 \pm 10.46\end{array}$ \\
\hline $\begin{array}{l}\text { Larkey et al. } \\
\text { (2014) [134] }\end{array}$ & 87 & US & $\begin{array}{l}40-75 \\
\text { QG: } 57.7 \\
(8.94) \\
\text { C: } 59.8(8.93)\end{array}$ & $87 \mathrm{~F}$ & None & QG/ TCE & $\begin{array}{l}12 \text { weeks, } 60 \\
\text { min sessions } \\
\text { meeting } 2 x \text { a } \\
\text { week for the } \\
\text { first } 2 \text { weeks } \\
\text { and then once } \\
\text { a week for the } \\
\text { remainder } \\
\text { Asked to } \\
\text { practice at } \\
\text { home at least } \\
30 \text { min a day, } 5 \\
\text { days per week } \\
\text { Data collected } \\
\text { at baseline, } \\
\text { post- } \\
\text { intervention, } 3 \\
\text { months later }\end{array}$ & $\mathrm{N}=45$ & $\begin{array}{l}\text { Sham } \\
\text { Qigong } \\
\mathrm{N}=42\end{array}$ & $\begin{array}{l}\text { Compare QG/ } \\
\text { TCE with SQG } \\
\text { on fatigue } \\
\text { and other } \\
\text { symptoms } \\
\text { among } \\
\text { breast cancer } \\
\text { survivors }\end{array}$ & $\begin{array}{l}\text { 1) Fatigue } \\
\text { (FSI) } \\
\text { 2) Depression } \\
\text { (BDI) } \\
\text { 3) Sleep } \\
\text { (PSQI) }\end{array}$ & 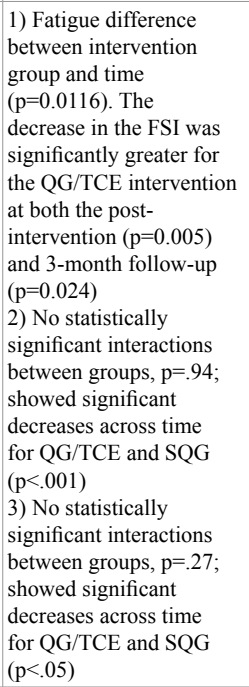 \\
\hline $\begin{array}{l}\text { Li and Wang } \\
\text { (2014) [135]\# }\end{array}$ & 40 & China & $\begin{array}{l}\text { QG: } 53.60 \text {, } \\
41-69 \\
\text { C: } 51.4 \text { (9.2), } \\
39-70\end{array}$ & $\begin{array}{l}\text { QG: } 11 \mathrm{M}, 9 \mathrm{~F} \\
\text { C: } 8 \mathrm{M}, 12 \mathrm{~F}\end{array}$ & none & BDJ & $\begin{array}{l}4 \text { weeks, } \\
\text { practice once } \\
\text { a day, } 30 \text { min } \\
\text { each } \\
\text { Data collected } \\
\text { at baseline and } \\
4 \text { weeks }\end{array}$ & $\mathrm{N}=20$ & $\mathrm{~N}=20$ & $\begin{array}{l}\text { Explore the } \\
\text { effect of BDJ } \\
\text { exercise on } \\
\text { insomnia } \\
\text { among type } 2 \\
\text { diabetes }\end{array}$ & $\begin{array}{l}\text { 1) Sleep } \\
\text { (PSQI) }\end{array}$ & $\begin{array}{l}\text { 1) After } 1 \text { month, NS } \\
\text { difference between QG } \\
\text { and control groups, } \\
\text { however there is a trend } \\
\text { of improvement from } \\
\text { baseline to } 1 \text { month for } \\
\text { QG group ( } 8.43 \pm 4.48 \text { vs. } \\
9.03 \pm 4.61 \text { ) }\end{array}$ \\
\hline $\begin{array}{l}\text { Chan } \text { et al. } \\
\text { (2012) [136] }\end{array}$ & 50 & HK & $\begin{array}{l}28-62 \\
\text { QG: } 47.06 \\
\text { (9.54) } \\
\text { CBT: } 47.39 \\
\text { (6.63) } \\
\text { WL: } 45.44 \\
(8.25)\end{array}$ & $\begin{array}{l}\text { QG: } 2 \mathrm{M}, 15 \mathrm{~F} \\
\text { CBT: } 5 \mathrm{M}, \\
13 \mathrm{~F} \\
\text { C: } 4 \mathrm{M}, 12 \mathrm{~F}\end{array}$ & NR & DMBI & $\begin{array}{l}10 \text { weeks, one } \\
\text { weekly } 90 \text { min } \\
\text { sessions for } \\
\text { either DMBI or } \\
\text { CBT } \\
\text { Data collected } \\
\text { at baseline and } \\
10 \text { weeks }\end{array}$ & $\begin{array}{l}\text { Two groups: } \\
\text { 1) } \mathrm{QG} \text {, } \\
\mathrm{N}=17 \\
\text { 2) } \mathrm{CBT} \text {, } \\
\mathrm{N}=18\end{array}$ & $\begin{array}{l}\text { Wait-list, } \\
\mathrm{N}=16\end{array}$ & \begin{tabular}{l|} 
Compare the \\
effect of DMBI \\
vs. CBT on \\
improving \\
sleep problems \\
of patients with \\
depression
\end{tabular} & $\begin{array}{l}\text { 1) sleep items } \\
\text { (HRSD) } \\
\text { 2) Total sleep } \\
\text { time (hours) } \\
\text { 3) Sleep onset } \\
\text { latency (min) } \\
\text { 4) Wake time } \\
\text { after sleep } \\
\text { onset (min) }\end{array}$ & $\begin{array}{l}\text { For QG group, mean } \\
\text { difference between } \\
\text { baseline and } 10 \text { weeks: } \\
\text { 1) }-1.50(1.51), \mathrm{p}<.01 \\
\text { 2) } 0.79(1.64), \mathrm{p}=.03 \\
\text { 3) }-9.81(21.25), \mathrm{p}=.04 \\
\text { 4) }-12.10(26.34), \mathrm{p}=.09 \\
\text { For CBT and Control, no } \\
\text { significant differences in } \\
\text { pre- and post- treatment } \\
\text { testing of sleep }\end{array}$ \\
\hline $\begin{array}{l}\text { Chen } \text { et al. } \\
\text { (2012) [137] }\end{array}$ & 56 & Taiwan & $\begin{array}{l}71.75(8.13) \\
\text { QG: } 70.48 \\
(7.90) \\
\text { C: } 72.96 \\
(8.30)\end{array}$ & $\begin{array}{l}\text { QG: 17F, } \\
10 \mathrm{M} \\
\text { C: } 19 \mathrm{~F}, 9 \mathrm{M}\end{array}$ & $\begin{array}{l}\text { None within } \\
6 \text { months }\end{array}$ & BDJ & $\begin{array}{l}12 \text { weeks, } \\
30 \text {-min home- } \\
\text { based exercise, } \\
\text { thrice a week. } \\
\text { Data collected } \\
\text { at baseline, } \\
\text { week } 4 \text {, week } \\
8 \text {, and week } 12\end{array}$ & $\mathrm{~N}=28$ & $\mathrm{~N}=28$ & $\begin{array}{l}\text { Explore } \\
\text { effectiveness } \\
\text { of BDJ on } \\
\text { sleep quality } \\
\text { in Taiwanese } \\
\text { elderly }\end{array}$ & $\begin{array}{l}\text { 1) Sleep } \\
\text { quality (PSQI) }\end{array}$ & $\begin{array}{l}\text { 1) QG: significant } \\
\text { improvement in their } \\
\text { overall sleep quality ( } \mathrm{F}= \\
26.04, \mathrm{p}<.001) \\
\text { After } 12 \text { weeks, mean } \\
\text { scores of sleep quality } \\
\text { were significantly } \\
\text { improved in the exercise } \\
\text { group over the control } \\
\text { group in overall sleep } \\
\text { quality }(\beta=-5.10, \mathrm{p}<.001)\end{array}$ \\
\hline
\end{tabular}


Qigong on balance $(n=3)$, cognitive function $(n=1)$, diabetes $(n=1)$, pain $(n=8)$, physical function/fitness $(n=13)$, psychological $(n=12)$, quality of life $(n=5)$, and sleep $(n=5)$. Twenty-six articles examined the effects of Qigong among individuals of East Asian descent. Regarding type of Qigong, Baduanjin (BDJ) was the most common and was cited in 17 studies. Four articles, one in conjunction with internal Qigong, examined the effects of EQT. Out of these articles, Chen et al. [55] found that the individual healer significantly influences the health outcome from EQT, which was tested through using randomized groups with two healers with individualized styles and a control group. Seven articles did not specify the type of Qigong practiced in the study.

Study design widely varied, as active intervention time period ranged from 30 minutes [56] to 6 months [57-63]. Twenty-nine studies used wait-list or usual care controls. Some studies tested multiple forms of intervention; eighteen studies used some form of active control, and six studies used more than two randomized groups. Previous experience with Qigong or other mind-body therapies was not consistently reported, and only one article reported the measurement and association of belief in CAM as an effective treatment and the tested health outcome [55].

Regarding balance, Liu et al. [64] and Yang et al. [63] found that Qigong improved balance among older adults, while Wenneberg et al. [65] found no significant group differences among adults of all ages with muscular dystrophy. Oh et al. [66] found that the medical Qigong intervention significantly improved cognitive function as measured by two instruments (EORTC-CF and FACT-Cog). Regarding diabetesrelated health, Wei and $\mathrm{Wu}$ [67] reported significantly better status among the Qigong intervention group versus the control group. Six out of eight studies examining pain levels after a Qigong intervention found a significant decrease in pain. Lansinger et al. [68] found no difference between a Qigong group and an exercise therapy control, and both groups showed improvement regarding pain. von Trott et al. [69] similarly found no significant difference in pain level between three randomized groups: Qigong, exercise therapy, and wait-list control. All these studies used a visual analogue scale to examine pain severity.

Improvements in physical function were found in eleven out of thirteen studies, and the measurements and types of Qigong practiced in these thirteen studies greatly varied. Notably, a few studies found significant improvements in Qigong groups versus other forms of exercise. Chan et al. [70] found that a Tai Chi Qigong group showed the greatest significant improvements in lung function and the 6-Minute Walk Test compared to both an exercise group and a control group. Xiao and Zhuang [62] found in a comparison of BDJ and walking exercise, there was a significant group by time interaction regarding functioning mobility, with greater improvements in the BDJ group.

Regarding psychological outcomes, twelve out of twelve studies found that psychological symptoms, ranging from mood to depression to burnout, significantly decreased after a Qigong intervention. For quality of life, three out of five studies showed significant improvement among Qigong group participants. After controlling for gender, age, status of cancer treatment, baseline scores, and intervention status, Oh et al. [71] found that the Qigong group had a greatly improved quality of life compared to a usual care group. On the other hand, Oh et al. [72] found no significant differences in quality of life between a medical Qigong group and a meditation group. Both studies which did not find significant differences had relatively smaller sample sizes of 30 individuals or less [72,73]. Lastly, four out of five studies showed significant improvement of sleep or fatigue among Qigong group participants, and all studies showed at least a trend of improvement after a Qigong intervention.

\section{Discussion}

Our global literature review regarding Qigong practice among older adults shows that there is significant and expanding evidence concerning the efficacy of Qigong at improving health outcomes. However, there is limited knowledge about the prevalence and characteristics of individuals who practice Qigong both in China and worldwide. Research has instead focused on clinical trials to determine the health outcomes of a Qigong intervention. Trial research has found that Qigong practice may improve certain conditions, especially those that are chronic like musculoskeletal disorders and psychological distress. Type of Qigong and length of practice may influence results. However, many limitations exist, especially concerning study design.

\section{Prevalence and practitioner characteristics}

From our findings, there is very little information about the prevalence of Qigong practice by country and internationally, as well as limited information about practitioner characteristics. In the U.S., data are mostly drawn from the National Health Interview Survey, and most research articles include estimates for an aggregated adult category and/or aggregated mind-body therapy category. However, based on these figures, Qigong is not a popular form of exercise for U.S. adults, with most estimates under $0.5 \%$. The only non-U.S. sample regarding prevalence is from Singapore, where $3.5 \%$ of older adults practice Qigong. Regarding Qigong practitioner characteristics, there is some evidence that Qigong practitioners have better health $[8,40,41,74]$ and lower medical costs [10] than non-practitioners, though it is difficult to compare across samples and not all articles included comparison groups for analysis. It is also unknown if these figures are representative of Qigong practitioners and therefore, generalizable.

Notably, data about Qigong prevalence and practitioner characteristics in China is missing. A few authors have estimated that there are 65 or 70 million Qigong practitioners in China [5,6], but how they ascertained these figures is unknown. Various Chinese political movements have influenced the practice and research of Qigong due to its possible association with religious or "subversive" activities [4], which has impacted the kind of research conducted on Qigong from China. To understand the relevance of Qigong globally, research should work to provide estimates on the number of practitioners, with special attention paid to the type of Qigong and other demographic and health related data. Population and longitudinal studies are needed to understand the practice of Qigong among community-dwelling older adults, why they practice, and the physiological, physical, and psychological health effects of this practice.

\section{Research trials}

Given the wide variety of study designs, it is beyond the ability of this review paper to confirm the findings of the research trials; however, there are a few emerging themes regarding type of Qigong, overall health improvements due to Qigong practice, comparisons to other forms of intervention or usual care, and practice length.

\section{Type of Qigong}

From the available data, it appears that there are differences in health outcome depending on the type of Qigong practiced, though it is difficult to make any concrete conclusions due to variability in study design. Regarding EQT, researchers mostly found non-significant 
findings, possibly due to small sample size. However, Chen et al. [55] found that the individual healer and their technique impacted the effect of EQT on pain, as there was no difference between one of the healers and the sham treatment but lessened pain from the other healer. Regarding internal Qigong, our findings indicated that BDJ is the most common form for testing Qigong health outcomes, used in 18 out of the 52 trial studies which reported type of Qigong. However, our review was unable to find sufficient evidence that one form of internal Qigong is more effective than another for any specific condition. For example, Laughing Qigong, Guolin Qigong, Dejian Mind-Body Qigong, and Baduanjin were all found to decrease depressive symptoms over time [75-78].

Even in studies where results are not significant, it is unclear if it is an issue in study design or the ineffectiveness of the type of Qigong. For example, Wenneberg et al. [65] did not find any group differences of patients with muscular dystrophy between an intervention and wait-list control group. However, the authors did not report the type of Qigong used, and it is unknown if another form of Qigong would have had significantly different effects on health outcomes. According to Kemp ([2], there are many forms of Qigong that can be used for a variety of conditions and physical function levels. Amano et al. [79] did not find any significant changes in physical function when comparing outcomes of a Qigong meditation group and a Tai Chi group, but the sample size was only 21 individuals. To our knowledge, there are few RCTs which examine the effectiveness of one form of Qigong to another, and further research is needed to more rigorously examine the best form of Qigong for a specific health outcome.

\section{Health outcomes}

Qigong interventions have been shown to significantly improve certain health outcomes, especially in regard to pain and psychological outcomes. Most studies which examined pain used a visual analogue scale, which is thought to be a highly reliable measurement [80]. Lynch et al. [81] found clinically significant improvements among individuals with fibromyalgia in pain between baseline and a six-month follow up in a Chaoyi Fanhuan Qigong intervention. According to the authors, Chaoyi Fanhuan Qigong is a gentle form that does not involve aerobic activities, but rather focuses on mindfulness, which some research has shown to be effective in reducing pain [82]. Regarding psychological outcomes, Chen et al. [76] found that Guolin Walking Qigong reduces depressive symptoms over time, and similar results were found by Tsang et al. [78] with a BDJ intervention. Both these types of Qigong emphasize the active and physical components of Qigong.

From this review, we can see that both mindfulness and physical components of Qigong seem to help promote positive health outcomes. This is, perhaps, unsurprising, as mindfulness meditation [82] and regular physical exercise [83] have been shown to improve health in older adults. However, Qigong should not be conflated with either meditation and regular physical exercise or necessarily the sum of both parts, as Qigong involves distinct beliefs about energy meridians and blockages [2], which is not present in the other activities. Instead, future research should examine the unique quality of Qigong, including but not limited to, its cultural specificity and contextual belief system and whether this influences the magnitude of the health outcome.

\section{Intervention and control design}

Further, Qigong research has found some mixed preliminary findings regarding of the efficacy of Qigong compared to other forms of intervention or usual care. In comparing different kinds of exercise,
Qigong was found to be an effective practice to improve respiratory and physical function. Sakata et al. [2] examined two Qigong groups, where one of the groups additionally practiced some aerobic exercise. Given that there was no significant difference between groups in regard to physical function, the authors concluded that Qigong practice alone is an effective way to improve physical function. Chan et al. [75] and Xiao and Zhuang (2015) [61] both compared the physical function abilities of a Qigong practice group and a walking exercise group and found that the Qigong group showed significant and greater improvements in a six-minute walk test. In contrast, Oh et al. [72] found that when comparing the outcomes of Qigong exercise and exercise, there were no significant differences in quality of life outcomes, which may speak to how essential the meditative aspects of Qigong are in its practice. Notably, one study found few significant differences between Tai Chi and Qigong meditation in terms of gait performance [79], which indicates that there may not be large differences between these two mind-body forms. Among individuals with depressive symptoms, a study of 40 community-dwelling adults in Hong Kong by Chan et al. [75] found that Qigong was much more effective at reducing depressive mood than cognitive-behavioral therapy, a common psychotherapy technique, after only one month. The authors point to the cultural acceptability of Qigong among Chinese participants, while cognitive behavioral therapy is often used in western settings and may be less acceptable to non-western populations.

Many studies indicate that Qigong is more effective than usual care of wait-list control conditions at improving health outcomes. For example, Oh et al. [71] found significantly improved quality of life, fatigue, mood, and inflammation among a Qigong group compared to a control group after controlling for baseline health characteristics and sociodemographic information. In sum, Qigong interventions may produce similar health effects as other mind-body practices but also may be more efficacious at improving health than usual care. However, more research is needed to examine the differences between Qigong and other mind-body forms, as well as other forms of intervention to determine effectiveness, feasibility, and adherence for older adults.

\section{Length of practice}

There is some evidence that the length of practice can widely vary and still produce desirable health outcomes. A few studies indicate that 30 minutes of practice once is sufficient time for Qigong practice to produce psychological benefits, likely due to its similarities with other meditations which focus on positive affect $[47,56]$. In addition, the positive effects of Qigong can measurably last past a study's intervention period for at least 4 months. With an 8 -week intervention, Lynch et al. [81] found that participants who practiced at least 5 hours a week, as outlined in the protocol, sustained improved health regarding pain levels, sleep function, and overall health between the end of the eight-week intervention and the end of six month follow up. Further, the authors found that participants who practiced less than 3 hours a week during the eight-week intervention still experienced improved health, though not to the magnitude of individuals who practiced per protocol. This may be due to the efficacy of Qigong and/or the likelihood of older adults to maintain regular practice of Qigong. Indeed, there is some indication that Qigong is well suited for older adults due to its adaptability to functional ability and physical and meditative components [2,46]. Qigong's low intensity encourages continued exercise adherence [74]. Qigong appears to be effective regardless of practice length; however, more methodological rigorous research exploring the particular effects of the length of practice and effect of adherence is needed. 


\section{Limitations}

Despite these significant findings, there are some limitations to the current state of Qigong trial research among older adults. First, small sample size makes it difficult to ascertain effectiveness. Second, there issues regarding how Qigong interventions are conducted. Many articles do not reference the type of Qigong practiced which may influence the intended health effects. Further, there is no generic form of Qigong, which calls to question how closely the type of Qigong used in these research trials resembles traditional forms, whether the cultural component of Qigong influences researchers and participants, and whether Qigong is treated just as a low-intensity exercise.

Due to the lack of investigation in current literature, the role of culture or belief in Qigong practice remains plausible. In areas of biomedicine, cultural belief has been shown to impact compliance [84], which could also influence health outcomes. When investigating a practice or treatment such as Qigong which often explicitly incorporates non-biomedical beliefs about "energy," considering the influence of beliefs is necessary to examine which components may influence the outcome. Further, there is evidence that this concept of $Q i$, which does not have a biomedical analogue, is very important among Qigong practitioners [11]. Given a majority of the articles in this review do not include participants of East Asian descent and significant results are still found, it may be possible to conclude that Qigong has widespread appeal and the potential to be an effective intervention for older adults, independent of traditional cultural relevancy. However, there are unique experiences of Chinese and East Asian older adults [85-102], and it is necessary to thoroughly examine how culturally relevant practices like Qigong may specifically relate to their health.

\section{Future research directions}

In order to further understand the practice and effects of Qigong among older adults, there are multiple areas of research which should be addressed concerning study design, the complexities of Qigong, and the role of culture. It should be noted that CAM researchers have proposed a variety of directions for research pertaining to older adults which apply to Qigong research as well, including: understanding motivations for use or practice, safety concerns, longitudinal study design, larger sample size, including qualitative or ethnographic study design, and challenging the common health research approach of a biomedical framework [103]. Specific to research of older adults and Qigong, a variety of studies are needed to understand Qigong practice among Chinese older adults, since they are the most likely practitioners, as well as the specific health outcomes of Qigong practice among older adults. Longitudinal, population-based studies should be conducted in community-dwelling Chinese older adult communities to understand the current practice of and sociodemographic and health associations with Qigong. Existing studies of Chinese older adults have been able to understand the prevalence of health conditions and some health behaviors [89,104-108]; the next step should include information about culturally relevant exercise behaviors with additional qualitative interviews to understand their practice of Qigong.

In addition, research needs to be conducted which excludes middleage or young adults. A study design which includes adults of all ages is insufficient to ascertain how Qigong particularly affects health in older adult practitioners. Future studies should examine older adults alone given the different abilities to learn, practice, and adhere to exercise than younger adults. In addition, future research needs to evaluate the effectiveness of different forms of Qigong and other mind-body exercise, particularly Tai Chi, a similar and less meditative exercise to Qigong, in order to ascertain appropriateness of these exercises for older adults with different functional abilities.

Moreover, Qigong research should further investigate the possible mechanisms of Qigong health promotion. Many authors seem to indicate the relative success of Qigong is due to its equation with low intensity exercise or active physical therapy [58]. However, it is unknown if there is a particular belief or cultural influence in Qigong practice, adherence, or health effects. Clinical trial research should be standardized, as the current discrepancies in study design makes emerging trends difficult to define. This includes intervention and control designs, type of Qigong, consistent measurements, and other related covariates such as belief in CAM or Qi. There is some indication that other Chinese healing practices have undergone acculturation when adapted for Western populations [12]. However, to our knowledge, only one study [55] examined the correlates of CAM beliefs and Qigong-related health outcome. Future research should include an examination on whether cultural specificity impact CAM health outcomes, i.e. if Chinese practicing Qigong has greater impact on health versus non-Chinese practicing Qigong, and whether CAM beliefs, regardless of ethnic origin, impacts health outcomes. Relatedly, there are likely other physiological mechanisms which contribute to Qigong health outcomes which should be investigated further.

\section{Implications for practice and policy}

Despite the limitations of current Qigong research regarding older adults, this review has implications for health providers and policymakers. Recent CAM research of older adults has called for further integration of non-biomedical biomedical options for addressing certain health concerns [103]. We have found that Qigong may improve a variety of medical conditions and that it can be adapted for a variety of functional abilities, speaking towards its safety in practice and application. Health providers should provide information to older adults about Qigong as exercise, especially since there is some evidence that Qigong practice lowers medical costs and visits [10]. Relatedly, community leaders and policy makers should work towards making Qigong available to large groups of people. Research into exercise adherence among older adults has shown that psychosocial aspects of group exercise and exercise adaptability to a variety of functional abilities influences the likelihood of continued exercise and overall well-being [109-112]. In addition, there is evidence, though limited, that ethnic minority older adults prefer group exercise environments [113]. Integrating Qigong classes into community exercise offerings may be able to address these issues of maintaining exercise in advancing age, especially for minority adults who desire culturally-specific group exercise activities [114].

\section{Conclusion}

In conclusion, the existing body of research regarding Qigong and older adults indicate that Qigong may be an effective way of improving health outcomes, including overall quality of life, psychological distress, and pain. Future research of Qigong practice in older adult populations should specifically examine the role of relative age and health conditions in the feasibility and adaptability of Qigong exercise, health outcomes, and exercise adherence. Research methodology should rigorously evaluate Qigong versus other forms of mind-body exercise and whether cultural specificity and CAM beliefs affect health outcomes. Last, researchers, health providers, and community leaders should work in concert to investigate and improve the physical and psychosocial health and health behaviors of older adults through culturally appropriate and adaptable exercise like Qigong. 


\section{Acknowledgments}

We are grateful to Community Advisory Board members for their continued effort in this project. Particular thanks are extended to Bernie Wong, Vivian $\mathrm{Xu}$, and Yicklun Mo with the Chinese American Service League (CASL); Dr. David Lee with the Illinois College of Optometry; David Wu with the Pui Tak Center; Dr. Hong Liu with the Midwest Asian Health Association; Dr. Margaret Dolan with John H. Stroger Jr. Hospital; Mary Jane Welch with the Rush University Medical Center; Florence Lei with the CASL Pine Tree Council; Julia Wong with CASL Senior Housing; Dr. Jing Zhang with Asian Human Services; Marta Pereya with the Coalition of Limited English Speaking Elderly; and Mona El-Shamaa with the Asian Health Coalition.

\section{References}

1. Tsang HW, Cheung L, Lak DC (2002) Qigong as a psychosocial intervention for depressed elderly with chronic physical illnesses. Int J Geriatr Psychiatry 17: 11461154. [Crossref]

2. Kemp CA (2004) Qigong as a therapeutic intervention with older adults. J Holist Nurs 22: 351-373. [Crossref]

3. Jahnke R, Larkey L, Rogers C, Etnier J, Lin F (2010) A comprehensive review of health benefits of qigong and tai chi. Am J Health Promot 24: e1-1e25. [Crossref]

4. Otehode U (2009) The Creation and Reemergence of Qigong in China, in Making Religion, Making the State, D.L.W. Yoshiko A (Ed.), Stanford University Press 241-65.

5. Lee $\mathrm{S}$ (2000) Chinese hypnosis can cause qigong induced mental disorders. BMJ 320: 803. [Crossref]

6. McGee CT, Chow EPY (1994) Miracle Healing from China--Qigong. 1994: Medipress.

7. Birdee GS, Wayne PM, Davis RB, Phillips RS, Yeh GY (2009) T'ai chi and qigong for health: patterns of use in the United States. J Altern Complement Med 15: 969-973. [Crossref]

8. Ho TJ, Christiani DC, Ma TC, Jang TR, Lieng CH, et al. (2011) Effect of Qigong on quality of life: a cross-sectional population-based comparison study in Taiwan. $B M C$ Public Health 11: 546. [Crossref]

9. Lian WM, Gan GL, Pin CH, Wee S, Ye HC (1999) Correlates of leisure-time physical activity in an elderly population in Singapore. Am J Public Health 89: 1578-1580. [Crossref]

10. Yan X, Shen H, Loh C, Shao J, Yang Y, et al. (2013) A longitudinal study about the effect of practicing Yan Xin Qigong on medical care cost with medical claims data. Int J Econ Res 10: 391-403. [Crossref]

11. Jouper J, Hassmén P, Johansson M (2006) Qigong exercise with concentration predicts increased health. Am J Chin Med 34: 949-957. [Crossref]

12. Barnes LL (1998) The psychologizing of Chinese healing practices in the United States. Cult Med Psychiatry 22: 413-443. [Crossref]

13. Chao MT, Wade C, Kronenberg F, Kalmuss D, Cushman LF (2006) Women's reasons for complementary and alternative medicine use: Racial/ethnic differences. J Altern Complement Med 12: 719-720. [Crossref]

14. Siu JYM (2012) The Use of Qigong and Tai Chi as Complementary and Alternative Medicine (CAM) Among Chronically Ill Patients in Hong Kong. 2012: INTECH Open Access Publisher.

15. Bishop FL, Yardley L, Lewith GT (2007) A systematic review of beliefs involved in the use of complementary and alternative medicine. J Health Psychol 12: 851-867. [Crossref]

16. Dubbin LA, Chang JS, Shim JK (2013) Cultural health capital and the interactional dynamics of patient-centered care. Soc Sci Med 93: 113-120. [Crossref]

17. Ekman I, Swedberg K, Taft C, Lindseth A, Norberg A, et al. (2011) Person-centered care--ready for prime time. Eur J Cardiovasc Nurs 10: 248-251. [Crossref]

18. WHO traditional medicine strategy: 2014-2023, W.H. Organization, Editor. 2013, World Health Organization.

19. Roberti di Sarsina P (2007) The social demand for a medicine focused on the person: the contribution of CAM to healthcare and healthgenesis. Evid Based Complement Alternat Med 4: 45-51. [Crossref]
20. Arcury TA, Suerken CK, Grzywacz JG, Bell RA, Lang W, et al. (2006) Complementary and alternative medicine use among older adults: ethnic variation. Ethn Dis 16: 723 731. [Crossref]

21. Cheung CK, Wyman JF, Halcon LL (2007) Use of complementary and alternative therapies in community-dwelling older adults. J Altern Complement Med 13: 997-1006. [Crossref]

22. Oh B, Butow P, Mullan B, Hale A, Lee MS, et al. (2011) A critical review of the effect of medical Qigong on quality of life, immune function, and survival in cancer patients. Integr Cancer Ther 1534735411413268. [Crossref]

23. Xiong X, Wang P, Li S, Zhang Y, Li X (2015) Effect of Baduanjin exercise for hypertension: a systematic review and meta-analysis of randomized controlled trials. Maturitas 80: 370-378. [Crossref]

24. Wang CW, Chan CL, Ho RT, Tsang HW, Chan CH, et al. (2013) The effect of qigong on depressive and anxiety symptoms: a systematic review and meta-analysis of randomized controlled trials. Evid Based Complement Alternat Med 2013: 716094 [Crossref]

25. Cheng FK (2015) Effects of Baduanjin on mental health: a comprehensive review. $J$ Bodyw Mov Ther 19: 138-149. [Crossref]

26. Ng BH, Tsang HW (2009) Psychophysiological outcomes of health qigong for chronic conditions: a systematic review. Psychophysiology 46: 257-269. [Crossref]

27. Sawynok J, Lynch M (2014) Qigong and fibromyalgia: randomized controlled trials and beyond. Evid Based Complement Alternat Med 2014: 379715. [Crossref]

28. Lee MS, Chen KW, Sancier KM, Ernst E (2007) Qigong for cancer treatment: a systematic review of controlled clinical trials. Acta Oncol 46: 717-722. [Crossref]

29. Xiong X, Wang P, Li X, Zhang Y (2015) Qigong for hypertension: a systematic review. Medicine (Baltimore) 94: e352. [Crossref]

30. Lee MS, Chen KW, Choi TY, Ernst E (2009) Qigong for type 2 diabetes care: a systematic review. Complement Ther Med 17: 236-242. [Crossref]

31. Xin L, Miller YD, Brown WJ (2007) A qualitative review of the role of qigong in the management of diabetes. J Altern Complement Med 13: 427-433. [Crossref]

32. Rogers CE, Larkey LK, Keller C (2009) A review of clinical trials of tai chi and qigong in older adults. West $J$ Nurs Res 31: 245-279. [Crossref]

33. Lee MS, Chen KW, Ernst E (2010) Supportive cancer care with qigong, in Supportive Cancer Care with Chinese Medicine. 77-94.

34. Lauche R, Cramer H, Häuser W, Dobos G, Langhorst J (2013) A systematic review and meta-analysis of qigong for the fibromyalgia syndrome. Evid Based Complement Alternat Med 2013: 635182. [Crossref]

35. Liu X, Clark J, Siskind D, Williams GM, Byrne G, et al. (2015) A systematic review and meta-analysis of the effects of Qigong and Tai Chi for depressive symptoms. Complement Ther Med 23: 516-534. [Crossref]

36. Mei L, Chen Q, Ge L, Zheng G, Chen J (2012) Systematic review of Chinese traditional exercise Baduanjin modulating the blood lipid metabolism. Evid Based Complement Alternat Med 2012: 282131. [Crossref]

37. Barnes PM, et al. (2008) Complementary and alternative medicine use among adults and children: United States, 2007. 2008, US Department of Health and Human Services, Centers for Disease Control and Prevention, National Center for Health Statistics Hyattsville, MD.

38. Olano HA, Kachan D, Tannenbaum SL, Mehta A, Annane D, et al. (2015) Engagemen in Mindfulness Practices by US Adults: Sociodemographic Barriers. J Altern Complement Med 21: 100-102. [Crossref]

39. Fouladbakhsh JM, Stommel M (2010) Gender, symptom experience, and use of complementary and alternative medicine practices among cancer survivors in the US cancer population. Oncol Nurs Forum 37: E7-E15. [Crossref]

40. Komelski MF, Miyazaki Y, Blieszner R (2012) Comparing the health status of US Taijiquan and Qigong practitioners to a national survey sample across ages. J Altern Complement Med 18: 281-286. [Crossref]

41. Lee MS, Hong SS, Lim HJ, Kim HJ, Woo WH, et al. (2003) Retrospective survey on therapeutic efficacy of Qigong in Korea. Am J Chin Med 31: 809-815. [Crossref]

42. Leung Y, Singhal A (2004) An examination of the relationship between qigong meditation and personality. Social Behavior and Personality: an International Journal 32: $313-320$.

43. Chen K, Liu T (2004) Effects of qigong therapy on arthritis: A review and report of a 
pilot trial. Medical Paradigm 1: 36-48.

44. Chen KW, Hassett AL, Hou F, Staller J, Lichtbroun AS (2006) A pilot study of external qigong therapy for patients with fibromyalgia. J Altern Complement Med 12: 851-856. [Crossref]

45. Cohen L, Chen Z, Arun B, Shao Z, Dryden M, et al. (2010) External qigong therapy for women with breast cancer prior to surgery. Integr Cancer Ther 9: 348-353. [Crossref]

46. Sakata T, Li Q, Tanaka M, Tajima F (2008) Positive effects of a qigong and aerobic exercise program on physical health in elderly Japanese women: an exploratory study. Environ Health Prev Med 13: 162-168. [Crossref]

47. Johansson M, Hassmén P (2013) Affective responses to qigong: a pilot study of regular practitioners. J Bodyw Mov Ther 17: 177-184. [Crossref]

48. Sawynok J, Lynch M, Marcon D (2013) Extension trial of qigong for fibromyalgia: a quantitative and qualitative study. Evid Based Complement Alternat Med 2013: 726062. [Crossref]

49. Liu X, Miller YD, Burton NW, Brown WJ (2010) A preliminary study of the effects of Tai Chi and Qigong medical exercise on indicators of metabolic syndrome, glycaemic control, health-related quality of life, and psychological health in adults with elevated blood glucose. Br J Sports Med 44: 704-709. [Crossref]

50. Liu W, Zahner L, Wang Y (2012) Qigong Exercise in Patients With Fibromyalgia Two Cases. Journal of Evidence-Based Complementary \& Alternative Medicine 18: 80-85.

51. Kuan S, Chen K, Wang C (2012) Effectiveness of qigong in promoting the health of wheelchair-bound older adults in long-term care facilities. Biol Res Nurs 14: 139-146. [Crossref]

52. Overcash J, Will KM, Lipetz DW (2013) The benefits of medical qigong in patients with cancer: a descriptive pilot study. Clin J Oncol Nurs 17: 654-658. [Crossref]

53. Wu X, Li L, Ding P (2014) Effect of Baduanjin on 62 patients with Coronary heart disease and depression. World Chinese Medicine 9: 39-40.

54. Johansson M, Hassmén P (2008) Acute psychological responses to Qigong exercise of varying durations. Am J Chin Med 36: 449-458. [Crossref]

55. Chen KW, Perlman A, Liao JG, Lam A, Staller J, et al. (2008) Effects of external qigong therapy on osteoarthritis of the knee. A randomized controlled trial. Clin Rheumatol 27: 1497-1505. [Crossref]

56. Johansson M, Hassmén P, Jouper J (2011) Acute effects of Qigong exercise on mood and anxiety. International Journal of Stress Management 15: 199-207.

57. Cai, J, et al. (2015) Observation of 30 cases of Baduanjin combined with routine therapy in the recovery of patients with nerve root type cervical spondylosis. Guiding Journal of Traditional Chinese Medicine and Pharmacy 21: 98-99.

58. Schmitz-Hübsch T, Pyfer D, Kielwein K, Fimmers R, Klockgether T, et al. (2006) Qigong exercise for the symptoms of Parkinson's disease: a randomized, controlled pilot study. Mov Disord 21: 543-548. [Crossref]

59. Wang JY, Guo H, Tang L, Meng J, Hu Ly (2014) Case-control study on regular Ba Duan Jin practice for patients with chronic neck pain. International Journal of Nursing Sciences 1: 360-366.

60. Wang S, Zhu HX, Zhang Y, Zeng YG, Wang AL (2007) The effects of New Baduanjin on Middle/Old aged people's Life Quality. Journal of Beijing Sport University 20: 203-205.

61. Xiao CM, Zhuang YC (2015) Efficacy of Liuzijue Qigong in Individuals with Chronic Obstructive Pulmonary Disease in Remission. J Am Geriatr Soc 63: 1420-1425. [Crossref]

62. Xiao CM, Zhuang YC (2015) Effect of health Baduanjin Qigong for mild to moderate Parkinson's disease. Geriatr Gerontol Int. [Crossref]

63. Yang Y, Verkuilen JV, Rosengren KS, Grubisich SA, Reed MR, et al. (2007) Effect of combined Taiji and Qigong training on balance mechanisms: a randomized controlled trial of older adults. Med Sci Monit 13: 339-348.

64. Liu X, et al., Study on influence of eight-sectioned exercise on fall efficacy in community elderly. Chinese Nursing Research 29: 90-93.

65. Wenneberg S, Gunnarsson LG, Ahlström G (2004) Using a novel exercise programme for patients with muscular dystrophy. Part II: a quantitative study. Disabil Rehabil 26: 595-602. [Crossref]

66. Oh B, Butow PN, Mullan BA, Clarke SJ, Beale PJ, et al. (2012) Effect of medical Qigong on cognitive function, quality of life, and a biomarker of inflammation in cancer patients: a randomized controlled trial. Supportive Care Cancer 20: 1235-1242. [Crossref]
67. Wei Q, Wu Y (2014) Clinical study of Baduanjin on health status in patients with type 2 diabetes. Journal of Liaoning University of Traditional Chinese Medicine 16: 103-105.

68. Lansinger B, Larsson E, Persson LC, Carlsson JY (2007) Qigong and exercise therapy in patients with long-term neck pain: a prospective randomized trial. Spine (Phila Pa 1976) 32: 2415-2422. [Crossref]

69. von Trott P, Wiedemann AM, Lüdtke R, Reishauer A, Willich SN, et al. (2009) Qigong and exercise therapy for elderly patients with chronic neck pain (QIBANE): a randomized controlled study. J Pain 10: 501-508. [Crossref]

70. Chan AW, Lee A, Suen LK, Tam WW (2011) Tai chi Qigong improves lung functions and activity tolerance in COPD clients: a single blind, randomized controlled trial Complement Ther Med 2011. 19: 3-11. [Crossref]

71. Oh B, Butow P, Mullan B, Clarke S, Beale P, et al. (2010) Impact of medical Qigong on quality of life, fatigue, mood and inflammation in cancer patients: a randomized controlled trial. Ann Oncol 21: 608-614. [Crossref]

72. Oh B, Butow P, Boyle F, Beale P, Costa DSJ, et al. (2014) Effects of Qigong on Quality of Life, Fatigue, Stress, Neuropathy, and Sexual Function in Women with Metastatic Breast Cancer: A Feasibility Study. Int J Phys Med Rehabil 2: 2.

73. Oh B, Butow P, Mullan B, Clarke S (2008) Medical Qigong for cancer patients: pilot study of impact on quality of life, side effects of treatment and inflammation. Am J Chin Med 36: 459-472. [Crossref]

74. Jouper J, Hassmén P (2009) Exercise intention, age and stress predict increased qigong exercise adherence. J Bodyw Mov Ther 13: 205-211. [Crossref]

75. Chan AS, Cheung MC, Tsui WJ, Sze SL, Shi D (2011) Dejian mind-body intervention on depressive mood of community-dwelling adults: a randomized controlled trial. Evid Based Complement Alternat Med 2011:473961. [Crossref]

76. Chen Z, Meng Z, Milbury K, Bei W, Zhang Y, et al. (2013) Qigong improves quality of life in women undergoing radiotherapy for breast cancer: results of a randomized controlled trial. Cancer 119: 1690-1698. [Crossref]

77. Hsieh CJ, Chang C, Tsai G, Wu HF (2015) Empirical study of the influence of a Laughing Qigong Program on long-term care residents. Geriatr Gerontol Int 15: 165 173. [Crossref]

78. Tsang HW, Tsang WW, Jones AY, Fung KM, Chan AH, et al. (2013) Psycho-physical and neurophysiological effects of qigong on depressed elders with chronic illness. Aging Ment Health 17: 336-348. [Crossref]

79. Amano S, Nocera JR, Vallabhajosula S, Juncos JL, Gregor RJ, et al. (2013) The effect of Tai Chi exercise on gait initiation and gait performance in persons with Parkinson's disease. Parkinsonism Relat Disord 19: 955-960. [Crossref]

80. Bijur PE, Silver W, Gallagher EJ (2001) Reliability of the visual analog scale for measurement of acute pain. Acad Emerg Med 8: 1153-1157. [Crossref]

81. Lynch M, Sawynok J, Hiew C, Marcon D (2012) A randomized controlled trial of qigong for fibromyalgia. Arthritis Res Ther 14: R178. [Crossref]

82. Chiesa A, Serretti A (2011) Mindfulness-based interventions for chronic pain: a systematic review of the evidence. J Altern Complement Med 17: 83-93. [Crossref]

83. Suzuki T, Kim H, Yoshida H, Ishizaki T (2004) Randomized controlled trial of exercise intervention for the prevention of falls in community-dwelling elderly Japanese women. J Bone Miner Metab 22: 602-611. [Crossref]

84. Ross S, Walker A, MacLeod MJ (2004) Patient compliance in hypertension: role of illness perceptions and treatment beliefs. J Hum Hypertens 18: 607-613. [Crossref]

85. Dong X, Bergren SM1, Chang ES1 (2015) Levels of Acculturation of Chinese Older Adults in the Greater Chicago Area - The Population Study of Chinese Elderly in Chicago. J Am Geriatr Soc 63: 1931-1937. [Crossref]

86. Dong X, Chang ES, Bergren S (2014) The Burden of Grandparenting among Chinese older adults in the Greater Chicago area-The PINE Study. AIMS Medical Science 1: 125-140.

87. Dong X, Chang ES, Simon MA (2014) Physical Function Assessment in a Community Dwelling Population of US Chinese Older Adults. J Gerontol A Biol Sci Med Sci 69 S31-S38. [Crossref]

88. Dong X, Chen R, Li C, Simon MA (2014) Understanding Depressive Symptoms Among Community-Dwelling Chinese Older Adults in the Greater Chicago Area. $J$ Aging Health 26: 1155-1171. [Crossref]

89. Dong X, Chen R, Simon MA (2014) The Prevalence of Medical Conditions Among US Chinese Community-Dwelling Older Adults. J Gerontol A Biol Sci Med Sci 69: S15-S22. [Crossref] 
90. Dong X, Chen R, Simon MA (2014) Experience of Discrimination among US Chinese Older Adults. J Gerontol A Biol Sci Med Sci 69: S76-81. [Crossref]

91. Dong X, Chen R, Simon MA (2014) Anxiety among Community-Dwelling US Chinese Older Adults. J Gerontol A Biol Sci Med Sci 69: p. S61-S67. [Crossref]

92. Dong X, Chen R, Wong E, Simon MA (2014) Suicidal ideation in an older U.S. Chinese population. J Aging Health 26: 1189-1208. [Crossref]

93. Dong X, Li Y, Simon MA (2014) Social engagement among U.S. Chinese older adults-findings from the PINE Study. J Gerontol A Biol Sci Med Sci 69 Suppl 2: S82-89. [Crossref]

94. Dong X, Zhang M, Simon M (2014) The Prevalence of Cardiopulmonary Symptoms Among Chinese Older Adults in the Greater Chicago Area. J Gerontol A Biol Sci Med Sci 69: S39-S45. [Crossref]

95. Dong X, Zhang M, Simon MA (2014) The expectation and perceived receipt of filial piety among Chinese older adults in the Greater Chicago area. J Aging Health 26 : 1225-1247. [Crossref]

96. Dong X, Zhang M, Simon MA (2014) Self-mastery among Chinese Older Adults in the Greater Chicago Area. AIMS Medical Science 1: 57-72.

97. Simon MA, Chang ES, Zhang M, Ruan J, Dong X (2014) The prevalence of loneliness among U.S. Chinese older adults. J Aging Health 26: 1172-1188. [Crossref]

98. Simon MA, Chen R, Chang ES, Dong X (2014) The Association Between Filia Piety and Suicidal Ideation: Findings From a Community-Dwelling Chinese Aging Population. J Gerontol A Biol Sci Med Sci 69: S90-S97. [Crossref]

99. Simon MA, Li Y, Dong X (2014) Preventive Care Service Usage Among Chinese Older Adults in the Greater Chicago Area. J Gerontol A Biol Sci Med Sci 69: p. S7-S14.

100. Simon MA, Li Y, Dong X (2014) Levels of Health Literacy in a CommunityDwelling Population of Chinese Older Adults. J Gerontol A Biol Sci Med Sci 69: S54-S60. [Crossref]

101. Simon MA, Zhang M, Dong X (2014) Trust in Physicians Among US Chinese Older Adults. J Gerontol A Biol Sci Med Sci 69: S46-S53. [Crossref]

102. Zhang M, Simon MA, Dong X (2014) The Prevalence of Perceived Stress among US Chinese Older Adults. AIMS Medical Science 1: 40-56.

103. Willison KD, Andrews GJ (2004) Complementary medicine and older people: past research and future directions. Complement Ther Nurs Midwifery 10: 80-91. [Crossref]

104. Dong X, Bergren SM, Chang E (2015) Traditional Chinese Medicine Use and Health in Community-Dwelling Chinese-American Older Adults in Chicago. $J$ Am Geriatr Soc 63: 2588-2595. [Crossref]

105. Dong X, Chang ES, Wong E, Wong B, Skarupski KA, et al. (2011) Assessing the health needs of Chinese older adults: Findings from a community-based participatory research study in Chicago's Chinatown. J Aging Res 2010: 124246.

106. Dong X, Chang ES, Bergren S (2014) The Prevalence of Musculoskeletal Symptoms among Chinese older adults in the Greater Chicago area-Findings from the PINE study. AIMS Medical Science 1: 87-102.

107. Dong X, et al. (2011) Community-Based Participatory Research Approach to Assess the Health Needs of Chinese Older Adults, in Gerontological Society of America: 64th Annual Scientific Meeting. The Gerontologist: Boston, MA.

108. Dong X, Wong E, Simon MA (2014) Study design and implementation of the PINE study. J Aging Health 26: 1085-1099. [Crossref]

109. Deforche B, De Bourdeaudhuij I (2000) Differences in psychosocial determinants of physical activity in older adults participating in organised versus non-organised activities. J Sports Med Phys Fitness 40: 362. [Crossref]

110. King AC (2001) Interventions to promote physical activity by older adults. $J$ Gerontol A Biol Sci Med Sci 56 Spec No 2: 36-46. [Crossref]

111. McAuley E, Blissmer B, Marquez DX, Jerome GJ, Kramer AF, et al. (2000) Socia relations, physical activity, and well-being in older adults. Prev Med 31: 608-617. [Crossref]

112. Brawley LR, Rejeski WJ, King AC (2003) Promoting physical activity for older adults: the challenges for changing behavior. Am J Prev Med 25: 172-183. [Crossref]

113. Chiang KC, Seman L, Belza B, Tsai JH (2008) "It is our exercise family": experiences of ethnic older adults in a group-based exercise program. Prev Chronic Dis 5: A05. [Crossref]

114. Belza B, Walwick J, Shiu-Thornton S, Schwartz S, Taylor M, et al. (2004) Older adult perspectives on physical activity and exercise: voices from multiple cultures. Prev Chronic Dis 1: A09. [Crossref]

115. Wei R, et al. (2012) The effects of Baduanjin on the improvement of body function of patients with Ankylosing Spondylitis. Journal of North Pharmacy 9: 67-68.

116. Yang KH, Kim YH, Lee MS (2005) Efficacy of Qi-therapy (external Qigong) for elderly people with chronic pain. Int J Neurosci 115: 949-963. [Crossref]

117. Lee MS, Yang KH, Huh HJ, Kim HW, Ryu H, et al. (2001) Qi therapy as an intervention to reduce chronic pain and to enhance mood in elderly subjects: a pilot study. Am J Chin Med 29: 237-245. [Crossref]

118. Wang B, Ma S, Hu Y (2015) Effects of Yijinjing on the prevention of skeletal muscle weakness for senior adults. Chinese Journal of Gerontology 35 .

119. Li R, Jin L, Hong P, He ZH, Huang CY, et al. (2014) The effect of baduanjin on promoting the physical fitness and health of adults. Evid Based Complement Alternat Med 2014: 784059. [Crossref]

120. Maddali Bongi S, Del Rosso A, Di Felice C, Calà M, Giambalvo Dal Ben G (2011) Resseguier method and Qi Gong sequentially integrated in patients with fibromyalgia syndrome. Clin Exp Rheumatol 30: 51-58. [Crossref]

121. Pippa L, Manzoli L, Corti I, Congedo G, Romanazzi L, et al. (2007) Functional capacity after traditional Chinese medicine (qi gong) training in patients with chronic atrial fibrillation: a randomized controlled trial. Prev Cardiol 10: 22-25. [Crossref]

122. Burini D, Farabollini B, Iacucci S, Rimatori C, Riccardi G, et al. (2006) A randomised controlled cross-over trial of aerobic training versus Qigong in advanced Parkinson's disease. Eura Medicophys 42: 231-238. [Crossref]

123. Stenlund T, Lindström B, Granlund M, Burell G (2005) Cardiac rehabilitation for the elderly: Qi Gong and group discussions. Eur J Cardiovasc Prev Rehabil 12: 5-11. [Crossref]

124. Astin JA, Berman BM, Bausell B, Lee WL, Hochberg M, et al. (2003) The efficacy of mindfulness meditation plus Qigong movement therapy in the treatment of fibromyalgia: a randomized controlled trial. J Rheumatol 30: 2257-2262. [Crossref]

125. Tsang HW, Lee JL, Au DW, Wong KK, Lai KW (2013) Developing and testing the effectiveness of a novel health qigong for frail elders in Hong Kong: a preliminary study. Evid Based Complement Alternat Med 2013: 827392. [Crossref]

126. Chow YWY, Dorcas A, Siu AMH (2012) The effects of qigong on reducing stress and anxiety and enhancing body-mind well-being. Mindfulness 3: 51-59.

127. Stenlund T, Birgander LS, Lindahl B, Nilsson L, Ahlgren C (2009) Effects of Qigong in patients with burnout: a randomized controlled trial. J Rehabil Med 41: 761-767. [Crossref]

128. Griffith JM, Hasley JP, Liu H, Severn DG, Conner LH, et al. (2008) Qigong stress reduction in hospital staff. $J$ Altern Complement Med 14: 939-945. [Crossref]

129. Haak T, Scott B (2008) The effect of Qigong on fibromyalgia (FMS): a controlled randomized study. Disabil Rehabil 30: 625-633. [Crossref]

130. Tsang HW, Fung KM, Chan AS, Lee G, Chan F (2006) Effect of a qigong exercise programme on elderly with depression. Int J Geriatr Psychiatry 21: 890-897. [Crossref]

131. Tsang HW, Mok CK, Au Yeung YT, Chan SY (2003) The effect of Qigong on general and psychosocial health of elderly with chronic physical illnesses: a randomized clinical trial. Int J Geriatr Psychiatry 18: 441-449.

132. Lin XL, Chen JW, Zhang GQ, Zhao JY, Tang C (2012) Effects of Eight Sections Brocade (Ba Duan Jin) on QUality of Life for patients after Cornary Artery Bypass Grafting. Journal of Nursing 19.

133. Liao Y, Lin Y, Zhang C, Xue XL, Mao QX, et al. (2015) Intervention Effect of Baduanjin Exercise on the Fatigue State in People with Fatigue-Predominant Subhealth: A Cohort Study. J Altern Complement Med 21: 554-562. [Crossref]

134. Larkey LK, Roe DJ, Weihs KL, Jahnke R, Lopez AM, et al. (2015) Randomized controlled trial of Qigong/Tai Chi Easy on cancer-related fatigue in breast cancer survivors. Ann Behav Med 49: 165-176. [Crossref]

135. Li L, Wang N (2014) Research Progress of Baduanjin in the treatment of insomnia patients with type 2 diabetes. Nei Mongol Journal of Traditional Chinese Medicine 27: $86-87$ 
136. Chan AS, Wong QY, Sze SL, Kwong PP, Han YM, et al. (2012) A Chinese Chan-based mind-body intervention improves sleep on patients with depression: a randomized controlled trial. The Scientific World Journal, 2012. [Crossref]
137. Chen MC, Liu HE, Huang HY, Chiou AF (2012) The effect of a simple traditional exercise programme (Baduanjin exercise) on sleep quality of older adults: a randomized controlled trial. Int J Nurs Stud 49: 265-273. [Crossref]

Copyright: $@ 2016$ Dong X. This is an open-access article distributed under the terms of the Creative Commons Attribution License, which permits unrestricted use, distribution, and reproduction in any medium, provided the original author and source are credited. 